\title{
High Temperature Superconductors \\ Through the Van Hove Singularity
}

By Keryn Anne Williams

A thesis submitted to

The Victoria University of Wellington

In fulfilment of the degree of

Master of Science

In Physics

Victoria University of Wellington

2009 


\begin{abstract}
The antibonding VHS of the high temperature superconductor Bi-2212 appears in the extreme overdoped regime, a part of the cuprate phase diagram little studied to date. Observation of this VHS motivated taking a fresh look at the cuprates using fundamentals of electronics as the foundation for understanding the physics involved in the superconductivity of these materials. In the study of the high temperature superconductors it appears important questions have been overlooked, notably the possible contribution of the gapped state and whether these materials are better considered as doped semiconductors rather than as 'poor' metals. We also find the question of the contribution of oxygen, a substance with a strong magnetic signature, to data of the oxygen-doped cuprates has been neglected. Comparison with non-oxygen doping is supportive of the view the oxygen dopant contributes noticeably to magnetic data.
\end{abstract}

Through magnetic susceptibility measurements the antibonding VHS location, predicted by use of Fermi liquid theory, is well confirmed in polycrystals of the lead-doped cuprate $\mathrm{Bi}-2212$. It was found that the peak in the DOS at the VHS produces no corresponding local peak in the critical temperature versus doping. Instead, the VHS appears associated with the disappearance of the superconductivity, rather than with the maximum critical temperature. 
We find the metal-insulator transition plays an important role. There are two of these in the cuprates, a horizontal doping dependent one and a vertical temperature dependent one. They affect each other. Noting the consequences of doping an insulator until a metallic state is reached enables a connection to be made between doping and pressure.

Three requirements are identified for superconductivity to occur:

1. screening

2. pairing

3. charge mobility

Each requirement may be separately satisfied in a manner whereby each can vary differently as a function of the same variable.

The superconductivity of the cuprates is found to arise out of an underlying non-metallic state. As such, BCS theory, being formulated to explain superconductivity arising from metallic conduction, cannot be directly applicable. However, although HTS materials are a rich repository of both novel and familiar solid state physics, evidence does not appear to support the notion that superconductivity in the cuprates is caused by "exotic" physics.

We also find cause for optimism regarding the development of new or improved superconducting materials. 


\section{Acknowledgements}

Many thanks to my supervisor Dr. Jeff Tallon for the interesting discussions and being willing to accept an unconventional student. Again, special thanks to Dr. Grant Williams of Industrial Research Limited for the open door.

Thanks are due also to Prof. Malcolm McMahon, University of Edinburgh, for taking the time to send information on bulk oxygen characteristics to a stranger on the other side of the world.

To Dr. James Storey, Cavendish Laboratory, many thanks for provision of information and continuing discussion after leaving for the other side of the world.

I am indebted to the HTS Platform of Industrial Research Ltd., Wellington, for having me. It has been a really great experience. And the support of the Science Faculty office is greatly appreciated.

Also, I am very thankful and privileged for all the help and permissions so cheerfully given by both publishers and authors. Specially acknowledgement to Professor John Cooper for even sending me the raw data behind a paper, that I may create my own graph. It has been a pleasure to have the need to request copyright permissions. Any error resulting from the display of other people's work is all mine. 
In accordance with the reproduction agreement with the American Physical Society (APS) with regard to the figures reproduced from publications of theirs (Phys. Rev. B and Phys. Rev. Lett.), the following statement is proclaimed: “ Readers may view, browse, and/or download material for temporary copying purposes only, provided these uses are for noncommercial personal purposes. Except as provided by law, this material may not be further reproduced, distributed, transmitted, modified, adapted, performed, displayed, published, or sold in whole or part, without prioir written permission from the American Physical Society." 


\section{Contents}

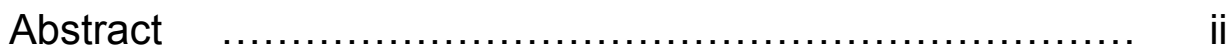

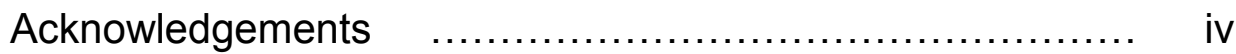

List of figures $\quad$................................................. viii

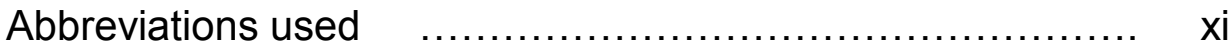

1. Motivation $\quad$.................................................. 1

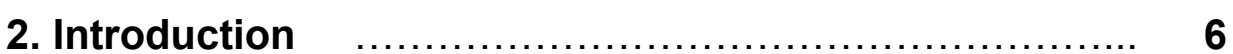

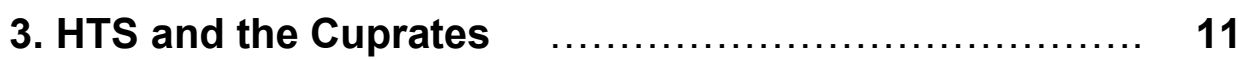

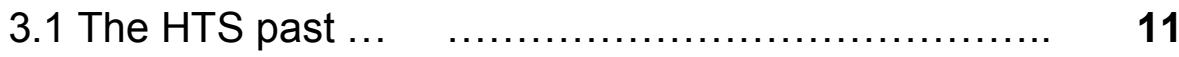

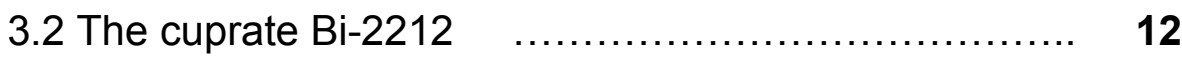

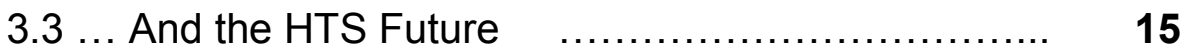

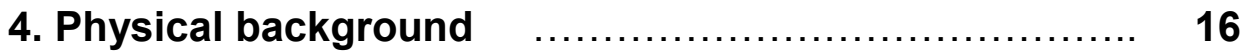

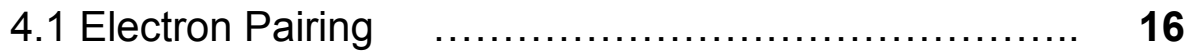

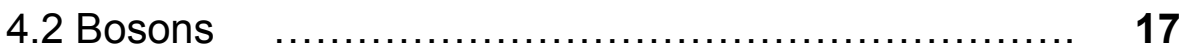

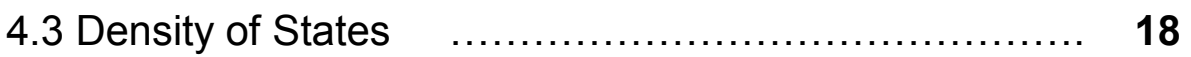

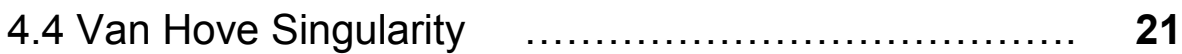

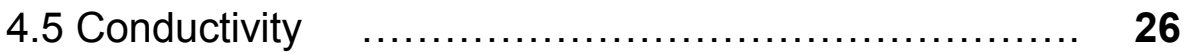

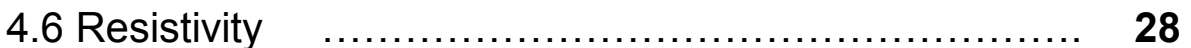

4.7 Degenerate Semiconductors (i) $\ldots \ldots \ldots \ldots \ldots \ldots \ldots \ldots . . . \ldots 29$

4.8 The Metal-Insulator Transition $\quad$........................ 30

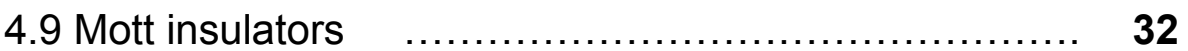

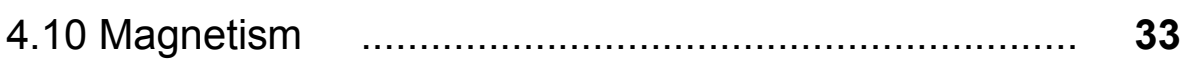

4.10.1 Diamagnetism …….............................. 33

4.10.2 Paramagnetism ．........................................ 34

4.10.3 Ferromagnetism ................................... 34

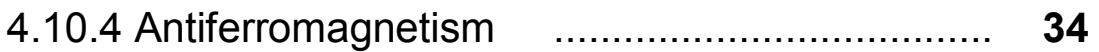

4.10.5 Ferrimagnetism $\quad$...................................... $\quad 35$ 


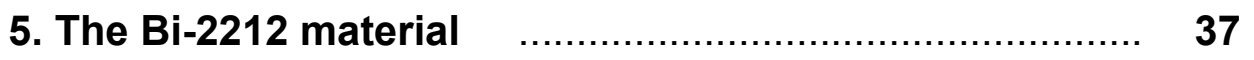

5.1 The samples used in this experiment $\quad \ldots \ldots \ldots \ldots \ldots \ldots . . . \ldots . . .37$

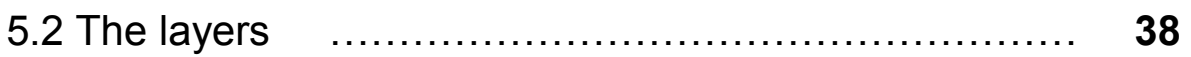

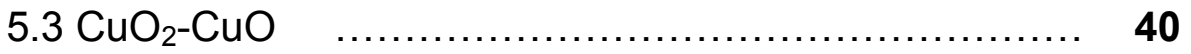

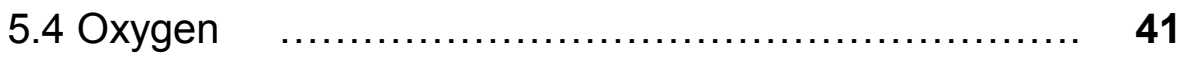

6. Experiment ……........................................... 51

6.1 Data acquisition $\quad$................................................. 51

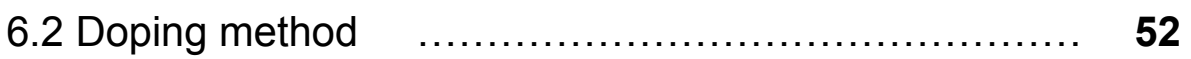

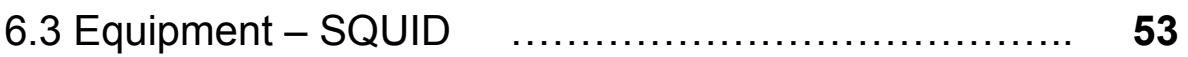

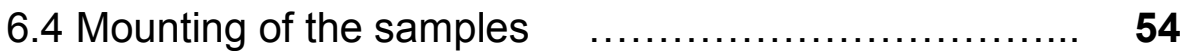

6.5 Main Polycrystal $\quad$.................................................. 58

6.6 Single Crystal .................................................. 71

6.7 Control Polycrystal $\quad$................................................. 78

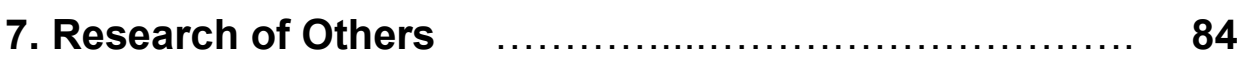

7.1 Normal State Resistivity under the

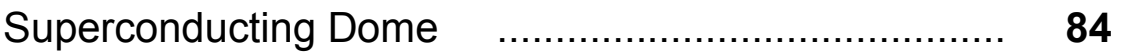

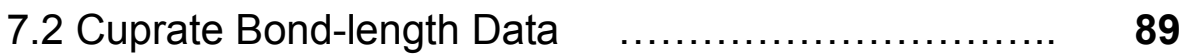

7.3 Superconductivity as a function of normal

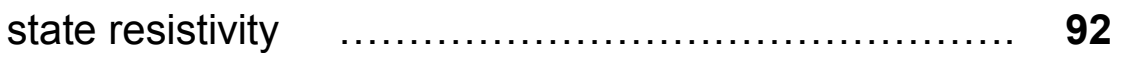

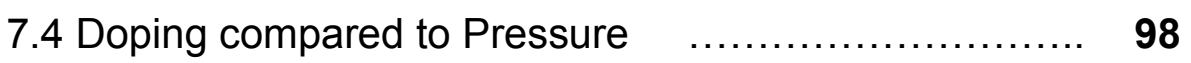

7.4.1 Magnetic Breakdown as a function of

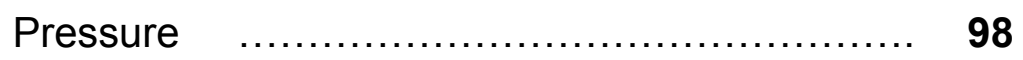

7.4.2 Conductivity as a function of Magnetic

Breakdown ................................. 99

7.4.3 Conductivity as a function of Pressure $\quad \ldots \ldots \ldots . .101$

7.4.4 Conductivity as a function of Doping $\ldots . . . \ldots \ldots . . . .101$

7.4.5 Magnetic Breakdown as a function of

Doping ................................................ 101

7.4.6 Equivalence of Doping and Pressure $\quad \ldots . . . \ldots \ldots . . .102$ 
7.5 Degenerate semiconductors (II)

7.6 Optically Transparent Semiconductors $\quad$............... 106

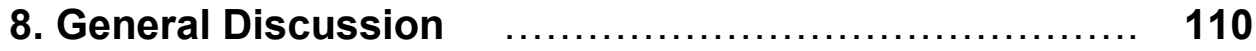

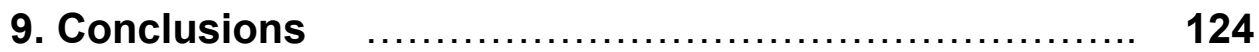

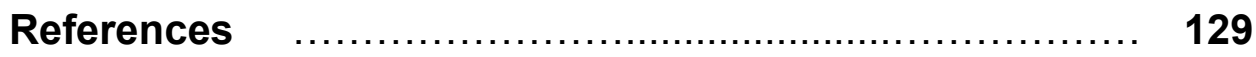

\section{List of Figures}

$\begin{array}{clc}\begin{array}{c}\text { Figure } \\ 1\end{array} & \begin{array}{l}\text { Excerpt from the preliminary view of the VHS } \\ \text { in lead-doped Bi-2212 }\end{array} & 3 \\ 2 & \begin{array}{l}\text { The same view of the VHS with the 'anomaly' } \\ \text { included }\end{array} & 4 \\ 3 & \begin{array}{l}\text { A. The perovskite tetrahedra } \\ \text { B. The unit cell of Bi-2212 }\end{array} & 13 \\ 4 & \begin{array}{l}\text { Generic phase diagram for hole-doped } \\ \text { cuprates }\end{array} & 14 \\ 5 & \begin{array}{l}\text { The Bi-2212 dispersion } \\ 6\end{array} & 19 \\ \text { Density of states depicted in 1D } & 20\end{array}$

viii 
8 Theoretical prediction of the magnetic

susceptibility as a function of temperature

9 Schematic of magnetic susceptibility of cupric oxide, $\mathrm{CuO}$

10 Phase diagram for the $\mathrm{O}_{2} /$ graphite system

11 Susceptibility of cooled oxygen in constant volume

12 Specific heat of physisorbed oxygen

13 Single crystal in Teflon ${ }^{(}$, gelatine and straw

$14 \operatorname{Teflon}^{\circledR}$ magnetic susceptibility

15 Plastic straw bulk magnetic susceptibility

$16 \quad(\mathrm{Bi}, \mathrm{Pb})-2212$ polycrystal examples of magnetic susceptibility versus temperature

$17 \quad(\mathrm{Bi}, \mathrm{Pb})-2212$ polycrystal magnetic susceptibility versus $T_{c}$

$18(\mathrm{Bi}, \mathrm{Pb})-2212$ polycrystal VHS at low temperature isotherms

19 Theoretical isotherms in the range $90 \mathrm{~K}-115 \mathrm{~K}$

20 Theoretical isotherms in the range 150K-310K

21 Effects of reannealing and of measuring ZFC or FC

22 Our polycrystal data for isotherms $200 \mathrm{~K}$ and $300 \mathrm{~K}$

23 Triscone et al. polycrystal data for isotherms $200 \mathrm{~K}$ and $300 \mathrm{~K}$

24 The VHS in the magnetic susceptibility of the single layer cuprate LSCO

25 Single crystal raw data of normal state susceptibilities

26 Progression of single crystal anneals at low temperature 
27 Single crystal susceptibility as a function of

anneal conditions

$28 \quad(\mathrm{Bi}, \mathrm{Pb})-2212$ Control polycrystal raw data

29 Control polycrystal susceptibility as a function

of anneal conditions

30 Companion polycrystal VHS and anomaly

31 VHS locations

32 Resistivity data of Boebinger et al. for the $a b$

plane of LSCO

33 Enlargement of the Boebinger ab data for higher doping

34 Resistivity data of Boebinger et al. for the caxis direction of LSCO

35 Doping dependent MIT of $\mathrm{Ca}_{1-\mathrm{x}} \mathrm{Li}_{x} \mathrm{Pd}_{3} \mathrm{O}_{4} \quad \mathbf{8 8}$

36 Relation of $\mathrm{T}_{\mathrm{c}}$ to $\mathrm{Cu}-\mathrm{O}$ bond length for various $\quad 90$ cuprates

$37 \quad T_{C}$ vs bond length for six cuprate families

38 Cuprates phase diagram adapted from S. Uchida

39 Examples of $T_{c}$ versus pressure curves for elements

40 Superconductivity vs room temperature conductivity for the elements under both ambient and applied pressure 


\begin{tabular}{|c|c|}
\hline $2 \mathrm{D}$ & 2-dimension(al) \\
\hline ac & alternate current \\
\hline ARPES & Angle-Resolved Photo Emission Spectrography \\
\hline Bi-2212 & BSCCO with $\mathrm{n}=2\left(\mathrm{Bi}_{2} \mathrm{Sr}_{2} \mathrm{CaCu}_{2} \mathrm{O}_{8+\delta}\right)$ \\
\hline$(\mathrm{Bi}, \mathrm{Pb})-2212$ & $\mathrm{Bi}-2212$ with some of the $\mathrm{Bi}^{3+}$ replaced with $\mathrm{Pb}^{2+}$ \\
\hline BSCCO & Bismuth-Strontium-Calcium-Copper-Oxide cuprate \\
\hline CPL-1 & Coordination Polymer (that forms 1D nanospace) \\
\hline dc & direct current \\
\hline DOS & Density of States \\
\hline FC & Field Cooled \\
\hline HIP & Hot Isostatic Pressing \\
\hline HTS & High Temperature Superconductor/Superconductivity \\
\hline LSCO & the cuprate $\mathrm{La}_{2-x} \mathrm{Sr}_{x} \mathrm{CuO}_{4}$ \\
\hline MIT & Metal-Insulator Transition \\
\hline $\mathrm{p}$ & doping level of the $\mathrm{Bi}-2212$ in holes per copper atom \\
\hline QCP & Quantum Critical Point \\
\hline SQUID & Superconducting QUantum Interference Device \\
\hline $\mathrm{T}_{\mathrm{c}}$ & Critical Temperature for Superconductivity \\
\hline TCO & Transparent Conducting (or Conductive) Oxide \\
\hline TOS & Transparent Oxide Semiconductors \\
\hline VHS & Van Hove Singularity \\
\hline YBCO & the cuprate $\mathrm{YB}_{2} \mathrm{C}_{3} \mathrm{O}_{7}$ \\
\hline ZFC & Zero Field Cooled \\
\hline$\chi$ & Magnetic susceptibility \\
\hline$\rho$ & Electrical resistivity, unless otherwise stated. \\
\hline
\end{tabular}




\section{Motivation}

Superconductors are characterised by the opening of an energy gap at the critical temperature, $T_{c}$. Both $T_{c}$ and the magnitude of the energy gap are governed, in conventional theory, by the density of states (DOS) and the pairing interaction. The DOS therefore plays an important role in superconductivity and possibly a crucial role in high temperature superconductors. These are layered 2D electronic materials in which there occurs a van Hove singularity where the DOS diverges.

A Van Hove singularity is a critical point in the density of states. In 2D systems this takes the form of a peak in the DOS within a band and is associated with a change between hole and electron majority charge carrier. Theoretically, the presence of a VHS in a material results from the effect the periodic structure of a crystal has on the frequency distribution of vibrations [1]. Van Hove applied his original analysis to phonons but the effect is also valid for the frequency (and hence energy) distribution of the electrons within a material. With the discovery of the cuprates interest intensified in the physical consequences of the 2D VHS.

The presence of a VHS has been confirmed in the cuprate $\mathrm{Bi}-2212$ [2], [3]. This is a two-layer cuprate, in the sense that the unit cell comprises two $\mathrm{CuO}_{2}$ layers weakly coupled together across the separating $\mathrm{Ca}$ layer. The coupling results in a splitting of the VHS into a bonding band VHS and an antibonding band VHS. With increasing hole doping it is the antibonding VHS which the Fermi level first crosses. Originally, the primary aim of this project was to acquire susceptibility measurements through the VHS for a single 
crystal of Bi-2212 and to verify reproducibility in the location of the VHS in the dc magnetic susceptibility, as a function of doping, using a polycrystal. There was also a need for a more credible concentration of data points actually on the VHS because of its very narrow range of visibility.

Control of doping levels is critically important to acquire the magnetic data in the limited region where the VHS is visible. Motivation originated in the work done by Dr James Storey for his $\mathrm{PhD}$ thesis and our consequent experimental work done to assess the validity of resultant theoretical predictions. Behaviour of both the calculated thermopower and dc magnetic susceptibility data had shown excellent agreement with the empirical results as holedoping levels, or critical temperature, of lead-doped Bi-2212 passed through the location of the antibonding VHS of this bilayer HTS. However, the expected rise and fall of the magnitude of the dc susceptibility passing through the location of the VHS was nearly overpowered by a dominant, monotonic rise in the susceptibility magnitude that accompanies increasing oxygen doping of the material. The presence of the VHS was only visible close to its peak over a limited range of doping, over such a restricted range that it has been completely unnoticeable in doping progressions taken in previous experimental work eg [4].

In that first experimental study of the VHS in the magnetic susceptibility, there had also appeared an unexpected peak in the magnitude at a doping state close to the location of the VHS on the still further over-doped side. All efforts to find an experimental cause of the phenomenon failed. It remained unexplained, and was believed possibly not an experimental artefact but rather, a 
characteristic arising from some feature in the sample being measured. Contamination was not found, but even if it had been due to an unrecognised contaminant, the addition would have to be paramagnetic or ferromagnetic, in which case the susceptibility curve should not have fitted as it did into the sequence of shape and peak progression with doping seen in the sample's paramagnetic data curves. Data for these curves extends from $100 \mathrm{~K}$ to $350 \mathrm{~K}$. The VHS peak was found at a $\mathrm{T}_{\mathrm{c}}$ of just above $61 \mathrm{~K}$, and the unexplained anomalous peak appeared at a $T_{c}$ a little less than $59 \mathrm{~K}$.

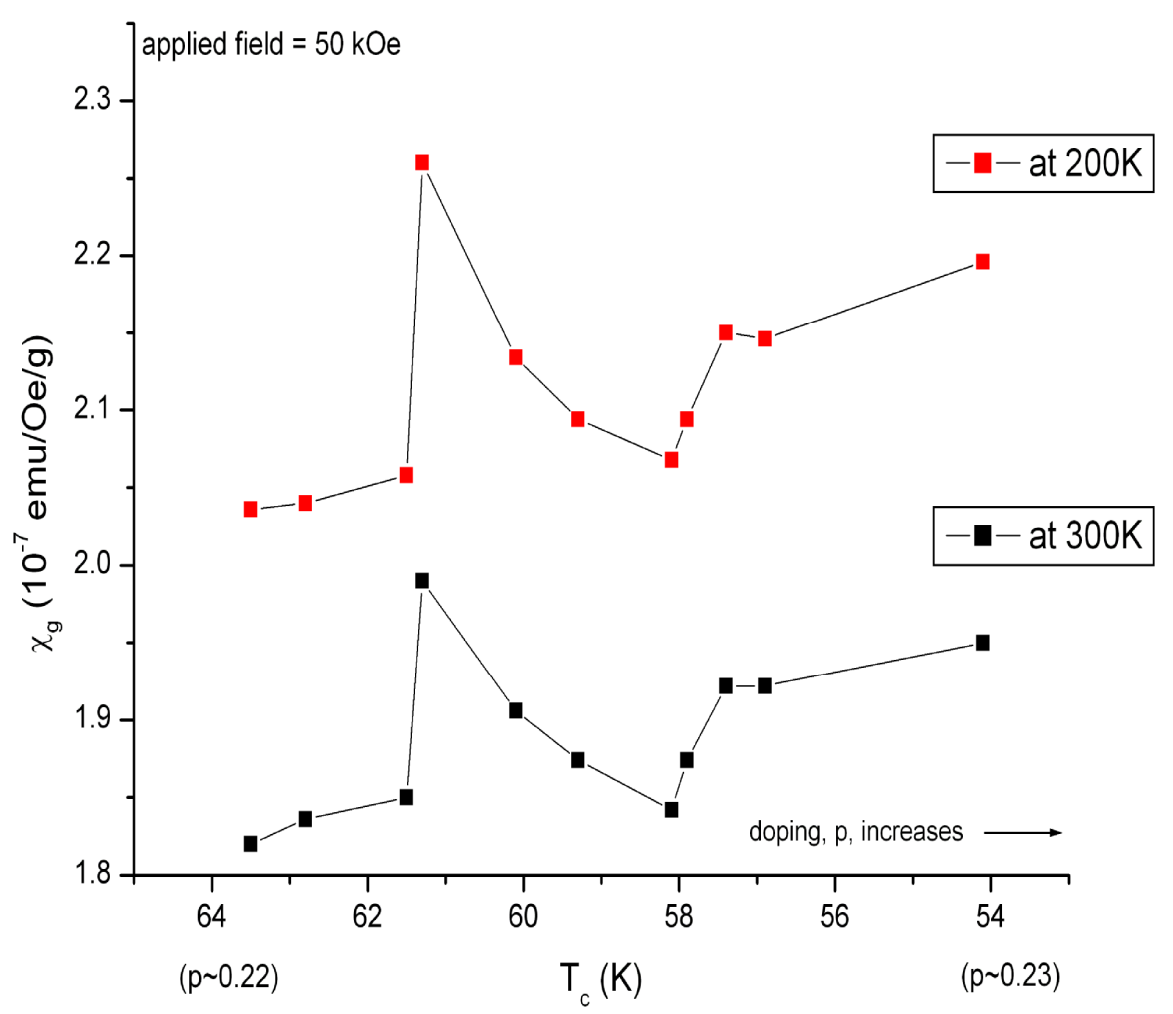

Figure 1. Excerpt from the preliminary view of the VHS in lead-doped Bi-2212. The VHS is clear, but not many data points are actually in the narrow range of $60-62 \mathrm{~K}$ where it is visible. 


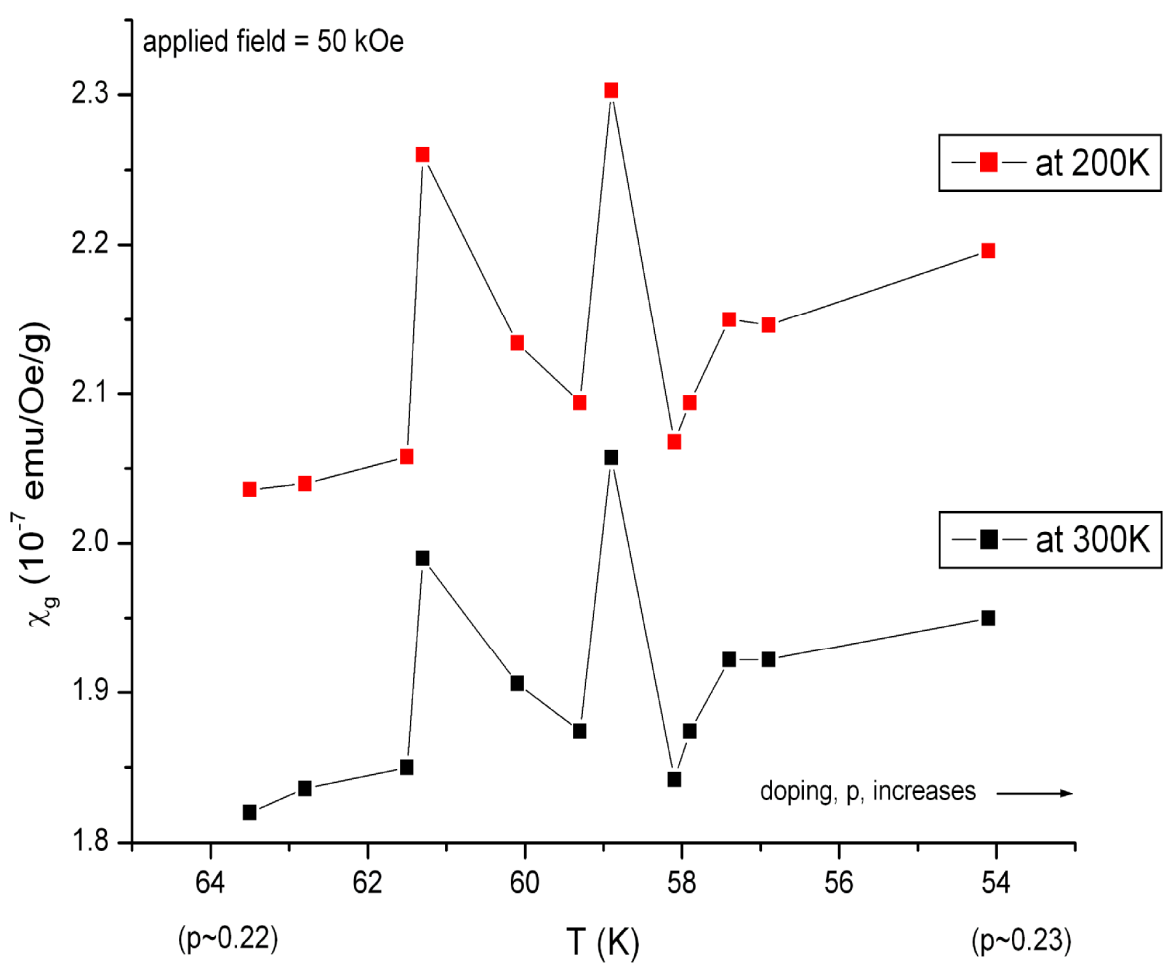

Figure 2. The same view of the VHS with the 'anomaly' included

It was a further hope of this project that some light could be shed on this phantom and verify the presence or absence of this jump in magnitude. It occurred at a doping state beyond the location of the VHS in the region where the sample is more metallic still. Neither the sudden jump in the susceptibility at $\mathrm{T}_{\mathrm{c}} \approx 59 \mathrm{~K}$ nor the background monotonic increase with increasing doping has been predicted.

With increasing experience of empirical observation of the VHS, motivation mounted to strip the cuprates right back to the physics behind fundamental electronics as a foundation for building an understanding of what is happening in the HTS materials. 
In the quest to discern the origin of the charge carrier pairing of superconductivity and ultimately design HTS, understanding of the phase diagram is important. After many years of dedication to the underdoped region of the phase diagram of the cuprates, interest is now being turned to the extreme overdoped regime. It is the goal of this work to identify characteristics of the overdoped regime important to consider when embarking on the study of this region of the phase diagram and to offer an initial look at the cuprates from the perspective of the VHS. 


\section{Introduction}

High Temperature Superconductors have now been studied for many years from the vantage point of generous resources of time, education, experience and funding. They have inspired not only specialist scientists but the general public too. Yet, the excitement and flurry of activity has still not resulted in a fundamental understanding of the nature of how this electrical superconductivity works.

Cuprates still have the highest $T_{c} s$. As more and more substances, both elements and compounds, are found to be superconducting given the right conditions, the view has been raised [5] of the possibility that most 'things' might superconduct if we only knew the condition for that matter to become superconducting. Instead of thinking we have reached the limits of $T_{c}$, there are people beginning to think higher temperature superconductivity is there for the taking - again, if only we knew what to do [6].

Looking back on the 23 years since the discovery of HTS it does seem that the lack of progress is not for want of acquisition of data, or the intelligence, training and experience to interpret it. In spite of this there remains no (general) agreement on the fundamentals of understanding the phenomenon of HTS. Certainly, no one has demonstrated an understanding that stands up to the test of designing a superconductor to predetermined requirements. However, the HTS community has reached agreement on some matters: Firstly, all are agreed that the superconductors we consider operational at 'high temperatures' 
are layered materials. Secondly, that the materials of HTS are highly correlated systems with electrons of a tightly-bound nature. Thirdly, that they are d-wave superconductors with nodes at the zone diagonal and antinodes at the edge of the Brillouin zone. Fourthly, there is wide agreement that there is scant agreement (even if there is little consensus on the level of disagreement). Such agreed facts can be used as clues in the continued superconductivity quest.

To start with the fourth point; it certainly can seem significant that years of effort and resources have not resulted in a deeper understanding. In the most simple form, two main types of conclusions result: One: The physics involved is very strange, of a kind previously unimagined, or Two: We need a new look on familiar physics.

Within the layered structure of the HTS compounds it is believed that one layer acts as a charge reservoir and the $\mathrm{CuO}_{2}$ cuprate layer provides a superconducting plane. This interaction is in itself a good reason to consider a macroscopic view as a starting point.

Yet a belief that it is the copper oxide layers that hold both the key to the understanding and the origin of the superconductivity has resulted in exclusive attention being paid to this layer. Tempting though it may be to seek to discover an exotic new physics, this research trend may have the effect of contributing to the reasons progress is not forthcoming. By narrowing the focus too early it could be all too easy to miss out in a situation where multiple contributions combine to form a unique convergence of known effects. 
For many years, the familiar Fermi "nearly-free" electron theory has served so well for so many circumstances, as noted by Manyala et al. [7]. It is comparatively recently we are faced with important materials where electrons are highly localised, or strongly correlated.

The matter of its occurring in a highly correlated system brings its own difficulties in pinpointing effects important to the superconductivity. A phenomenon that does not have direct control of formation of electron pairs may well have significant influence over another phenomenon that does mediate pairing and may therefore vary in sympathy with $T_{c}$ while not itself being a causal agent. This is all the more reason to take an integrated approach to the cuprates rather than concentrate on specific interactions within a specific plane.

In addition, theory on the Mott parent compound, that appears a fundamental characteristic of the HTS, is not well progressed. Hubbard did a fine job to kick start theoretical understanding of Mott insulators, which, though predicted to be conductors by the conventional band theory for free electrons, are insulators due to the doubling of the unit cell arising from antiferromagnetic correlation [8], [9]. However, he achieved this by assuming great simplifications for the correlated nature of the tightly-bound state. This was definitely an excellent idea, the issue being that even with great simplification the equations remain troublesome to solve analytically and difficult to interpret physically. 
Magnetism, for all its mystery and relation to correlated spins at the microscopic level, is frequently viewed as a macroscopic phenomenon. Many spin arrangements require long-range order. Along with the matter of the HTS being layered materials, magnetism as a macroscopic characteristic increases the need for a holistic approach. It is not necessarily that concentration on a single layer can take into account why the whole provides such a good superconductor when the parts do not. While the traditional view of the cuprates is to focus on the $2 \mathrm{D}$ electron dynamics of individual $\mathrm{CuO}_{2}$ layers, it is essential to take the macroscopic view of a nominally 3D system comprising quasi-2D layers coupled together by Josephson interlayer coupling. This coupling is weak at low doping but essential for superconductivity on the one hand, while at higher doping when the coupling becomes strong (see e.g. [10], [11]), superconductivity is destroyed again. More importantly, as argued by Ashcroft [12], the $\mathrm{CuO}_{2}$ layer, being charged, is not a thermodynamic entity. One must incorporate charge-compensating layers to achieve a true thermodynamic system. This insists that bosonic states in the superconducting intermediate layers must be coupled to the electronic system and the bulk cuprate is essential to its fundamental physics, including superconductivity. This is a rigorous, but overlooked, conclusion.

The layered cuprates provide a rich variety of physics. At the time of their discovery, as well as the unprecedented high temperatures of the superconductivity, the materials displayed the still novel and not well understood characteristics of antiferromagnetic Mott insulators of tightly-bound electrons, and electron dynamics of a 2D nature. The coexistence of such phenomena has made the task of distinguishing those characteristics that cause 
superconductivity from those that coincide with superconductivity a difficult one for scientists. The next chapter gives a brief overview of the cuprates using Bi-2212 as an example. Then chapter 4 reviews fundamental physics that should remain relevant to understanding superconductivity within the framework of the new behaviours. Discussion of the significance of the presence of a $2 \mathrm{D}$ VHS is included. In chapter 5 , the BSCCO samples used in the experimental work are introduced. Attention is paid to the copper oxide layer and detailed description is provided of the strength and variability of the magnetic properties of oxygen, the dopant used to change the charge carrier concentration to show the presence of the VHS. Chapter 6 presents experimental results of the magnetic response of the BSCCO samples, along with magnetic data from other sources, to clarify the presence of the VHS in the cuprates and possible consequences of the use of oxygen as a dopant. Other people's research forms the basis of chapter 7 . Empirical results are compiled that show a link between resistivity and superconductivity. Information from high pressure studies of geophysics demonstrates there does exist an equivalence between doping and pressure. Deliberately, a broad view is taken.

Chapter 8 discusses the VHS in the cuprates, the metal-insulator transition (MIT), and the physical requirements for the formation of superconductivity. Note is made of the further attributes of the cuprates about which little is understood. Finally, chapter 9 summarises what can we conclude from it all. 


\section{HTS and the Cuprates}

\subsection{The HTS past ...}

Until its discovery in ceramic oxides, announced in 1986 by Bednorz and Müller, superconductivity had only been seen in metals and metal alloys at the chilly temperatures of less than 23K. Broadly speaking, HTS refers to superconductivity at temperatures greater than $30 \mathrm{~K}$. The prospect of new materials reanimated interest in superconductivity and after the discovery of Bednorz and Müller it took very little time for $T_{c}$ to be lifted to higher than the temperature of liquid nitrogen by the discovery of new but related cuprate materials. However, such superconductivity has only been found in layered ceramics with the vast majority being cuprates, and recently found in the iron arsenides, again layered materials. Thus all known high temperature superconductors are layered materials with the superconductivity originating in one of the layers. In the cuprates this layer is the square planar copper oxide one with stoichiometry $\mathrm{CuO}_{2}$. Most cuprates have one or two copper oxide layers per unit cell, but can have three or more.

It was confirmed early on [13] that the electrical conductivity of the cuprates is from movement of electrons with the superfluid state being caused, as in the conventional superconductors, by the uniting of the charge into bosonic Cooper pairs. However, the cause of the pairing in the HTS is still not known. 
The parent compound of the cuprates has long been known to be a Mott insulator e.g. [14], a state found in the transition metal oxides. Mott insulators were expected to be metallic, especially at low temperatures, according to the familiar band theory for free electrons, but actually are not because of their antiferromagnetic ordering which puts two charge carriers in the first Brillouin zone, instead of one. The parent Mott insulator is doped to reach the superconducting phase and with still further doping leaves the superconducting phase behind. There exist both hole-doped and electron-doped cuprates. The hole-doped cuprates have the highest $T_{c} S$ and are the most studied.

\subsection{An example of a HTS - the cuprate Bi-2212}

For this project the focus is on the cuprate $\mathrm{Bi}_{2} \mathrm{Sr}_{2} \mathrm{CaCu}_{2} \mathrm{O}_{8+\delta}$, abbreviated as $\mathrm{Bi}-2212$. Its structure is shown in Figure 3 . The bismuth strontium calcium copper oxide ceramics (often referred to as BSCCO) can, like most cuprates, be doped through a wide range of critical temperatures, equivalent to shifting the Fermi level, or chemical potential*, through a range of energies. The basic general formula of $\mathrm{BSCCO}$ is given by $\mathrm{Bi}_{2} \mathrm{Sr}_{2} \mathrm{Ca}_{n}$ ${ }_{1} \mathrm{Cu}_{n} \mathrm{O}_{2 n+4+\delta}$. $\mathrm{BSCCO}$ is hole doped. Increase in the subscript $\delta$ describes the increase in doping as the oxygen content changes.

\footnotetext{
* Strictly speaking it is the chemical potential. In physics, particularly semiconductor physics, many works use 'Fermi energy' to refer to the 'chemical potential' at finite temperature as well as at $0 \mathrm{~K}$. In common with this convention we use in this work the term 'Fermi energy' for finite temperature chemical potential.
} 
A
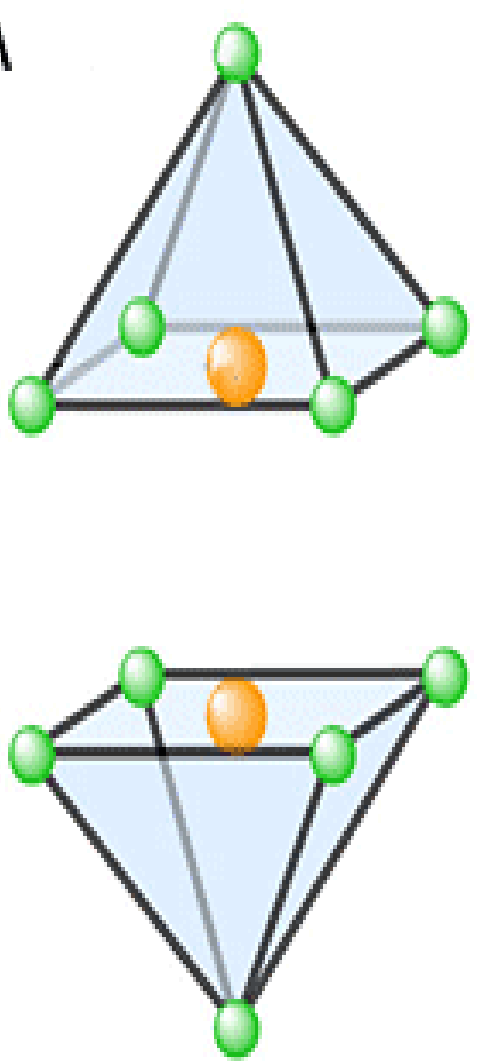

B

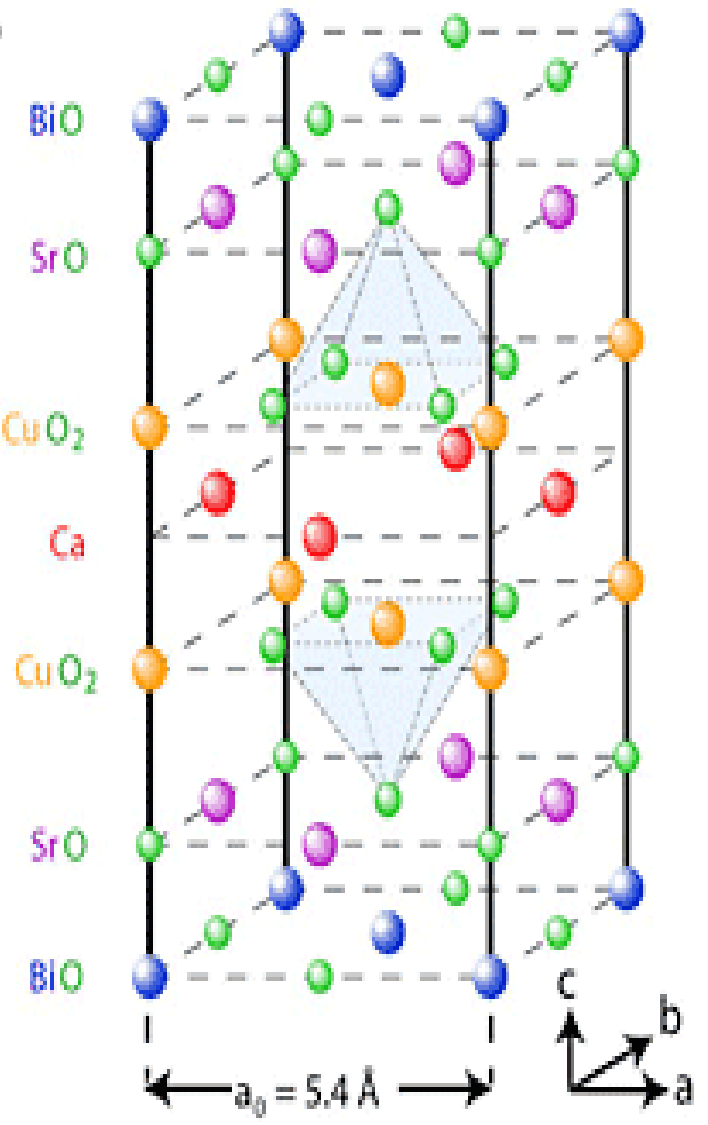

Figure 3. A. The perovskite tetrahedra. B. The unit cell of Bi-2212. . Reproduced with permission from [15]. Copyright 2008 National Academy of Sciences, U.S.A. 
The superconducting cuprates have a generic phase diagram as displayed in Figure 4, that well describes all members in the series $\mathrm{Bi}_{2} \mathrm{Sr}_{2} \mathrm{Ca}_{n-1} \mathrm{Cu}_{n} \mathrm{O}_{2 n+4+\delta}$ :

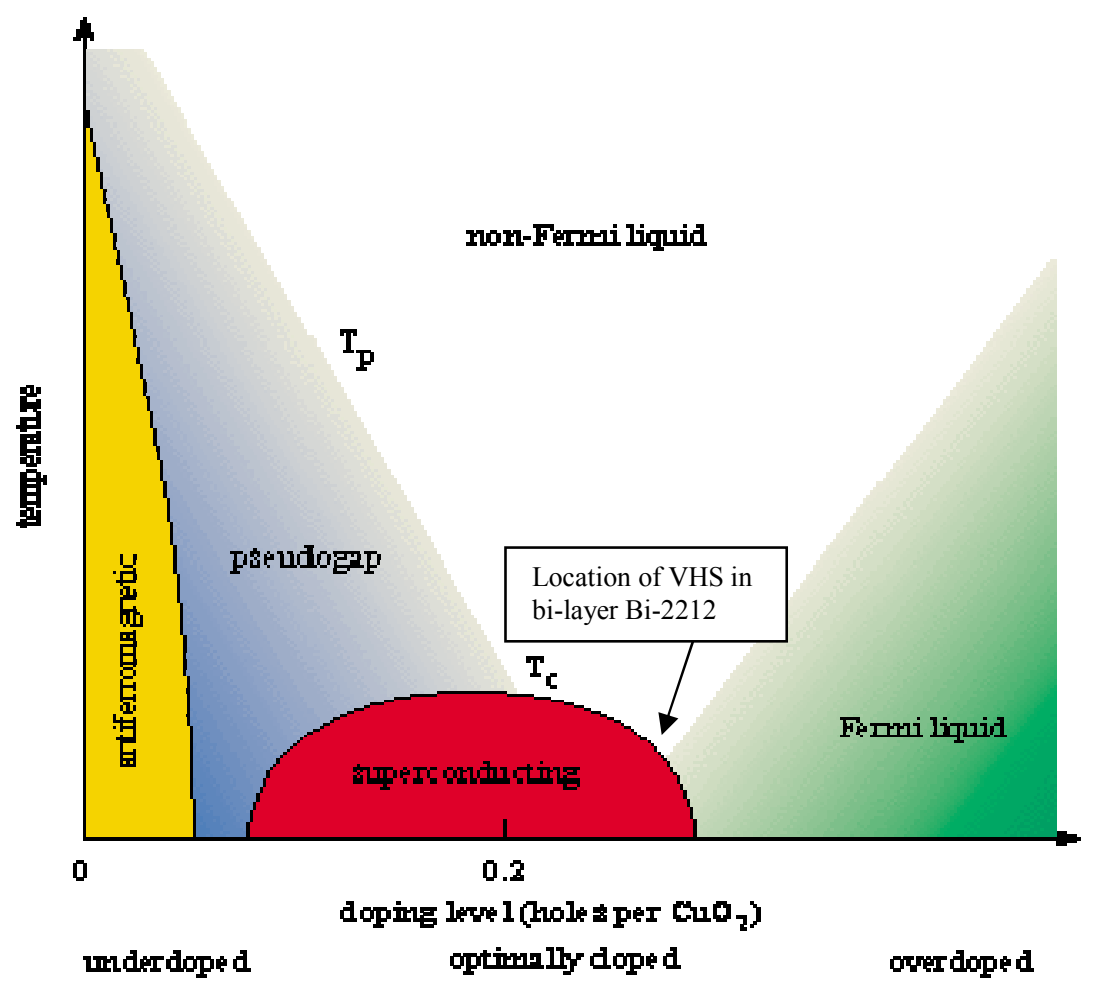

Figure 4. Generic phase diagram for hole-doped cuprates. Phase diagram reproduced from [16], copyright 2000 of IOP. VHS label added. The doping level of the antibonding VHS of the bilayer cuprate $\mathrm{Bi}-2212$ is indicated by the arrow. The phase diagram for the electron-doped cuprates is in essence the same reversed left to right, but with the regions in differing proportions.

The phase diagram is traditionally presented temperature $(T)$ versus doping $(p)$ to show the progression from antiferromagnetic insulator to metallic Fermi liquid. That is, doping marks a development from high resistivity to low resistivity. Leaving aside the superconducting dome for the moment, the phase diagram could be presented T versus resistivity $(\rho)$ as the material is doped from the insulating through to the metallic regimes. Further, in the 
overdoped superconducting region it could be represented as $T$ versus $T_{c}$, where decreasing $T_{c}$ corresponds to increasing doping. We do this from time to time.

In the past, the first metals and various alloys found to comprise the type I and II superconductors have metallic conduction in the normal state. The BCS theory of superconductivity assumes that the normal state above $T_{c}$ be metallic, i.e. that the electron states are extended plane wave propagating states. In this regard the cuprates are extremely interesting in that in the underdoped region the ground state of the normal state appears to be insulating.

\section{$3.3 \ldots$ And the HTS future}

At some stage in the future the issues will hopefully be resolved and we will have a fundamental theory and understanding of the HTS. T.M. Rice commented about the nature of such an achievement:

"Lastly, we can ask what the final theory should predict. First, it should describe the full complex phase diagram. Second, it should reveal the special conditions in the cuprates that lead to this very special behaviour. From this should follow some suggestions for other materials that would show similar behaviour. While it may not be possible to predict $T_{c}$ accurately - because, for instance, of a lack of precise input parameters - the final theory should give the correct order of magnitude and explain the trends that are observed in the cuprates. These trends include the increase in $\mathrm{T}_{\mathrm{c}}$ as we move from single-layer cuprates to those containing two and three copper-oxide layers. These challenges are likely to keep theorists busy for years to come, but hopefully not another decade and a half." - Rice [17].

This was 9 years ago. So far we have not attained this goal. 


\section{Physical Background}

\subsection{Electron pairing.}

Electron pairing was first noticed in the conventional superconductors. As a consequence of the need for an explanation of the observed phenomenon Leon Cooper [18] formulated a mathematical description of a situation where it would in fact be energetically favourable for two single electrons to unite and function as a single unit with constant total momentum and spin. Such a two-electron combination is called a "Cooper pair". In essence, what he found was a phonon-mediated pairing where the pair maintained the same total momentum for the duration of their partnership. Superconductivity in conventional systems arises from this pairing of electrons with opposite spin $(\uparrow \downarrow)$ and opposite momenta (+k, $-\underline{\mathbf{k}}$ ). With total spin of 0 (singlet pairing) the composite electron pair is effectively a boson, so many pairs may occupy the same quantum state. The binding energy between such a pair is greatest when the total momentum is zero, but very quickly drops to zero for a finite total.

In practice the energy and momentum states of electrons are quantised. This restricts the possible sum of momenta of paired electrons. States able to participate are further restricted by the orbitals actually present in materials under consideration, which impose symmetry constraints. Bardeen, an electronics expert, Cooper and Schreiffer, a PhD candidate, combined all these ideas to develop the so-called BCS theory [19], and it served well to explain the 45 -year-old puzzle of superconductivity. It was found 
that the only possible total momentum for an electron pair in the first generation superconductors is zero. There remains the possibility of other total momenta, other total spin and other mechanisms of pairing.

Nearly 100 years after the discovery of the first superconductors in 1911 researchers are still trying to find the pairing mechanism for the high temperature cuprates, and other newly-arrived layered superconducting compounds. That the superconductivity in the HTS compounds is also due to pairs of charge was established quite early, as per various refs in [13]. This is one of the fundamentals that unite all known types of superconducting materials. The pairs are bosons and able to move through a material without resistance because of the opening of an energy gap between the ground state and the first excited state. There are no states available to scatter into and therefore no resistance.

Recently, evidence of electron pairing was found in the insulating state. Amorphous bismuth film patterned with holes can be tuned between insulating and superconducting by changing the film thickness. Stewart et. al. [20] found the insulating state demonstrated, " activated resistances and magnetoresistance oscillations dictated by the superconducting flux quantum $\mathrm{h} / 2 \mathrm{e}$." Charge pairing and electrical conductivity may therefore be distinct characteristics.

\subsection{Bosons}

Bosons are distinguished by their ability to crowd into the same quantum state. This is the crux of superconductivity and superfluidity. However, that bosons can occupy the same quantum 
state does not mean they will be doing so in a given situation. Also, bosons of paired electrons are composed of fermions, particles that must never occupy the same quantum state. Fermions must distribute over the states available in such a way that two at most, with opposite spin, occupy the same energy and momentum state. The momentum total of any two fermions thus has a dependence on the availability of the states within a material.

\subsection{Density of States}

In a material with 2D characteristics, such as the cuprates, the DOS is the number of states able to be occupied per unit of kspace area per unit energy. The symmetry of the underlying square lattice modifies the nearly-free electron parabolic dispersion, to be very shallow at the Brillouin zone boundary and very deep at the zone diagonal, as shown in Figure 5. With doping, the Fermi energy falls until there is a cross-over from holelike to electron-like conductivity. This change in majority charge carrier is accompanied by a peak in the DOS associated with the "saddle-point" at the zone boundary.

For $\mathrm{Bi}-2212$, angle resolved photoemission spectroscopy (ARPES) was used to measure the dispersion shown in Figure 5. ARPES is based on the photoelectric effect and is particularly suitable for 2D momentum maps. The incoming photon has negligible momentum, and the momentum of the electron parallel to the surface is unchanged, so the energy characteristic of the ejected electron [21] may be easily determined. 
It is easier to see the peak in the DOS (where the dispersion has a saddle point) in 1D, and this is shown in Figure 6, but the principle is the same for the 2D DOS. The dispersion of Figure 5 was the one used to calculate the DOS that occurs in the theoretical predictions for the behaviour of the magnetic susceptibility through the location of the VHS. The bonding VHS has a dispersion of similar form at lower energies but only one cone is shown in the illustration.

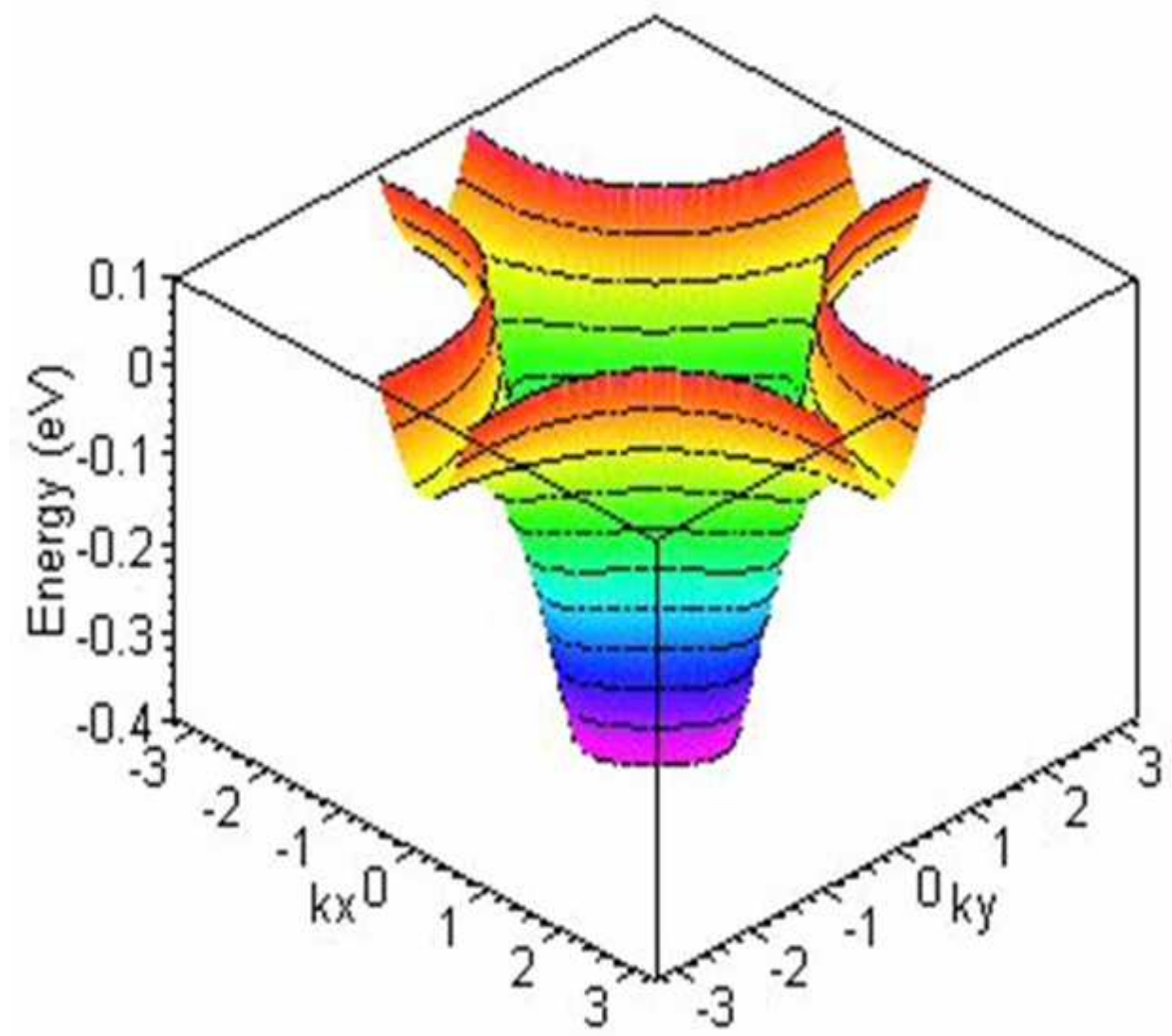

Figure 5. The $\mathrm{Bi}-2212$ dispersion according to a tight-binding fit of ARPES data [22] from $\mathrm{Bi}_{2} \mathrm{Sr}_{2} \mathrm{CaCu}_{2} \mathrm{O}_{8}$. The site of the $\mathrm{VHS}$ can be seen at the edges of the zone where the derivative is zero in both the $\mathrm{k}_{\mathrm{x}}$ and $\mathrm{k}_{\mathrm{y}}$ directions. Presentation of dispersion is reproduced with permission of Dr. James Storey, Copyright 2008 [23]. 


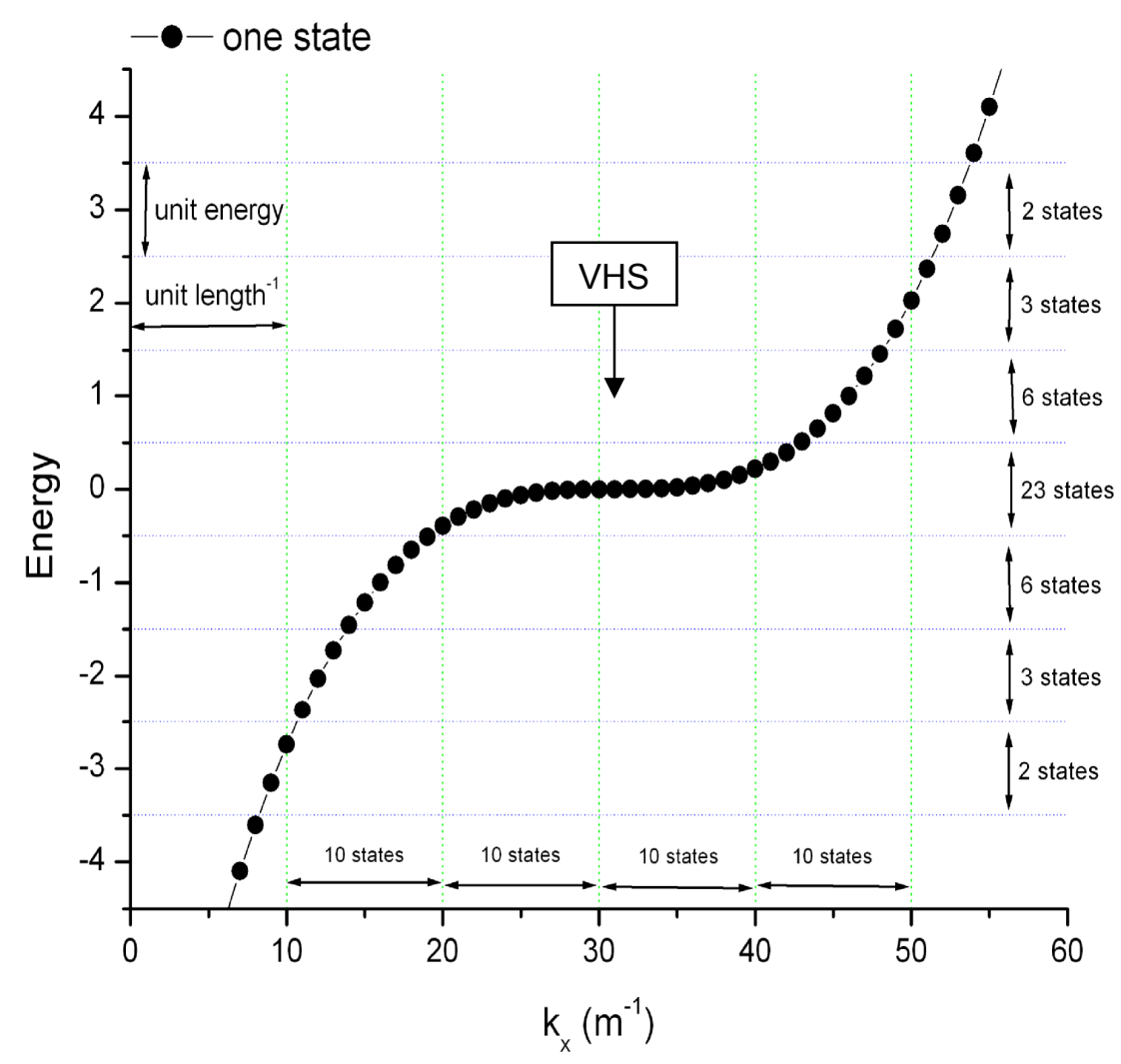

Figure 6. Density of states depicted in 1D. The states bunch up over the flattened region around a VHS causing a peak in the DOS. We can obtain the same dispersion curve by doing a cut in Figure 5 from $(0,0)$ to $(\pi, 0)$, then to $(\pi, \pi)$.

Theoretical predictions for the effect of the DOS on the susceptibility can be made assuming a Fermi liquid of weakly interacting holes or electrons with spin susceptibility, $\chi$, given by:

$$
\chi=2 \mu_{B}{ }^{2} \int \frac{\partial f(\varepsilon)}{\partial \varepsilon} N(\varepsilon) d \varepsilon
$$

where $f(\varepsilon)$ is the Fermi function, $N(\varepsilon)$ is the density of states DOS, $\varepsilon$ normal-state energy and $\mu_{\mathrm{B}}$ the Bohr magneton. 
The general theoretical background of the equation is described generally in Ashcroft and Mermin [24] and applied to the specific situation of the Bi-2212 by Storey et al. [25]

The DOS figures prominently in the expression and a peak in the DOS is traced out in the magnitude of (dc) magnetic susceptibility data. The theory was successfully used [25] to identify the location of the antibonding VHS in the Bi-2212. We found it at the same location (within experimental uncertainty) in (Bi,Pb)-2212 samples. Figures 7 and 8 , in the next section on the VHS, display calculated theoretical predictions for the magnetic susceptibility.

\subsection{Van Hove Singularity}

Generalising as much as possible, Van Hove showed that geometry is important in effects on the DOS [1]. A saddle shape in the dispersion of energy (frequencies) leads to a singularity in the DOS of a crystal with an effective 2D geometry. A 2D VHS occurs in the middle of a band. The singularity that occurs there as the result of such a VHS thus has a strong effect on the electrons (the fermions that make up the Cooper-pair bosons), and therefore also affects conductivity - super or not.

A change of majority charge carrier is an important physical consequence of crossing the 2D VHS where there is a change from hole-like to electron-like conductivity, or vice versa. This occurs because the Fermi surface changes from one which is centred on the Brillouin zone boundary to one which is centred on the zone centre. Thus e.g. in a magnetic field the electron orbits around the Fermi surface are of opposite sign either side of the 
VHS reflecting the hole-like or electron-like character of the carriers. Until recently our most familiar electronic systems were three dimensional. Now, low-dimensional systems are becoming important. All known high- $\mathrm{T}_{\mathrm{c}}$ systems are quasi $2 \mathrm{D}$, with only weak coupling normal to the $\mathrm{CuO}_{2}$ planes.

Markiewicz [26] does a highly creditable job of summarising knowledge of the 2D VHS characteristics while maintaining a distinction between the phenomenon and the material in which it appears. To his own statement, "The VHS can explain a number of normal-state anomalies in the cuprates, including the linear-in-T and $\omega$ electron-electron scattering rate and a pronounced crossover in properties from the underdoped to the overdoped regime", he answers:

"Many researchers have noted that, whereas the superconductivity of the cuprates is relatively conventional, except for the high Tc values and the (probably) d-wave symmetry of the gap, the normal state properties are highly anomalous, and a key to understanding the cuprates is to understand these anomalous normal state properties. In this respect, the fact that the normal state anomalies follow rather naturally from the proximity of the VHS is very significant."

In their work using ARPES to map the Fermi surface of $\mathrm{Bi}_{2}\left(\mathrm{Sr}_{0.97} \mathrm{Pr}_{0.03}\right)_{2} \mathrm{CuO}_{6+\delta} \mathrm{King}$ et al. [27] find an extended saddle point at or near the Fermi level and note; "This agrees with earlier photoemission data from the cuprates which reveals a striking correspondence between the position of the Fermi level relative to the saddle-point singularity and their normal state properties; however, it does not support the existence of a simple link to a high $\mathrm{T}_{\mathrm{c} .}$ " 
This latter point could be traversed in more detail (they are referring to lack of success in linking the magnitude of $T_{c}$ to the relationship between the Fermi level and the VHS), but the main issue is, the cuprates are materials of interconnected (2D) layers, therefore they have a 2D VHS. Since it is there - and since it is of a 2D nature - the VHS will naturally have considerable impact on the electronic properties of the system. That the system is also capable of superconductivity may be for no other reason than different phenomena can occur in the same material. There seems a paucity of evaluation of the cuprates from the consideration they would have a VHS anyway. That a material with a 2D VHS display (normal state) properties that result from a 2D VHS should not come as a surprise!

We should also like to point out it follows from the divergence in the DOS that the ground state at a 2D VHS has an unstable Fermi surface [26]. The transition between hole-like and electron-like conduction has a point where it is neither one nor the other. It could be described as a point of "reconstruction" of the Fermi surface. A phase transition could ensue, or abrupt changes in thermodynamic state. There could be a structural rearrangement that splits the VHS and therefore lowers the electronic free energy.

Materials with one $\mathrm{CuO}_{2}$ layer per unit cell have one VHS. As noted, cuprates such as $\mathrm{Bi}-2212$, with two $\mathrm{CuO}_{2}$ layers in the unit cell, have two VHSs. When there are two layers, bilayer splitting divides the VHS into two parts: the bonding VHS, and the antibonding VHS. It is the antibonding VHS that lies in the range of easily accessible doping in the Bi-2212. 
As the doping causes the Fermi level of the BSCCO to pass through the location (of the energy) of the VHS, corresponding changes occur in certain properties such as the dc magnetic susceptibility. This is therefore a good characteristic to measure to confirm location of a VHS with respect to $T_{c}$, or doping. Equation (1) was used for calculations, done by Dr James Storey [23], that predict such attributes as location of the VHS and the shape of a typical susceptibility curve of the material. Figures 7 and 8 illustrate the theoretical expectations for the antibonding VHS of Bi-2212 (with no lead).

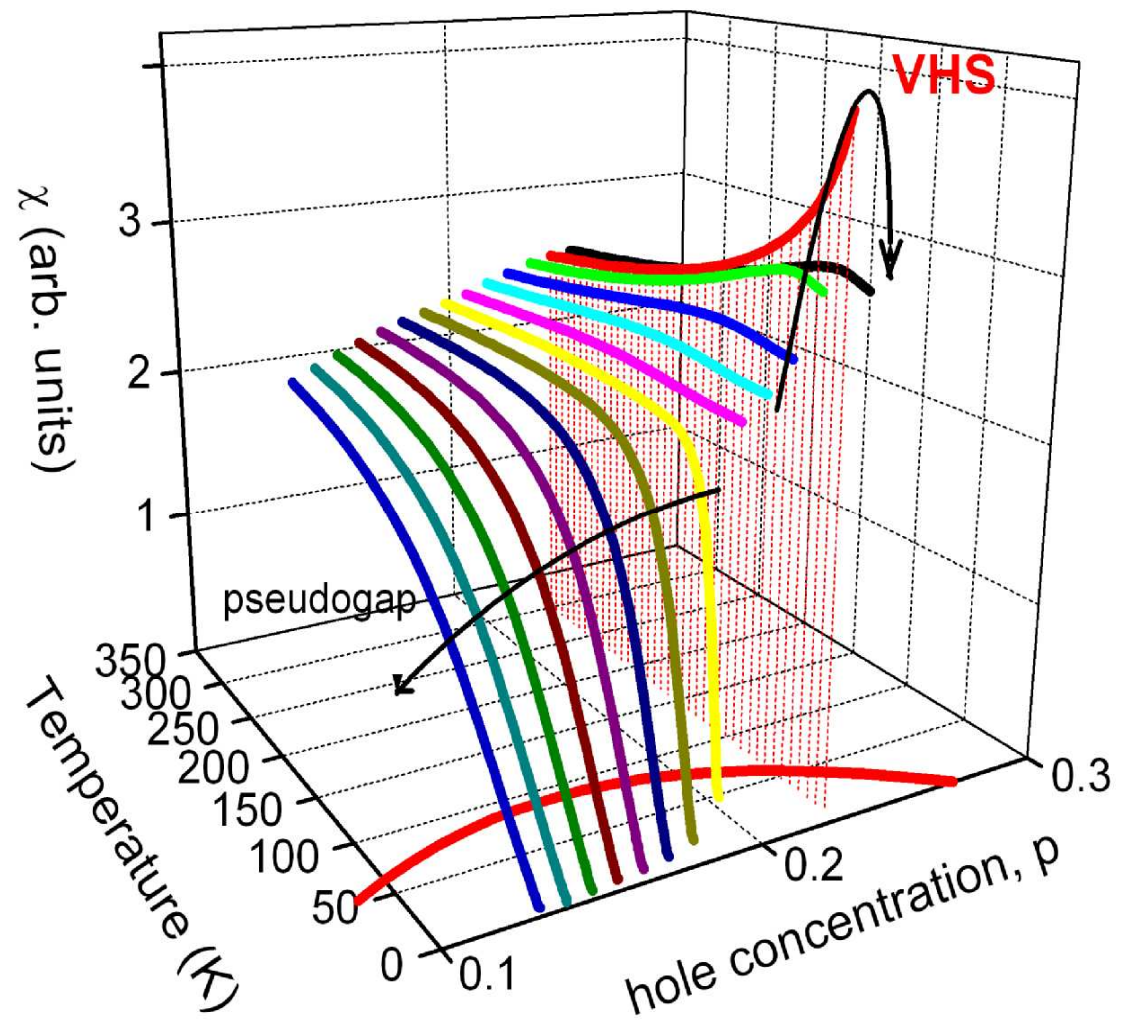

Figure 7. Theoretical prediction showing the effect on the magnetic susceptibility response of $\mathrm{Bi}-2212$ of the VHS found at doping level of around 0.225 holes per copper atom. Graph from [28], adapted with permission of $\mathrm{Dr}$ James Storey. 


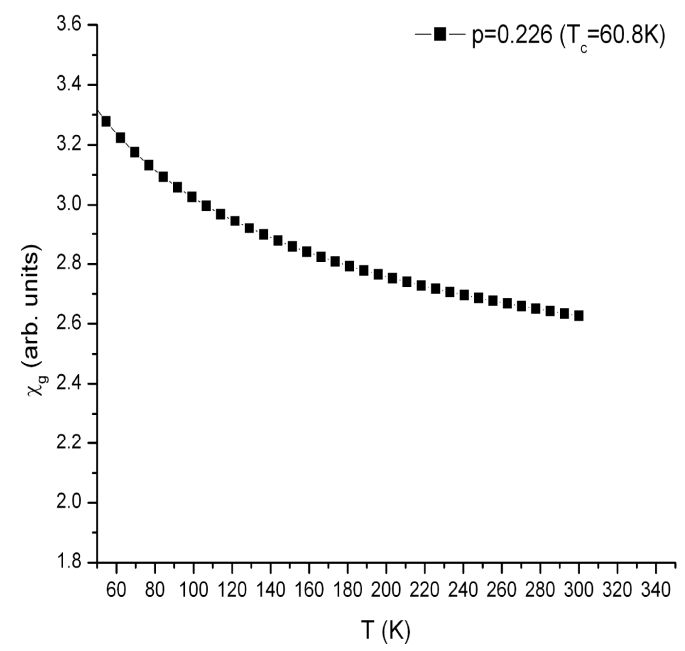

Figure 8. Side view of one of the susceptibility curves: for one particular doping as a function of temperature. With permission [28].

Many attempts have been made to either equate the VHS directly with pairing or to describe its use in enhancing $T_{c}$. e.g. [29], [30].

Other work concludes the VHS to be of little consequence in the formation of pairs e.g. [31], [32].

In BCS theory the relationship between $\mathrm{T}_{\mathrm{C}}$ and the VHS is contained in the expression for nearly-free electrons:

$$
T_{c} \propto \hbar \omega_{B} \exp \left(\frac{-1}{N(\varepsilon) V}\right)
$$

where the DOS has the symbol $N, V$ is the pairing strength and $\omega_{B}$ is the corresponding pairing boson frequency. $T_{c}$ is exponentially dependent on the inverse DOS, which in turn is markedly affected by the presence of a VHS. However, questions remain about the impact of the pairing mechanism, boson cut-off frequency and indeed, whether BCS theory is applicable to the cuprates. 
It appears to the writer much of HTS research has been carried out from the point of view that a high DOS equates to pair formation. So since a (2D) VHS has a phenomenal effect on the DOS, this has naturally helped lead to consideration of a VHS pairing mechanism. The success of the VHS scenario in explaining the unusual normalstate properties of the cuprates has been seen as support for its continuing use in efforts to explain their pairing mechanism. It may well be that a $2 \mathrm{D}$ VHS is an integral part of any system constructed so it can superconduct in the way the cuprates can. However, the VHS can still be incidental to the actual mechanism of pair formation.

\subsection{Conductivity}

At its most fundamental level conductivity arises when charge is able to move within a material.

In metals electrical conductivity is provided by a sea of nearly-free electrons, loosely bound in plane-wave states. An electric current flows when there is a potential difference on either side of the sea.

In p-type semiconductors, electrons densely occupy the states through the valence band. Doping removes electrons from the band, which is equivalent to increasing the number of vacancies, or 'holes'. As the electrons are transported, so are the holes, and the movement of these holes is what is detected as the current. For the holes to maintain coherence as the current flows, the electrons that form them must conduct themselves in an orderly fashion, i.e. they 
must not be heavily scattered - they must have a lifetime which exceeds the timescale of the experimental technique.

As further electrons are removed, more holes appear, and the current, or conductivity, increases. When electrons continue to be removed, or holes doped in, the balance eventually shifts and the holes outnumber the electrons. With the change in ratio the material passes though a crossover between hole-like and electron-like flow.

Hole-doping also disrupts antiferromagnetism as charge, and its spin, is removed. In particular, the spins associated with the holes doped into oxygen orbitals frustrate the antiferromagnetism and the Neél temperature, $T_{N}$, falls rapidly.

As well as doping there is thermal excitation with increasing temperature. The conduction band becomes accessible. Holes can also appear in the valence band due to electrons becoming excited into the conduction band. It is not necessarily a given that the holes from both doping and thermal excitations will result in electron pairing. Thermal excitations are pairbreaking, but doping and thermal activation do both contribute to change to electron-like conduction.

The ultra low resistance of superconductors is a distraction from the nature of "good" conductivity. Deutscher remarked, [33] "It [BCS theory] was even used by some to predict that high temperature superconductivity could not exist. In fact, it is probably a correct prediction that metals cannot be high temperature superconductors." 
As the balance passes through the exactly half-and-half split of hole and electron charge carrier the conductivity is (for the moment) weighted neither towards showing hole-type conduction nor electrontype conduction, and it is here in the 2D materials the physical conditions occur for the singularity in the density of states.

\subsection{Resistivity}

A good insulator is characterised by a large band gap. Generally, insulating materials have a full valence band with two electrons, spin up and spin down, per state. With a smaller bandgap the material may be classified as a semiconductor and is often doped to achieve working levels of conductivity. On continuing doping a semiconductor can become "degenerate" where the conducting behaviour ceases to be insulator-like and takes on metallic characteristics.

As well as providing charge carriers, increasing quantities of the dopant may affect the lattice spacing of the background potential. This can lead to a progressively changing relationship between the periodicity of the ions and the electron wavelength - and accordingly vary the bandgap.

The superconducting $T_{c}=1.2 \mathrm{~K}$ of aluminium can be raised by increasing the resistivity [34]. By oxidising the aluminium to partially form aluminium oxide, the resistivity increases and Dynes and Garno found the superconducting $T_{c}$ to rise to a peak then fall away before collapsing on crossing a metal-insulator transition where the screening length diverged. 
The tightly-bound electrons of a Mott insulator also fill the valence band, but with one electron per state. It is expected to be a metal by the nearly-free electron theory. In fact, the spin-up/spin-down arrangement of the antiferromagnetism leads to the doubling of the unit cell which now has two electrons in the Brillouin Zone, and the material is an insulator. In this case, electrons are prevented from mobility by the strong Coulomb repulsion. The cuprates are doped away from the Mott parent insulator, eventually becoming able to superconduct at low temperatures, then lose superconductivity as Fermi metal-like behaviour sets in.

\subsection{Degenerate Semiconductors (i)}

A semiconductor is degenerate when the concentration of impurity charge carriers becomes large compared to the host atoms. As dopant concentration increases from low to high the added charge carriers move closer together. They lose their isolated status and begin to interact with each other, which causes splitting into an energy band. This dopant impurity band closes in on either the valence or conduction band and the material behaves as a conductor. Hole doping moves the impurity band towards the valence band. When the Fermi level moves within $3 \mathrm{k}_{B} T$ of either the conduction or valence band, Fermi-Dirac statistics, rather than Maxwell-Boltzmann, start to apply. Eventually the bands overlap and the electronic structure is as for a metal. Now, the distinction between semiconductor and metal becomes the number of charge carriers, a more subjective criterion. The metal-like semiconductor still has very much fewer charge carriers than the intrinsic metal. 


\subsection{The Metal-Insulator Transition}

There exist two main characteristics that can identify the presence of a MIT:

1) Traditionally the gradient of the resistivity as a function of temperature is the standard used to distinguish the insulatorlike side (negative slope) from the metallic side (positive slope) of the MIT.

2) And in Mott materials, a microscopic inspection shows shorter bond lengths are metallic and longer bond lengths are insulator-like, with a critical length marking the MIT.

The MIT in the cuprates is of interest from two points of view: the vertical temperature-dependent Mott MIT and the horizontal dopingdependent MIT as the material is doped hole-by-hole from excellent insulator through to metallic. The vertical Mott MIT arises from electron-electron interaction and physically occurs at a critical bond length [35]. Its existence is not dimension-dependent. e.g. [36]. For a given substance the Mott MIT is a function of temperature. However, doping can change bond length as well as the number of charge carriers, and also alter the antiferromagnetism. Thus, doping can alter the temperature at which the Mott MIT occurs.

Generally, the appearance of a transition from insulator to (metallike) conductor had been observed in systems where the ratio of the energy of the electron-electron interaction to the Fermi energy is high [37] (the effective mass is high), true for both p-type and n-type 
materials, such as $\mathrm{p}-\mathrm{GaAs} / \mathrm{AlGaAs}$ and $\mathrm{n}$-AlAs heterostructures. Observation of the GaAs-AlGaAs hole gas [38] found a minimum conductance, $\sigma_{\min }=2 \mathrm{e}^{2} / \mathrm{h}$, distinguishing clear insulating and conducting phases. This is the Mott minimum metallic conductivity. The strongly insulating regime fitted resistivity of $\rho(T)=\rho_{0}\left(T_{0} / T\right)^{1 / 2}$ with $\rho_{0}=\mathrm{h} / 2 \mathrm{e}^{2}$.

A MIT can be observed in systems as simple as a conductorinsulator junction such as where a metal, for example aluminium, is in contact with its oxide. In this case the junction(s) can be cooled to also exhibit superconductivity. Or it can occur, as in the cuprates, where the Mott MIT occurs within a single medium without the need to come in contact with another substance, by methods such as doping. Debate continues, however, as to whether HTS are homogeneous. [39], [40]

As a function of temperature the insulating state in a Mott material is commonly the cold state, as in the cuprates, changing to the metallic on heating. It is possible for the reverse to occur. Rubenstein [41] found $\mathrm{V}_{2} \mathrm{O}_{3}$, with a small amount of $\mathrm{Cr}$ dopant, warmed from an antiferromagnetic insulator to a metal, then on further warming changed to a (paramagnetic) insulating state. He found evidence that both MITs are of the Mott type.

The pressure-induced MIT in transition metal compounds is much studied by the earth sciences, where the interest is in the concurrent magnetic transition. 


\subsection{Mott insulators}

Mott insulators were expected to be conductors by the conventional band theory of free electrons. Their electrons strongly feel each other's coulomb repulsion, which has the effect of highly localising them. The antiferromagnetism of the Mott insulator state (of the HTS) comes from the localisation of the electrons in real space. Weak hopping of electrons from site to site insists that the spins are antiferromagnetically ordered so as to accommodate the Pauli exclusion principle. As the electrons cannot occupy the same state, the spin up - spin down arrangement for adjacent sites is needed to maintain distinct sets of quantum numbers between the fermions when an electron hops to its neighbour's site and the two are sharing the same space.

This doubles the size of the unit cell, halving the area of the Brillouin Zone which has two electrons per Zone and thus establishes an insulating ground state.

The resistance of a cold Mott insulator is extremely high. A characteristic of the system is a metal insulator transition where the slope of $R$ vs $T$ crosses over from negative to positive as temperature is increased. The MIT has become of great interest in conventional electronics and the change to a metallic conductivity accompanies the loss of superconductivity in the cuprates as doping is increased e.g. [42]. 
Work has been done that correlates the apical bond length with the ability of the cuprate to superconduct e.g. [15]. It is related to the $T_{c}$ of the material. Bond length is also a variable that controls whether or not a Mott-like material is on the metal or insulator side of the MIT.

\subsection{Magnetism}

Moving charge is the basis of magnetism and of electrical current. Although electrical transport is well understood compared with the mysteries still surrounding magnetism, both require the existence of the electrically charged particles; the lattice ions and the electrons. The spin of an electron is classically analogous to a current loop, with spin 'up' and spin 'down' equivalent to the charge flowing in opposite directions so that the miniature magnet within is pointing either 'up' or 'down'

\subsubsection{Diamagnetism}

Diamagnetism arises from the tendency (Lenz' Law) of the movement of an orbiting charge to react to an applied magnetic field in a way that produces an opposing field. Diamagnetism is the odd man out of the commonly considered magnetic states. The other forms of magnetism stem from the basic tendency of spin to align with an applied magnetic field. Diamagnetism is always present but is a weak response and is normally hidden in the presence of the much stronger alignment of the unpaired spin with the field. It is mostly seen in substances with an even number of electrons where the orbitals are fully occupied (spin up and spin down) and the orbital moments of the electrons sum to zero. However, substances with 
unpaired spins can experience situations that subdue the ability to align with a magnetic field, or be a combination of elements where the unpaired spin is only a minor portion, and the total response measured is diamagnetic. Generally, diamagnetism is viewed as the tendency of a substance to weaken an applied magnetic field, i.e. it has a negative susceptibility.

In superconductivity, the 'perfect' diamagnetism arises from the fieldinduced macroscopically circulating supercurrent, which is strong enough to expel, or oppose, the magnetic field from the interior.

\subsubsection{Paramagnetism}

Paramagnetism, generally, results from random unpaired spins that become aligned in the direction of the applied magnetic field but lose that alignment due to thermal activation when the external field is removed.

\subsubsection{Ferromagnetism}

Ferromagnetism is characterised by unpaired spins that align spontaneously or align in the direction of an applied magnetic field and remain aligned when the external magnetic field is removed. Above the Curie temperature of a material - with enough thermal fluctuation - ferromagnetism becomes paramagnetism.

\subsubsection{Antiferromagnetism}

For the antiferromagnet, the averaged magnetic moment of the bulk substance is close to zero. Close inspection at the microscopic level however, shows the spins are not random at all, but highly ordered with spin up alternating with spin down so that their contributions 
exactly cancel. This is referred to as "staggered magnetisation". Like ferromagnetism, the arrangement is particularly sensitive to increasing temperatures, and when heated above the Néel temperature antiferromagnetic ordering is destroyed by thermal fluctuations and the substance becomes paramagnetic. Antiferromagnetism in crystals can be achieved by two ferromagnetic layers pointing in opposite directions, or by two antiferromagnetic layers which may be aligned so the vertical arrangement is also antiferromagnetic or so the vertical is ferromagnetic walls of alternating directions. Antiferromagnetism is a long range ordering in real space of electron spin.

\subsubsection{Ferrimagnetism}

Ferrimagnetism is characterised by alternating spin up and spin down in the same style as in antiferromagnetism, but where the total magnetic moment of one direction is greater than for the other direction, leaving a residual ferromagnetic response. It is physically seen in materials with ferromagnetic layers where the alignment of neighbouring layers is in opposite directions and of differing magnitude.

A way of looking at a relationship between ferromagnetism, paramagnetism and antiferromagnetism is to express the induced magnetisation as:

$$
M=\frac{C}{T-\theta} H
$$

$M$ is the magnetic moment, $C$ is the Curie coefficient, $H$ is the magnetising field, $\mathrm{T}$ is the temperature in Kelvin. $\theta$ is the Curie temperature. 
With:

$\theta<0$ antiferromagnetism

$\theta=0$ paramagnetism

$\theta>0$ ferromagnetism

Above the Curie point a ferromagnetic material becomes paramagnetic

Above the Néel temperature an antiferromagnetic material is paramagnetic.

Or, on cooling a paramagnetic material can

1) remain paramagnetic,

2) become ferromagnetic,

3) become antiferromagnetic. 


\section{The Bi-2212 material}

\section{1 samples used in this experiment}

Our own previous data for lead-doped Bi-2212 polycrystalline samples clearly showed the presence of the peak of the antibonding VHS in dc magnetic measurements [unpublished data]. The results appeared strong and easily reproducible. Samples for use in this project were obtained from a variety of sources. The main $\mathrm{Bi}_{1.7} \mathrm{~Pb}_{0.3} \mathrm{Sr}_{2} \mathrm{CaCu}_{2} \mathrm{O}_{8+\delta}$ polycrystal was inherited from a previous experiment that resulted from ongoing work. The Bi-2212 single crystal was supplied by C.T. Lin of the Max Planck Institut, Stuttgart on request to have a similar lead content.

We used these samples to search for the antibonding VHS, which arises from the 2D nature of the conducting layer. The separation of the bonding and antibonding VHSs depends on the strength of coupling between the two layers. If these layers are sufficiently separated (or weakly coupled) within a material each VHS occurs at the same energy. However, when the layers become close or strongly coupled, as are the two layers in the Bi-2212 unit cell, there is interference that splits the bands and hence the singularities to accommodate the Pauli principle. The bonding VHS is at the lower energy and the antibonding VHS is located at a higher energy level.

The previous measurements, as well as demonstrating the presence of the VHS peak, also had shown a companion peak in the magnetic susceptibility response of the BSCCO unpredicted and unaccounted 
for by theory. Neither does theory predict a monotonic rise in the dc susceptibility with increasing doping. While the approach to the peak of the bonding VHS would eventually cause the susceptibility to rise again, this increase is not expected to be detectable until around halfway between the singularities. According to calculations using equation (1) we do not expect to see the susceptibility start to rise again until a doping level of around $p=0.26$. Or, we expect to see a monotonic decrease from the VHS until $T_{c}$ gets close to $16.5 \mathrm{~K}$. Only then the magnitude of the susceptibility is expected to start to rise again for the approach to the bonding VHS.

\subsection{The layers}

The precursors that form $\mathrm{Bi}-2212$ could combine as a multi-metal oxide, such as $\mathrm{Bi}_{2} \mathrm{CuO}_{4}$ where it is impossible to traverse from one bismuth atom to another without crossing a copper atom, and vice versa. However, they do not. The metals in the HTS materials are segregated. One of the few areas of widespread consensus about the cuprates is how they are layered materials. When we look at the crystal structure (shown in Figure 3) of the Bi-2212 it consists of the layers

$$
\begin{gathered}
\mathrm{BiO} \\
\mathrm{SrO} \\
\mathrm{CuO}_{2} \\
\mathrm{Ca} \\
\mathrm{CuO}_{2} \\
\mathrm{SrO} \\
\mathrm{BiO} .
\end{gathered}
$$


All being of positive valency, it is hardly surprising the metals do not form chemical bonds with each other, particularly with the relatively low sinter temperatures of the HTS materials and the ready availability of the oxygen. Nevertheless, the result is that even after the boiling off of the carbon-oxide, or nitrogen-oxide gases, from the precursor compounds the metals are left in layers that distinguishes them. Whether the precursor combination is $\mathrm{Bi}_{2} \mathrm{O}_{3}, \mathrm{SrCO}_{3}, \mathrm{CaCO}_{3}$ and $\mathrm{CuO}$; or $\mathrm{Bi}_{2} \mathrm{CuO}_{4}, \mathrm{Sr}_{2} \mathrm{CuO}_{3}$, and $\mathrm{CaCO}_{3}$ or includes nitrates, the metals always form the stable layered structure. In the cuprates the superconductivity occurs along the copper oxide layer.

Doping is usually done by changing the oxygen excess $\delta$ in the structure through different annealing temperatures and oxygen partial pressures. Achieving higher doping levels is aided by substituting $\mathrm{Pb}^{2+}$ on some of the $\mathrm{Bi}^{3+}$ sites. For this reason we use lead doped $\mathrm{Bi}-2212$ to more easily reach the antibonding $\mathrm{VHS}$, the most accessible of the VHS in the Bi-2212. Extra oxygen that is doped into the cuprates goes into the bismuth oxide layers [43] and from this position donates holes into, or accepts electrons from, the copper oxide layers.

Over the years of study of the cuprates interest has been focussed on the $2 \mathrm{D} \mathrm{CuO}_{2}$ layer because of the superconductivity found there. In the parent Mott insulator the copper has a valence of $2^{+}$and as part of the interest in this layer $\mathrm{CuO}$ has been subjected to study. 


\section{$5.3 \mathrm{CuO}_{2}-\mathrm{CuO}$}

The superconductivity of the cuprates is in the $\mathrm{CuO}_{2}$ layers with $\mathrm{Cu}^{2+}$ and because of this $\mathrm{CuO}$ has been of interest. In cupric oxide the onset of antiferromagnetism is at the Néel temperature of $230 \mathrm{~K}$ with an incommensurate structure, without translational symmetry, then changes to commensurate at $213 \mathrm{~K}$ [44], but does not reach its maximum ordering until $140 \mathrm{~K}$, where the magnetic susceptibility drops to its lowest magnitude. Above $140 \mathrm{~K}$ magnetic disorder increases. A schematic diagram of this behaviour in magnetic susceptibility data is shown in Figure 9.

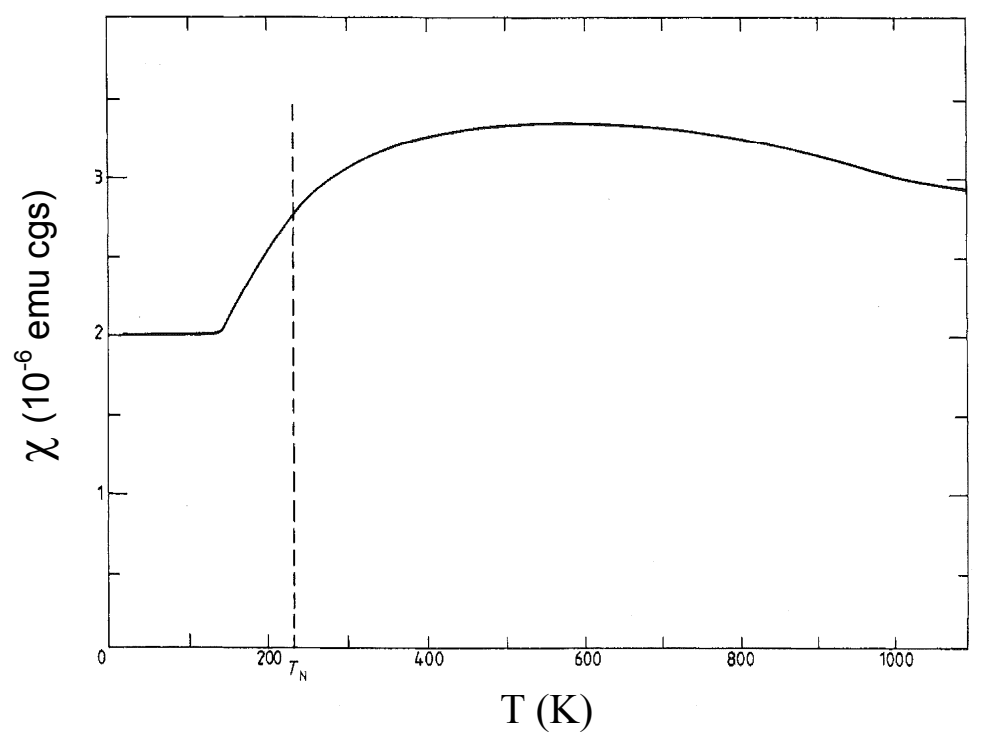

Figure 9. Schematic diagram of magnetic susceptibility of cupric oxide, CuO. Reproduced, with author and publisher permission, from [44]. Copyright 1988 IOP Publishing.

Cupric oxide is an intrinsic p-type semiconductor with application in electronics for its (comparatively) small bandgap. Petrov et al. [45] remark that, "...copper oxide [CuO] is a weakly degenerate semiconductor,...". As a semiconductor doped $\mathrm{CuO}$ has been studied 
for decades. Much of the doping in electronics does of course, occur at temperatures well above those necessary for the superconductivity so far discovered. Even so, the substance is well studied, often doped, and doping $\mathrm{CuO}$ has not been found to result in superconductivity, even though $\mathrm{CuO}$ has an approximately square planar co-ordination as seen in the cuprate HTS. It does not however have the same layered structure, which is clearly vital.

Copper is a transition metal and has a filled $3 \mathrm{~d}$ electron shell. Unlike the other monoxides of the $3 \mathrm{~d}$ transition elements, which are cubic and octahedral, cupric oxide has a monoclinic unit cell [44]

There is also the elemental oxygen, which enters and leaves many cuprates as they are doped through a range of electronic phases and superconducting critical temperatures. This oxygen is present both in molecular form as adsorbed molecules and in atomic form as the labile oxygen in the atomic structure.

\subsection{Oxygen}

As oxygen plays a vital role in the production of superconductivity in the cuprates, it is worth gaining familiarity with properties and behaviour of this element both as it stands alone, and as it interacts with different environments, particularly those of nano-sized structures. It is reasonable to consider the possibility that excess oxygen may find its way inside crystals of the cuprates due to the pressures exerted by the anneal process, most significantly in the extremely over-doped regime. Further, unlike most other HTS Bi- 
2212 has a very "platey" microstructure with a very high internal surface area onto which oxygen can adsorb.

Apart from its ability to rust many of the objects of everyday life, oxygen is renowned for its magnetic properties. Moreover, it undergoes many structural changes with temperature and pressure variation. Nanospace geometries provide a pseudo high pressure environment for confined oxygen. Small spacing within a (crystal) structure can hold inserted molecules to a particular alignment.

Where the molecule or atom is trapped inside the crystals of the host, instead of between them, the combination is called a clathrate. Pure, bulk oxygen without the confinement of an exo-structure already has a rich phase diagram, as for example displayed by Gorelli et al [46]. The $\delta$-phase, like the $\alpha$-phase, is antiferromagnetic. With increasing pressure, both phases may exist to $200 \mathrm{~K}$, and above for the case of the $\delta$-phase. Gorelli et al. [46] note the oxygen is "the only known material that shows a magnetic ordering with increasing temperature", as it warms within the appropriate pressure interval from the diamagnetic $\mathrm{O}_{8} \varepsilon$-phase to the $\mathrm{O}_{2} \delta$-phase where it can exist to room temperature. The $\beta$-phase has short-range magnetic order but lacks the long-range order of antiferromagnetism [47].

The phase diagram changes with restrictions imposed by the presence of a nanostructure. Historically, graphitic nanospace has been the system of choice for the study of oxygen in minute cavities. 


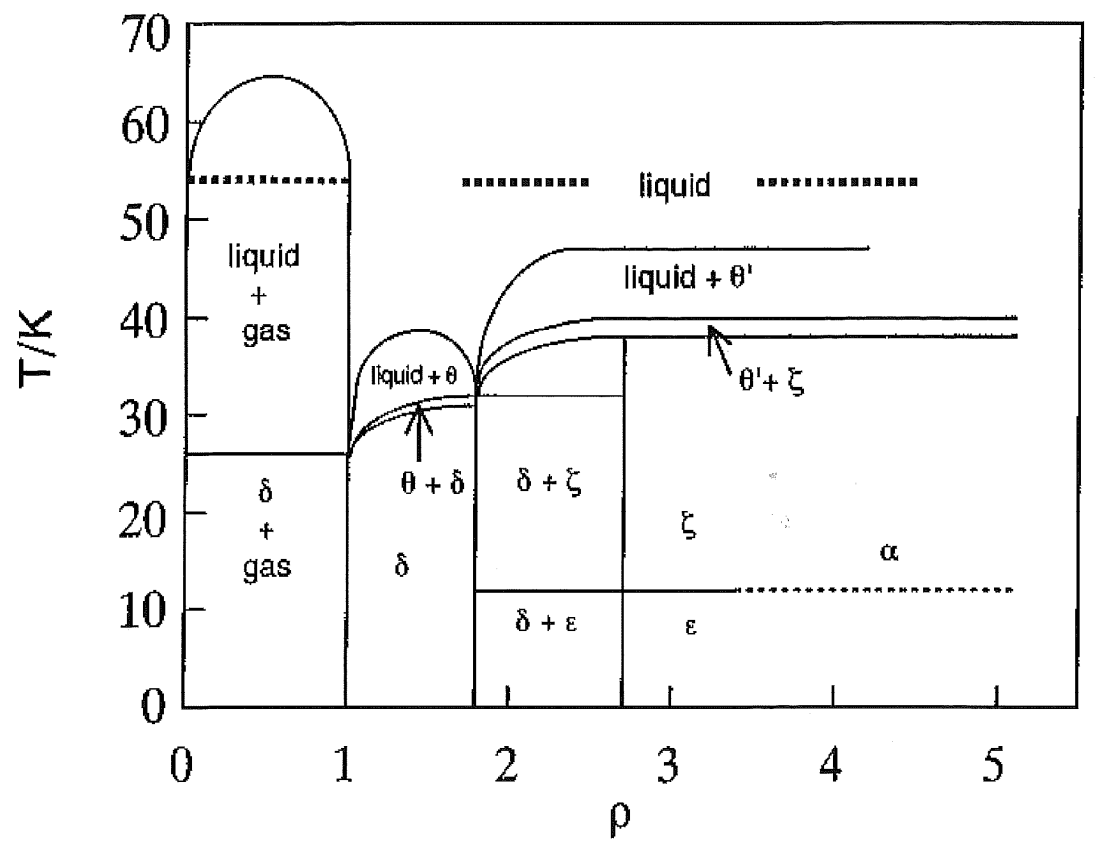

Figure 10. Phase diagram for the $\mathrm{O}_{2}$ /graphite system. Here, $\rho$ is coverage of oxygen, see text. From Kanoh and Kaneko [48]. Reprinted with permission from J. Phys. Chem. 100, 755-759, Hirofumi Kanoh and Katsumi Kaneko. Magnetic Spin States of $\mathrm{O}_{2}$ Confined in a Graphitic Slit-Shaped Nanospace at Low Temperature. Copyright (1996) American Chemical Society

The $\sqrt{3} x \sqrt{3}$ commensurate structure is used to define $\rho$ of Figure 10, where $\rho$ is the coverage of oxygen. See ref. [48] for detail. Measurements were taken over a range of nanospace pore widths (slit pores) for temperatures of 1.7-100K. Note the variation of oxygen phases. Coverage is varied in the same manner as employed in the oxygen doping. Kanoh and Kaneko [48] report increasing antiferromagnetic ordering with increasing coverage. Graphite itself is a 2D layered system.

Kitaura et al. [49] observed the adsorption of oxygen into the channels of $\mathrm{CPL}-1$, a $\mathrm{Cu}(\mathrm{II})$ microporous coordination polymer that 
forms 1D nanospace. Pyrazine (pyz) is used to form the channels between 2D layers composed of the $\mathrm{Cu}(\mathrm{II})$ and pzdc (2,3pyrazinedicarboxylate). The oxygen was inserted by heating the nanospace to remove water molecules and then cooled in oxygen. They measured one molecule of oxygen adsorbed per copper atom, with no electron transfer between gas and solid host. The 1D chain had a ladder structure of parallel oxygen molecules, van der Waals dimers, and a stronger antiferromagnetic interaction than the bulk $\alpha$ phase. At about $90 \mathrm{~K}$ a peak at $1561 \mathrm{~cm}^{-1}$ in the Raman spectra was equated to the solid $\alpha$-phase at $2 \mathrm{GPa}$, rather than to the liquid or gaseous phases. At a pressure of $2 \mathrm{GPa}$ bulk $\alpha$-oxygen exists up to a temperature of around $80 \mathrm{~K}[46]$.

Clathrates offer a form of trapping an incoming molecule or atom within a nanospace where the 'guest' substance is caught inside the crystal of the 'host'. Again, the consequent characteristics impact on the response of the overall system. Apart from degree of chemical interaction with the host a guest molecule in a clathrate may retain ability to rattle e.g. [50], and rotate. Intersecting $\beta$-quinol $\left(\mathrm{C}_{6} \mathrm{H}_{6} \mathrm{O}_{2}\right)$ molecules form a cage that easily traps smaller molecules without chemical interaction, and has been used to study basic effects of clathration. Generalisations, as well as specifics to the nature of the individual gas, are possible. Although the host structure (an organic compound) is - again - not related to the cuprate components, it serves to illustrate the physical effects of clathration.

One notable characteristic is that generally, vibration, rotation and oscillation of the caged gas contribute significantly to overall heat 
capacity [50]. The guest-host interaction of a clathrate affects the thermodynamic stability of the overall system.

Grey and Staveley also refer to the influence of whether or not neighbour and next-nearest neighbour cages are filled. They conclude an increasing potential barrier to rotation as neighbouring cages fill. There are geometrical and chemical (in particular van der Vaals) differences between different hosts, but the study of clathrated (and diatomic) gases finds commonality running through the trapping of the gases inside crystals compared with gases caught between crystals of a polycrystalline sample. In BSCCO the structural location most prone to such intercalation is the weakly bonded $\mathrm{Bi}_{2} \mathrm{O}_{2}$ double layer.

Bandow et al. [51] study the effect of oxygen adsorption on carbon nanohorns, a closed-off version of the carbon nanotube. They note oxygen molecules can get into the interior of the horns through openings caused by the heat treatment (which the nanohorns are subjected to on purpose, for creating entryways for hydrogen storage). By heat treatment they mean temperatures of normally $400-500{ }^{\circ} \mathrm{C}$ in an atmosphere of dry air. This is comparable to the anneal conditions for the BSCCO.

Also available for contemplation is the magnetic susceptibility of bulk oxygen as cooled under constant volume in a closed environment without maintenance of standard pressure. This is similar to a SQUID chamber which, although strictly speaking is not a closed system, mimics the cold, low-pressure scenario via the efforts to maintain a partial vacuum. 


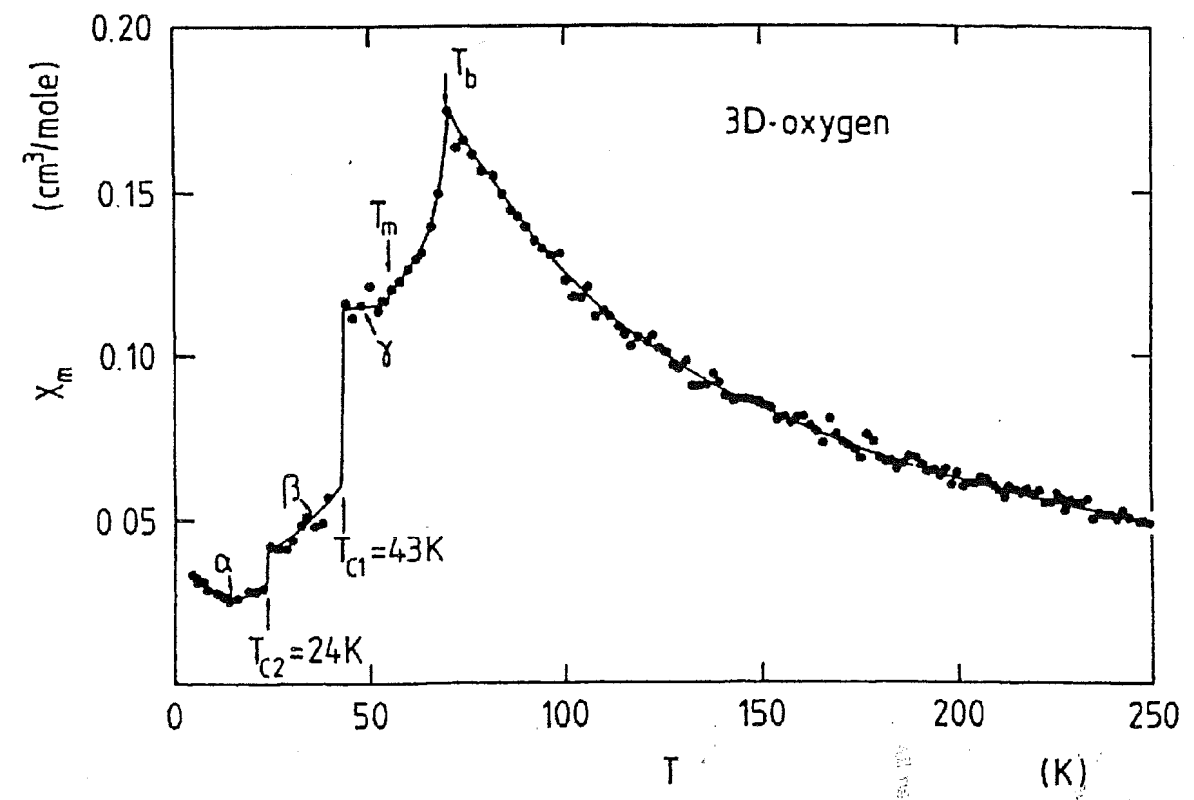

Figure 11. Susceptibility of cooled oxygen in constant volume. From [52].The oxygen was in a small closed quartz cell of unspecified dimensions, but of sufficient size to permit the formation of 3D bulk oxygen configurations. Subsequent surface area and 'slit width' available to the oxygen was controlled by the addition of varying quantities of exfoliated graphite (can be thought of as a variety of activated carbon, produced by vaporisation of the intercalate in the graphite). Reprinted (figure 1) with permission from: U. Köbler and R. Marx, Phys. Rev. B 35, 9809. Copyright 1987 by the American Physical Society. http://link.aps.org/doi/10.1103/PhysRevB.35.9809

The freezing point and the solid $\gamma-\beta$ transition shift little from standard pressure but the boiling temperature drops to $70 \mathrm{~K}$ (from 90K). Oxygen sitting on or near surfaces may experience a similar pressure environment and pass through a similar sequence of phase changes. The shape at $T>70 \mathrm{~K}$ follows almost exactly the shape of the susceptibility curve of the normal state of the BSCCO (an oxygen doped cuprate). Thus, any contribution to the BSCCO magnetisation 
from such a source would merge apparently seamlessly with the BSCCO response.

Köbler and Marx also noted that the location and nature of the specific heat peaks of oxygen can pass through changes as internal coverage increases:
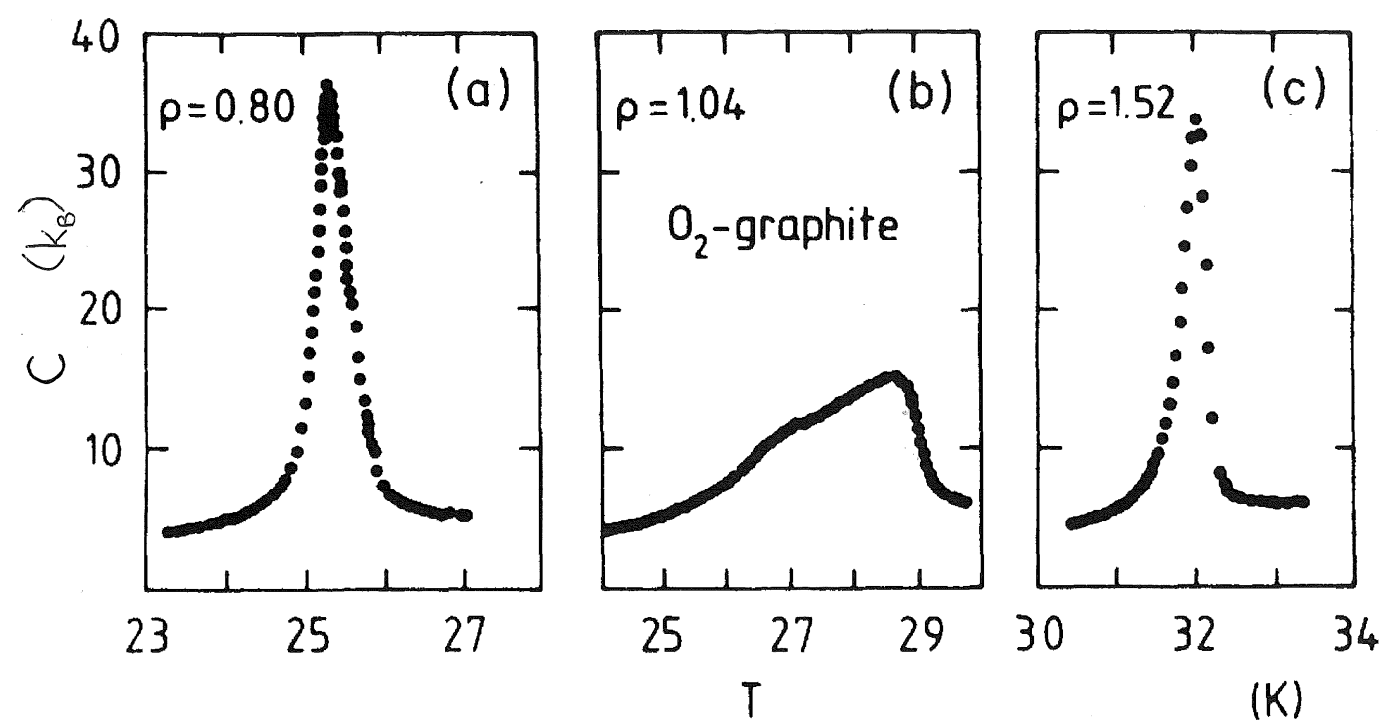

Figure 12. Specific heat of physisorbed oxygen. [52]. Specific heat for some different coverages of 2-d oxygen in graphitic nanospace. In units of $k_{B}$ per particle. They say the flattening and widening at coverage $\rho=1.04$ "reflects a blurred first-order transition from the solid $\delta$ phase to the liquid phase". Refer to Figure 10 for placement of these peaks in the phase diagram. Reprinted (figure 4) with permission from: U. Köbler and R. Marx, Phys. Rev. B 35, 9809. Copyright 1987 by the American Physical Society.

http://link.aps.org/doi/10.1103/PhysRevB.35.9809

The interest in nanotechnology has lead to the development of the porous coordination polymers, starting from the CPL-1, into controllable nanospace [53] categorised by parameters such as: 
size, shape, distribution of cavities; dimensionality, and ability of the host framework to interact chemically with the guest. These will facilitate the systematic study of gases in nanospaces ranging from the purely structural host that does not bond with its guest through to functional hosts where the guest gas is affected chemically along with having the physical constraints.

In the specific case of BSCCO, as oxygen is doped in from the parent Mott compound, the $T_{c}$ increases then decreases. The microstructure of cuprate crystals forms a nanospace geometry. As the first oxygen molecules dope in to the antiferromagnetic parent, it could be that all, or most, of the oxygen is readily accepted by the copper as a doping agent. It would seem feasible that as oxygen doping continues, there could become a point of semi-saturation beyond which the internal oxygen bonds less easily with the cuprate, particularly in the overdoped region. This would be observed in ARPES as a slowing in the rate of change in the Fermi surface area of the copper oxide planes, until no further doping takes place and the Fermi surface freezes.

On reflection it is easy to see that in everyday situations we are accustomed to thinking in terms of force, rather than the pressure achieved. A common level of force, applied to a microscopic entity can be equivalent to enormous pressure (witness diamond anvil cells which with the push of a finger can achieve 3 million atmospheres of pressure). Solid oxygen undergoes interesting phase transformations when subjected to ever higher pressure. Increasing quantities of oxygen inserted into nanospace geometries, such as those provided by layered compounds, undergoes similar 
changes, without the experimenter needing to apply the dozens of GPa necessary to achieve such change for the bulk oxygen. Interfacial energies can result in internal pressures greatly in excess of external pressures.

That the oxygen doping of the cuprates is easily achieved and reversed at temperatures of hundreds of degrees cooler than the melting point of either the cuprate or any of the layers, in an atmosphere of flowing oxygen, implies the introduced oxygen is weakly bound and has a flexibility of behaviour. Whether or not the material is completely free of any physically adsorbed oxygen, bonded oxygen may also show some of the properties discussed above and the whole crystal coordinate as a functional guest-host combination.

Leaving out the ferromagnetic elements iron, cobalt and nickel, and the corrosive fluorine, only nine of the elements with stable isotopes have greater paramagnetic susceptibility than oxygen (ref. data compiled in [54]). At standard temperature and pressure, there is a very large difference between oxygen and the majority of the elements with lesser susceptibility [54].Moreover, paramagnetism increases substantially on cooling, thus markedly increasing the difference between oxygen and the rest at BSCCO superconducting temperatures. Oxygen at room temperature has a magnetic susceptibility of $344910^{-6} \mathrm{~cm}^{3} \mathrm{~mol}^{-1}$ [54], rising to $769910^{-6} \mathrm{~cm}^{3} \mathrm{~mol}^{-1}$ at the liquid state $(90 \mathrm{~K}$.). In these units the (Bi, $\mathrm{Pb})-2212$ response varies around $20010^{-6} \mathrm{~cm}^{3} \mathrm{~mol}^{-1}$, with the exact value dependant on doping state. The difference is great. 
This is the substance the BSCCO is cooked in to achieve superconducting levels of doping.

A literature search did not encounter any examples of previous works that attempt to account for oxygen contribution to the data either by analysis of the oxygen component of magnetic response, or by acknowledgement of any oxygen effect on magnetic data specifically taken to measure the response of an oxygen-doped cuprate. The writer hopes the preceding gives sufficient demonstration of the strength of the magnetic versatility and influence of oxygen under various conditions. At the very least, the $\mathrm{BiO}$ layer in the BSCCO should be studied enough to eliminate the possibility oxygen as a dopant for the cuprates contributes significant magnetic influence from its position external to the superconducting copper oxide layer. It is not sufficient to just assume such a noncopper-oxide magnetic contribution to data to be negligible. 


\section{Experiment}

\subsection{Data acquisition}

Magnetic susceptibility data was acquired by measuring magnetic moment as a function of temperature in a static field. Field-cooled (FC) magnetic data was collected to determine $T_{c}$ to gain an idea of the doping state and its progression with annealing history. Then, normal-state data between $90 \mathrm{~K}$ and $350 \mathrm{~K}$ was measured at $50 \mathrm{kOe}$ under zero-field cooling (ZFC), where the sample is cooled under zero applied magnetic field and then the field is switched on and measurements taken on warming. The purpose of this was to try to put upper and lower bounds on the VHS location. Earlier, when the presence of the $\mathrm{vHs}$ had been confirmed in the magnetic susceptibility, the doping state had been assessed through $T_{c}$ values obtained from ZFC data. Although sufficient to verify the location of the singularity, it was disappointing the level of uncertainty in the measurement of $T_{c}$. The only difference is the method used to locate $\mathrm{T}_{\mathrm{c}}$. High-field data that shows the VHS continues to be taken by the same method. Quenching or not quenching in liquid nitrogen after anneals had not been found to affect results (n.b. anneal temperatures are lower than $400^{\circ} \mathrm{C}$ ).

Measurement of $T_{c}$ for the single crystal and the control polycrystal were taken ZFC to be able to directly compare with the previously known locations of features of the polycrystal. The control polycrystal was annealed with the single crystal and was from the same BSCCO preparation as the main polycrystal. 


\subsection{Doping method}

All samples were doped by being cooked in a flow of pure oxygen at atmospheric pressure. The changes in doping state to pass through the VHS were achieved by altering the temperature for different anneals.

Ideally, one might use a hot isostatic pressing (HIP) furnace to dope high oxygen levels. In the absence of a HIP furnace all our anneals were done in conventional furnaces.

Extreme over-doping to $T_{c}<54 \mathrm{~K}$ is easily achieved in the polycrystal $(\mathrm{Bi}, \mathrm{Pb})-2212$ without the use of high pressure. An early attempt to over dope the single crystal $(\mathrm{Bi}, \mathrm{Pb})-2212$ in a regular bomb furnace by applying $40 \mathrm{~b} \mathrm{O}_{2}$ appeared to be the reason for the shattering of the crystal into shards, perhaps by rapidly varying pressure along the surface of the samples due to the gas flowing through. Moreover, the attempt to load in oxygen had resulted in minimal movement of $T_{c}$, indicative of much further time necessary under the pressure to be able to reach a $T_{c}$ on the overdoped side of the $\mathrm{vHs}$. It appeared undesirable to submit the samples to further dynamic high pressure in case resulting destruction reduced the shards to unusable size. As a consequence, to learn how to achieve extreme overdoping of a single crystal using conventional furnaces took a significant quantity of available time.

As with measurements on polycrystals, the magnetisation of the $\mathrm{Bi}$ 2212 single crystal appeared as a strong signal and the SQUID had 
no trouble detecting a signal from samples of a few milligrams in size. Nevertheless, the SQUID did have problems detecting shards approaching a line, and a better response was obtained from $5 \mathrm{mg}$ in 2D square shape than from a $25 \mathrm{mg}$ long strand, for example.

\subsection{Equipment - SQUID}

Empirical magnetic data for this project was centred on the use of the Magnetic Property Measuring System (MPMS) commercially produced by the Quantum Design company, colloquially known as the SQUID. Magnetic fields to ${ }^{ \pm} 7$ Tesla can be generated, and sample response measured over a temperature range of $2-400 \mathrm{~K}$. It is the same apparatus as used previously for detection of the VHS in (Bi, $\mathrm{Pb})-2212$.

The SQUID measures the bulk (macroscopic) moment, the sum of all magnetic responses within the range of the scan. For example, less than one thousandth mole of manganese(III) oxide, $\mathrm{Mn}_{2} \mathrm{O}_{3}$ $\left(\chi_{\text {molar }}={ }^{+} 1410010^{-6} \mathrm{~cm}^{3} \mathrm{~mol}^{-1}\right)$ will overwhelm a whole mole of diamagnetic copper metal $\left(-5.4610^{-6} \mathrm{~cm}^{3} \mathrm{~mol}^{-1}\right)$ to give a paramagnetic reading. One mole of the famously diamagnetic bismuth contaminated with 0.01 mole of europium would also register as having a paramagnetic moment.

These examples are simple pairings to give a demonstration of results that can be obtained from real life bulk measurements where all possible contributions should be considered. 
Changes to intrinsic behaviour can also occur thorough the natural interaction of the sample with the atmosphere in which it exists. The paramagnetic magnesium $\left({ }^{+} 13.110^{-6} \mathrm{~cm}^{3} \mathrm{~mol}^{-1}\right)$ oxidizes to diamagnetic $\mathrm{MgO}\left({ }^{-10} 10.210^{-6} \mathrm{~cm}^{3} \mathrm{~mol}^{-1}\right)$. Conversely, very little of oxygen-containing atmosphere in with the commonly diamagnetic gases, including nitrogen, chlorine, carbon dioxide, fluorides, helium and the other noble gases, is needed for the total gas to provide strongly paramagnetic data. In fact, oxygen adsorbed into solids, or mixed into liquid phases, can be plainly visible in a SQUID measurement.

\subsection{Mounting of the samples}

It was soon discovered that, as previously experienced with the polycrystal, the single crystal BSCCO gave a strong response to the application of a magnetic field. However, due to the brittle nature of the single crystal formation ("fat" film) it was necessary to work with samples of considerably less mass (small, but not weak signal) and concession had to be made to their fragility.

Polycrystal samples are mounted using two straws. One straw is threaded into the other. The sample is held in place halfway down the tube by the pressure between the inner and outer straws. The tip of the outer straw is then attached to the end of the SQUID rod. Both straws are used full length and this provides a constant background of a good sample holder.

The first trials to measure the response of the single crystal used a gelatine capsule stuffed with Teflon ${ }^{\circledR}$ supported by two lengths of 
inner straw, one at either end of the capsule. Figure 13 shows a typical response.

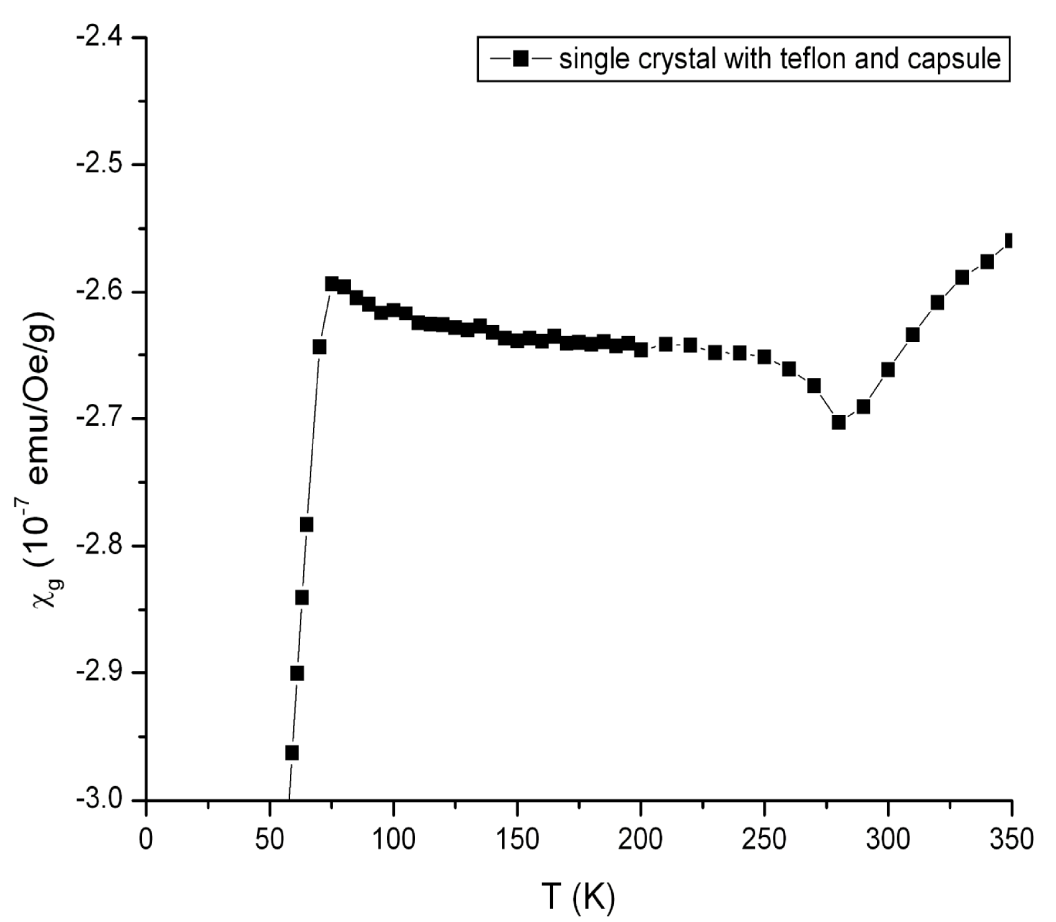

Figure 13. Single crystal in Teflon $^{\circledR}$, gelatine and straw. The result at an applied field of $50 \mathrm{kOe}$ when the single crystal was mounted in a gelatine capsule padded with Teflon with the capsule held in place by straw.

Note the extreme diamagnetism of the system. The BSCCOs are paramagnetic in the normal state. In this case at temperatures above $70 \mathrm{~K}$. There is also extensive distortion of the classic BSCCO form the situation is not simply that of a very small signal on a background of strong diamagnetism which is usually close to constant over a wide temperature range anyway. 


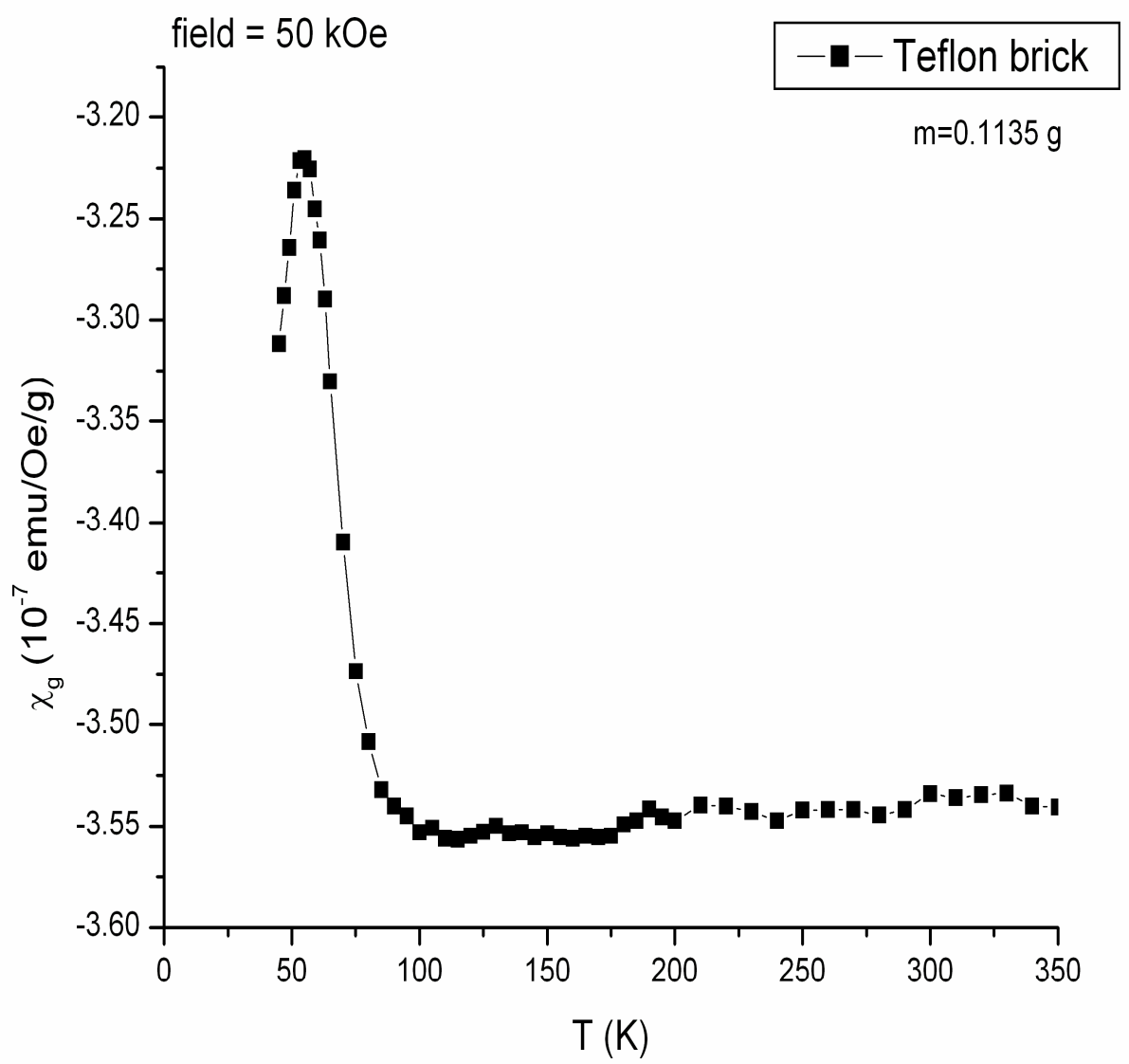

Figure 14. Teflon ${ }^{\circledR}$ magnetic susceptibility. A brick made of folded Teflon ${ }^{\circledR}$ tape and mounted in the same manner as polycrystal samples. Straw layout is uniform along length of measurement. Note the atmospheric oxygen contamination evidenced by the large peak round $54 \mathrm{~K}$.

The Teflon ${ }^{\circledR}$ proved to be more diamagnetic than polycrystalline bricks of BSCCO had been found to be paramagnetic, as can be seen by comparing Figures 1 and 14 . Teflon ${ }^{\circledR}$ also traps atmospheric oxygen with ease, demonstrated by the rising susceptibility below $100 \mathrm{~K}$ with a very high peak at the liquid-solid transition. Note the size of the oxygen addition, which arises solely from trapped molecules from the atmosphere. 


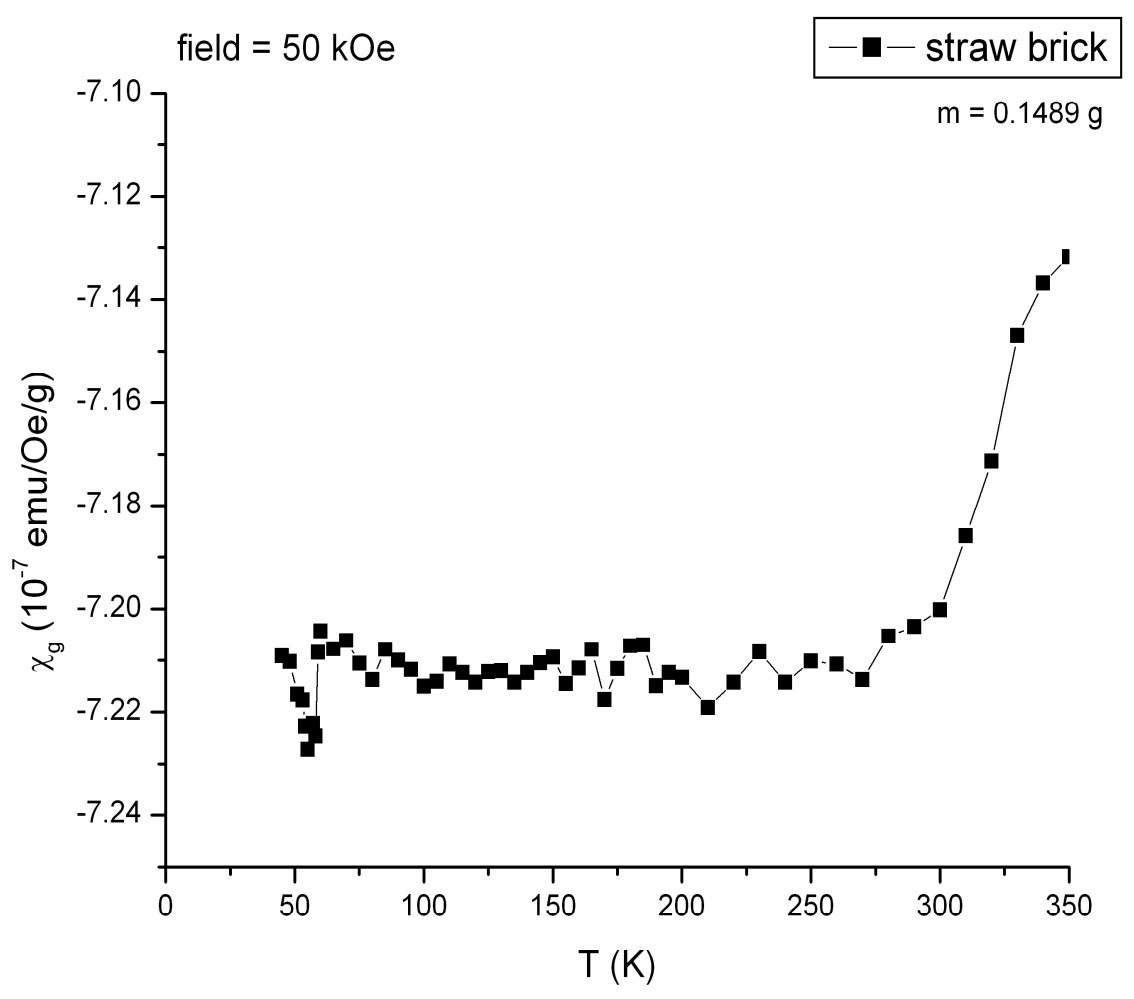

Figure 15. Plastic straw magnetic susceptibility. A straw brick measured under the same conditions as the Teflon ${ }^{\circledR}$ brick and BSCCO polycrystal samples. The inverted spike here is also from the $54 \mathrm{~K}$ magnetic transition of oxygen.

Plastic straw was also measured as a substance in its own right. Slight oxygen contamination in the straw brick was not a surprise as the brick was constructed by layering together rectangles of the plastic and there is little escape possibility for trapped gas. However, the high tail at the high temperature end, as seen in Figure 15, is uncharacteristic of diamagnetic materials, though does match the feature in the combined materials mounted with the single crystal. This feature disappears as the quantity of localised extra straw mass reduces. In the capsule arrangement this tail could easily come from the support straws on either side of the capsule that do not cover the 
site of the sample. Thus the quantity of straw changes dramatically with location.

In practice the response recorded with Teflon ${ }^{\circledR}$ had fascinating and reproducible distortions, in spite of very different mounting arrangements, that disappeared with the removal of the Teflon ${ }^{\circledR}$. On noting the observations and experiences of earlier work on Teflon ${ }^{\circledR}$, e.g. [55] and [56], where response varied essentially with reaction to mechanical stresses, it was realised the extent of the difficulties of being able to accurately account for the effect of the Teflon ${ }^{\circledR}$ for each temperature and extract the true response due to the single crystal. Moreover, the presence of the BSCCO mass appeared to increase the contribution of the Teflon ${ }^{\circledR}$.

A straw-only mount was ultimately found to make the best available sample holder for the single crystals, just as for the polycrystals, with the single crystals able to remain in fixed position just as well as the polycrystals. All samples were mounted in the same manner, with two straws used full length which provides a constant background over the length of the scan.

\subsection{Main Polycrystal}

Obtaining many data points through the location of the VHS is difficult to achieve. The narrowness of the VHS peak requires steps in doping of the order of 0.0003 holes per $\mathrm{Cu}$. This translates to changes in $T_{c}$ of a fraction of a degree to be able to show the up and down progression. Such small steps in doping state require minute changes in the anneal temperatures, which are difficult to control at 
that level due to such real-life effects as fluctuations in power supply, differences between individual thermocouples of the same material, whether or not other equipment in the room is running, and other mundane events.

Acquisition of a number of data points through the peak was achieved by passing through the VHS a number of times by reannealing of the sample. The very small increase in anneal temperature that corresponds to an appropriate step in doping for the VHS is less than the everyday fluctuations occurring in a working furnace with cold gas being passed through it. The gas flow through the oven is turbulent and each anneal gives a different pressure as a function of time experience. Temperature also fluctuates over time differently for separate anneals. So a variety of data with closely spaced $T_{C}$ is achieved by repeating a sample through sequences of very similar anneal conditions to access the small shifts in doping caused by the small-scale changes in pressure and temperature combinations.

In common with other experiments [3], uncertainty in measurement of $T_{C}$ remains large. Strictly speaking the uncertainty in $T_{C}$ is larger than the width of the VHS and is accentuated by the need to reanneal through the VHS a number of times.

Figure 16 shows the shape of the typical BSCCO normal state magnetic susceptibility from a selection of measurements. Referring back to Figure 7 the prediction is that curves near the antibonding VHS will be higher than those far from this VHS. From the theory (equation (1)) the rise to the bonding VHS is not expected to be 
detectable until a doping level of around $p=0.26$, or after $T_{c}$ drops to less than $17 \mathrm{~K}$.

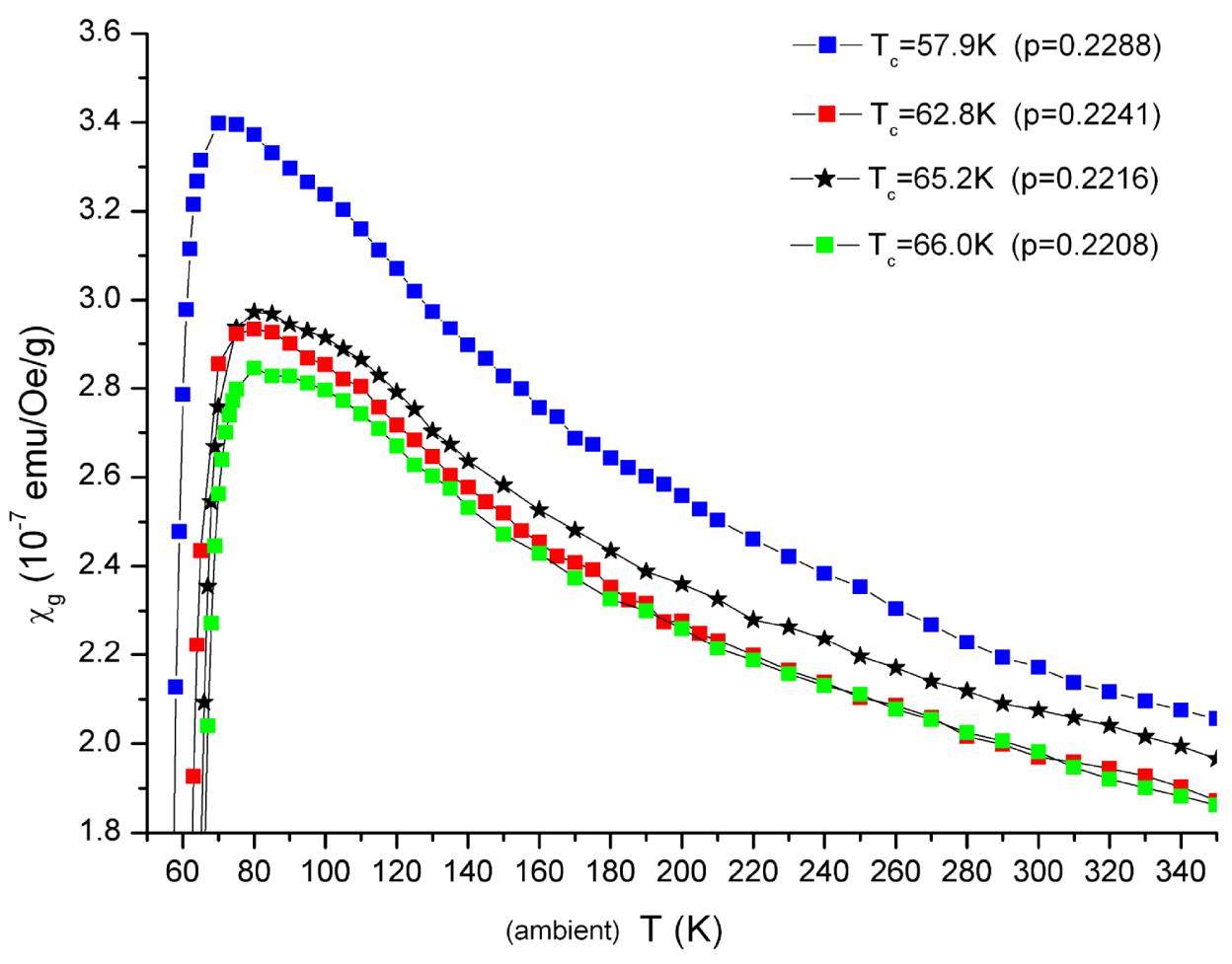

Figure 16. (Bi,Pb)-2212 polycrystal examples of magnetic susceptibility versus temperature. The data curve marked with stars is the one closest to the peak of the VHS for this selection of $T_{c} s$. Only a limited number of data curves are shown.

In this work $\mathrm{T}_{\mathrm{C}}$ is measured under low field (typically $10 \mathrm{Oe}$ ) and is taken as the intersection of the tangents of the normal-state susceptibility above $T_{C}$ and of the maximum slope of the diamagnetic susceptibility below $T_{c}$. For conversion between $T_{c}$ and corresponding doping level, $p$, the standard relation [57] is used.

$$
T_{c}=T_{\max }\left[1-82.6(p-0.16)^{2}\right]
$$

$\mathrm{T}_{\text {max }}$ for the $\mathrm{Bi}-2212$ is $95 \mathrm{~K}$. 
Figure 17 converts the data to vertical slices through constant ambient temperatures presented as a function of decreasing $T_{c} \mathrm{~S}$, as in the overdoped region this corresponds to increasing doping. As previously we see the VHS peak and an increase in the magnitude as doping increases.

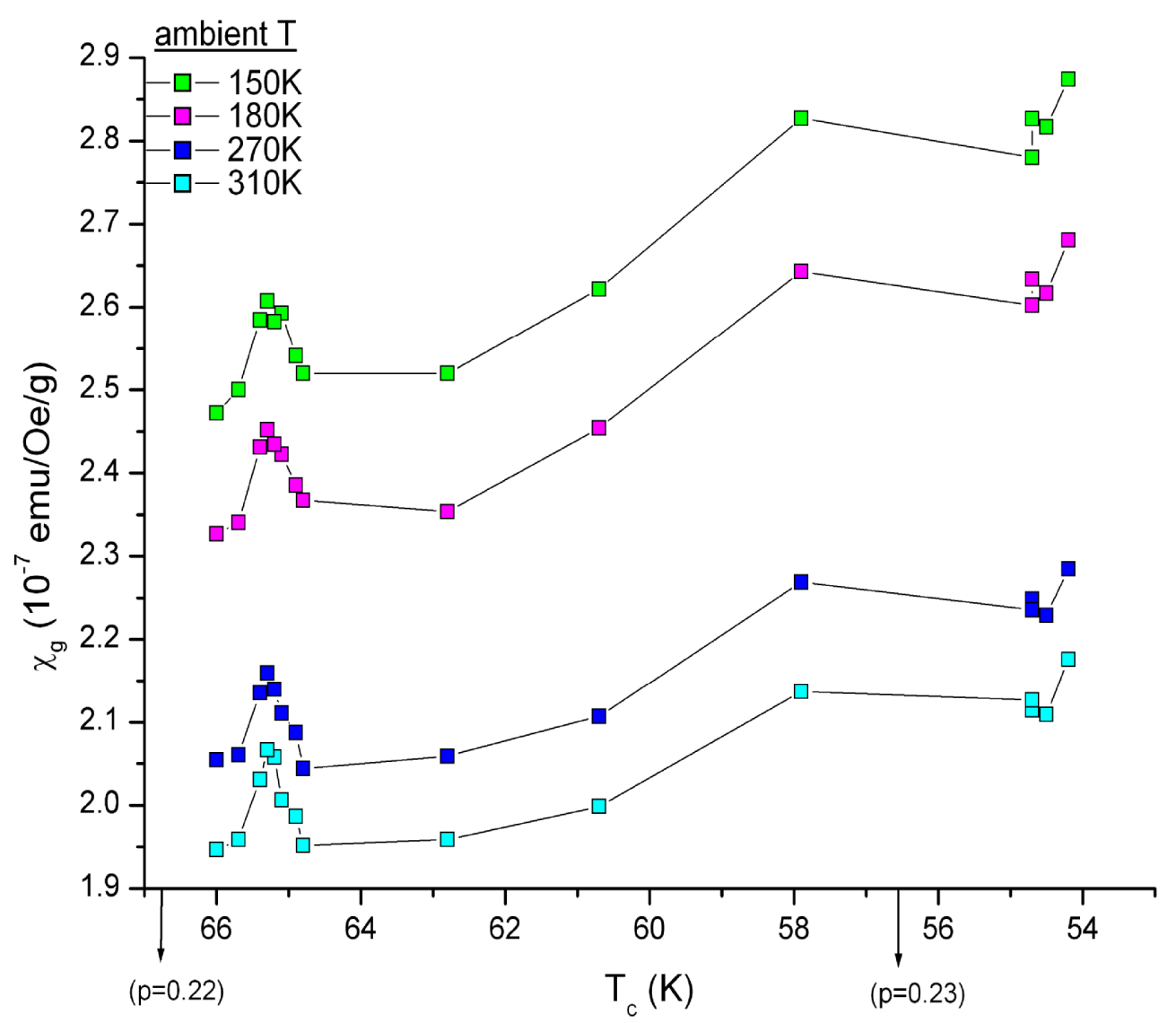

Figure 17. (Bi,Pb)-2212 polycrystal magnetic susceptibility versus $T_{c}$ showing the VHS over a wide range of ambient temperatures. Aside from the peak at the VHS all curves also show a growing increase in magnitude with increasing doping. 
Notice the experimental increase in the magnitude with doping is greater at the colder ambient temperatures. At 270K (Figure 17) the rise from the foot of the VHS to doping of $p=0.23$ is around $0.2 \times 10^{-7} \mathrm{emu} / \mathrm{Oe} / \mathrm{g}$. At $90 \mathrm{~K}$ (Figure 18) the rise over the same interval approximately doubles to around $0.4 \times 10^{-7} \mathrm{emu} / \mathrm{Oe} / \mathrm{g}$. This implies the growing contribution to the susceptibility with doping comes from a paramagnetic source. Also noticeable is the appearance of the VHS peak at $T_{c}=65 \mathrm{~K}$ instead of $T_{c}=61 \mathrm{~K}$, a difference that appears to arise out of differences in measurement method, as subsequent measurements on a sample from the same pellet showed the VHS location returned to $T_{c}=61 \mathrm{~K}$ when the method reverted to ZFC conditions instead of FC (see Figure 30).

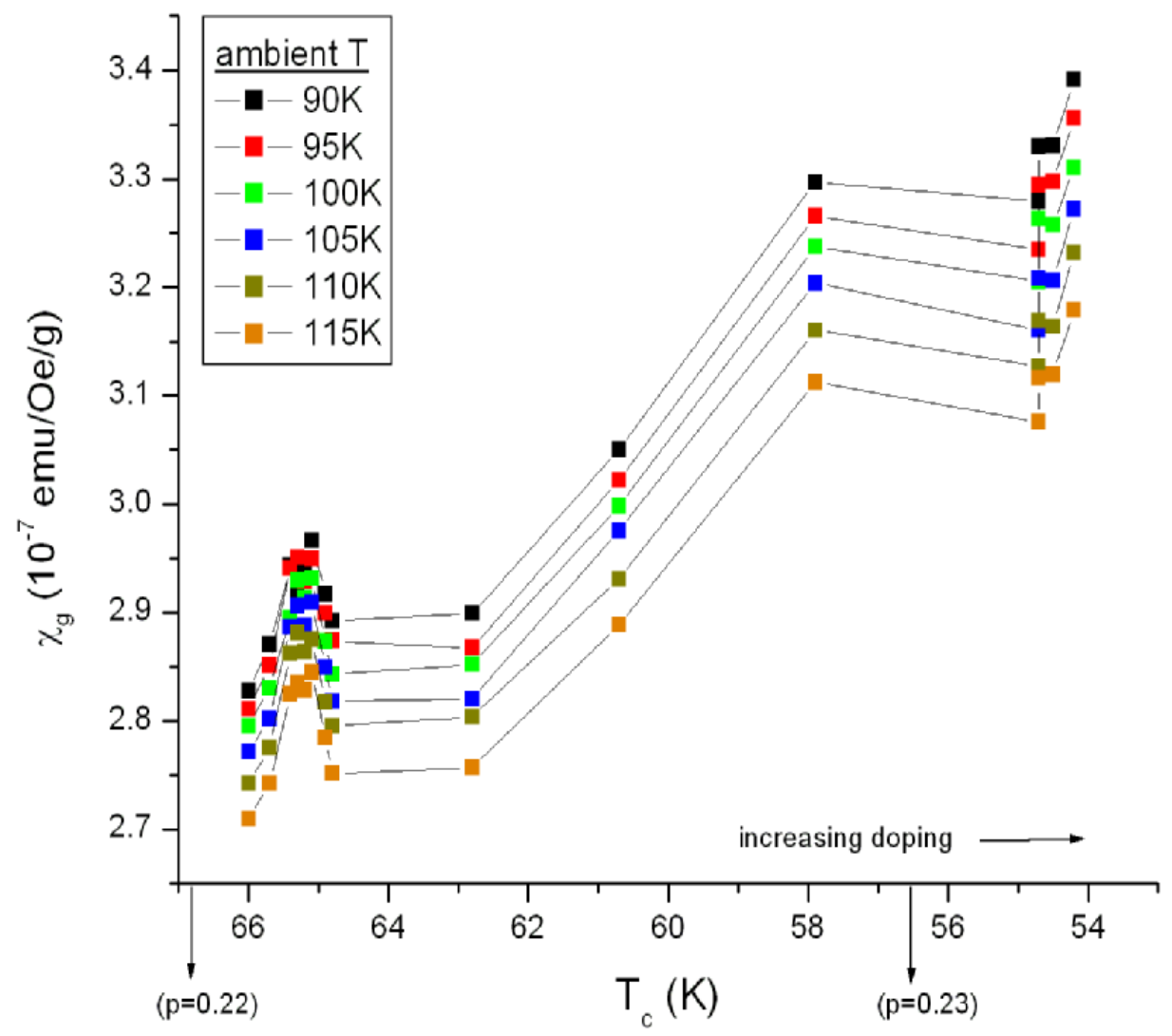

Figure 18. (Bi, $\mathrm{Pb})-2212$ polycrystal VHS for low temperature isotherms. 
Magnetic susceptibility also clearly shows the VHS at isotherms up to, and past, room temperature. This has been a duplicable result. Theory shows diminishing visibility of the VHS with increasing temperature. This reflects the view that the Fermi window only sees the VHS at low temperature, whereas at high temperature it looks out beyond the VHS where the DOS is diminished. Figures 19 and 20 show the theoretical calculations for the magnetic susceptibility magnified from Figure 7 to cover the same doping range as the experimental work.

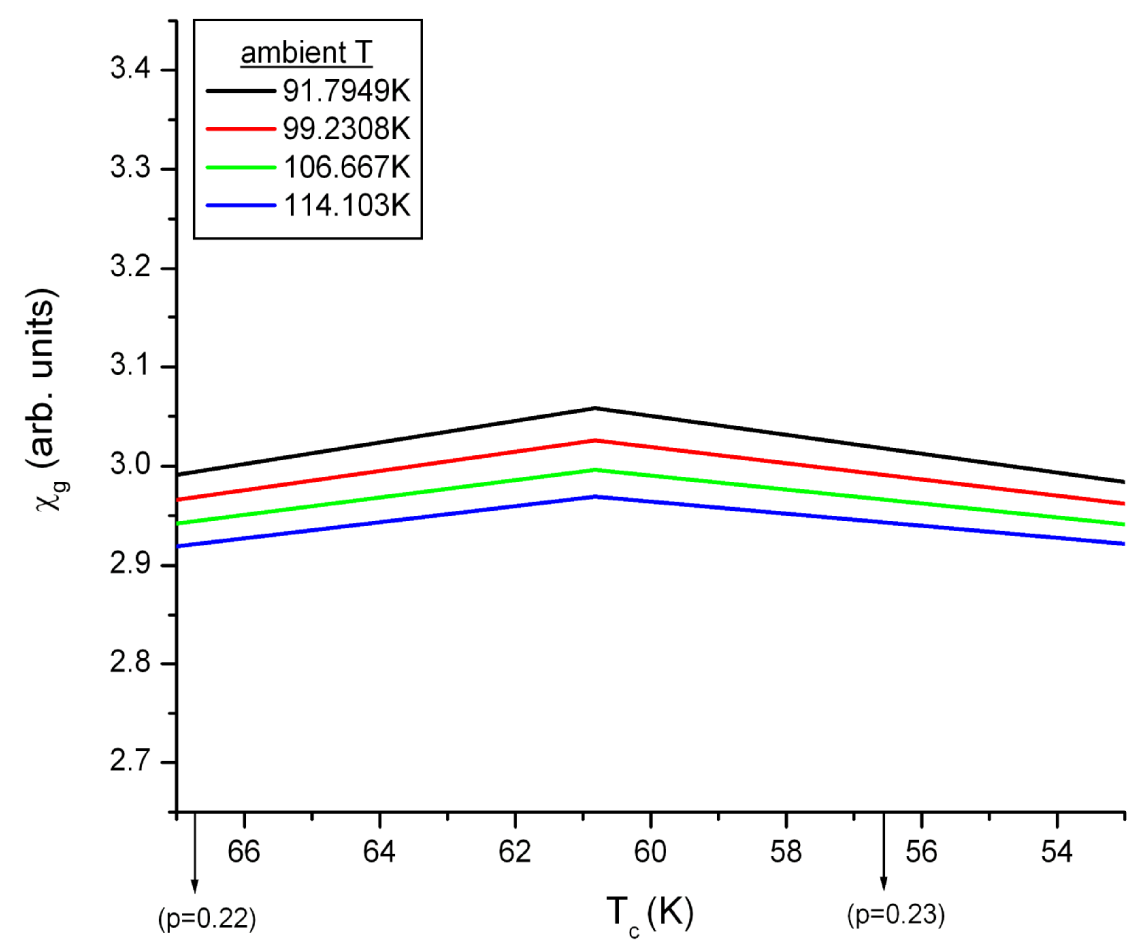

Figure 19. Theoretical calculations for isotherms in the range $90 \mathrm{~K}-115 \mathrm{~K}$ magnified to the same doping range as the experiment. Data from [28], new graph created with permission. The theoretical VHS peak is more noticeable at these temperatures. Notice the susceptibility magnitude falls on both sides of the VHS over the doping range. The bonding VHS (calculated to be at around $p=0.3$ ) is not yet detectable. 


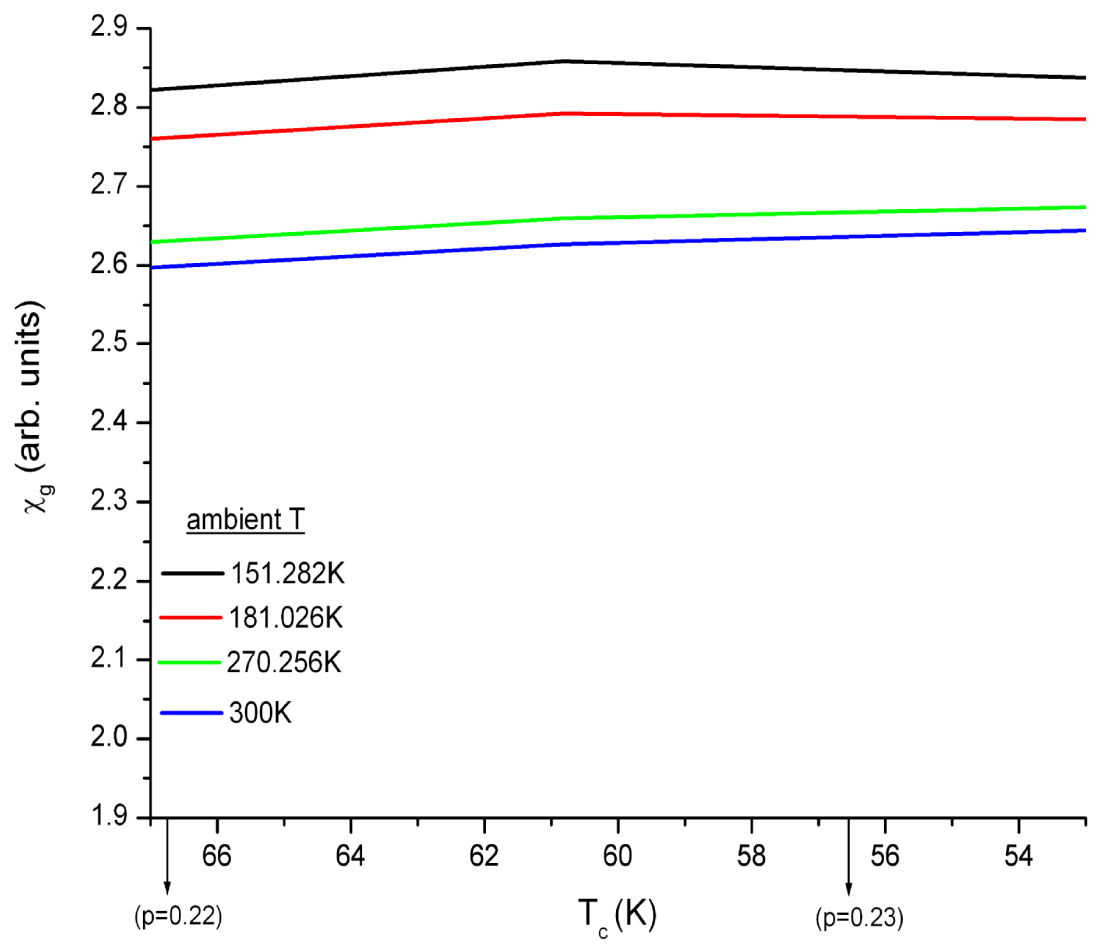

Figure 20. The theoretical calculations for magnetic susceptibility for isotherms within the range $150 \mathrm{~K}-310 \mathrm{~K}$. As the ambient temperature warms the susceptibility magnitude flattens out and visibility of the VHS is minimal compared to how noticeable the peak is in Figure 17. Data from [28], new graph created with permission

In this experiment $T_{c}$ was evaluated for the polycrystal using $\mathrm{FC}$ instead of the ZFC of the previous experiment to try to use the difference between the two to put upper and lower bounds on the location of the VHS. It was completely unanticipated to find a difference as large as four degrees for locating $T_{c}$. This is much too large a shift to be accounted for by the 10 Oe field used. If this large difference had been known about at the start, the $T_{c}$ measurement time would have been used to do dc only for both ZFC and FC placements of the $T_{c}$ on the same sample at the same doping level, instead of taking ac as well as dc measurements for FC only. The ac data is slightly noisier FC than previously for ZFC, but does not 
contradict the dc data. Data from the control sample demonstrated the VHS peak at $65 \mathrm{~K} \mathrm{FC}$ is equivalent to a peak at $61 \mathrm{~K} \mathrm{ZFC} \mathrm{(see}$ Figure 30).

From ARPES measurements Kaminski et al [3], detected the VHS crossing in the doping range of $p=0.22$ to $p=0.23$ (or, somewhere between $T_{c} s$ of $57 \mathrm{~K}$ and $67 \mathrm{~K}$ ).

As with ZFC, the rate of change of temperature FC was one degree per minute plus time during which the temperature is held constant while the magnetic response is measured. As the VHS normal state data itself is measured the same way in all cases (applied field of 50 kOe ZFC), one could expect that the anomalous peak previously measured at $58.9 \mathrm{~K}$ be now visible at $58.9+4=62.9 \mathrm{~K}$. The data point very near $62.9 \mathrm{~K}$ shows no lift away from the background.
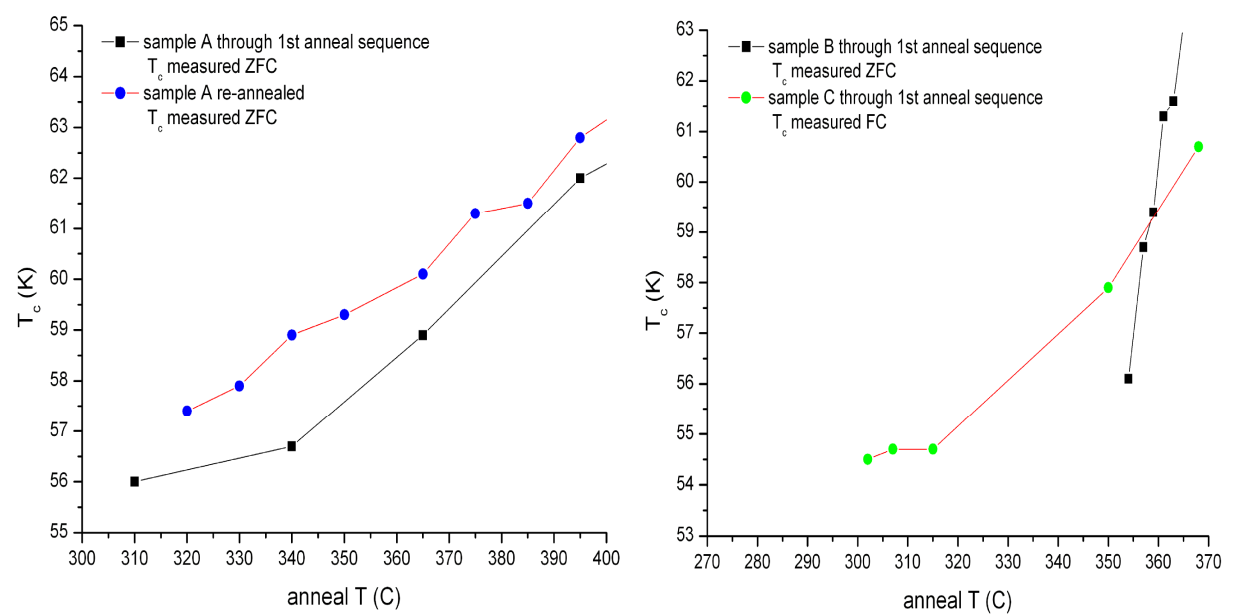

Figure 21. Effects of reannealing and of measuring ZFC or FC. Sample A shows the typical shift in $T_{c}$ that occurs when a sample is repeated through a sequence of anneal conditions. Samples B and C, from the same ceramic block, have only been through one sequence of anneal conditions each. $T_{c}$ was measured differently. The atmosphere for all three samples is $1 \mathrm{~atm}$ of flowing oxygen. 
It is noted the ZFC and FC both tend toward showing $T_{c} \sim 59 \mathrm{~K}$ for a particular anneal. For $T_{c} s$ lower than $59 \mathrm{~K}$, a $T_{c}$ measured $Z F C$ is increasingly less than the $T_{c}$ measured by $F C$. Above $59 \mathrm{~K}$ the relative difference switches and the ZFC measured $T_{c}$ becomes increasingly greater than that shown by a FC measurement, see Figure 21 for the interchange.

The VHS is not a delicate phenomenon. Regardless of the zigzagging back and forth through doping levels the VHS remains clearly visible and in fixed location. Considering the $2 \mathrm{D}$ nature of the cuprates, it would actually be quite exotic physics if there were no VHS. But: if one did not know where to look, it could easily be missed.

Relative scarcity of data between $T_{c}$ of $58 \mathrm{~K}$ and $64 \mathrm{~K}$ is due to mishaps in annealing that can result from the need to change doping levels by very small quantities. Even a change of oven can disturb the anneal process. The gas flow, and therefore the pressures, may change. A different thermocouple can show the same reading for a slightly different temperature. If a subsequent anneal is in reality a little cooler than the previous anneal, this restarts oxygen uptake. The doping state shifts, perhaps backward as well as forward. The magnitude of the susceptibility is also disturbed as loading oxygen into a sample takes much longer than removing it.

HTS practice historically subtracts a Curie-Weiss term to deduct background contributions (from e.g. magnetic impurity phases such as $\mathrm{CuO}, \mathrm{SrCuO}_{2}$ ) and considers the remainder the response from the superconducting layer. This needs reassessment. Triscone's 
polycrystal data (see Figure 23) does subtract a Curie-Weiss term, and still has the monotonic increase in magnetic susceptibility with increasing doping. Cuprate data are traditionally attributed exclusively to the copper oxide planes.

In comparing the data of our polycrystal with the Triscone polycrystal note that each experiment was over a different range of dopings. As well as the monotonic increase in the magnitude of the magnetic susceptibility that accompanies increasing doping, the graphs accentuate the very limited range over which the VHS is visible.

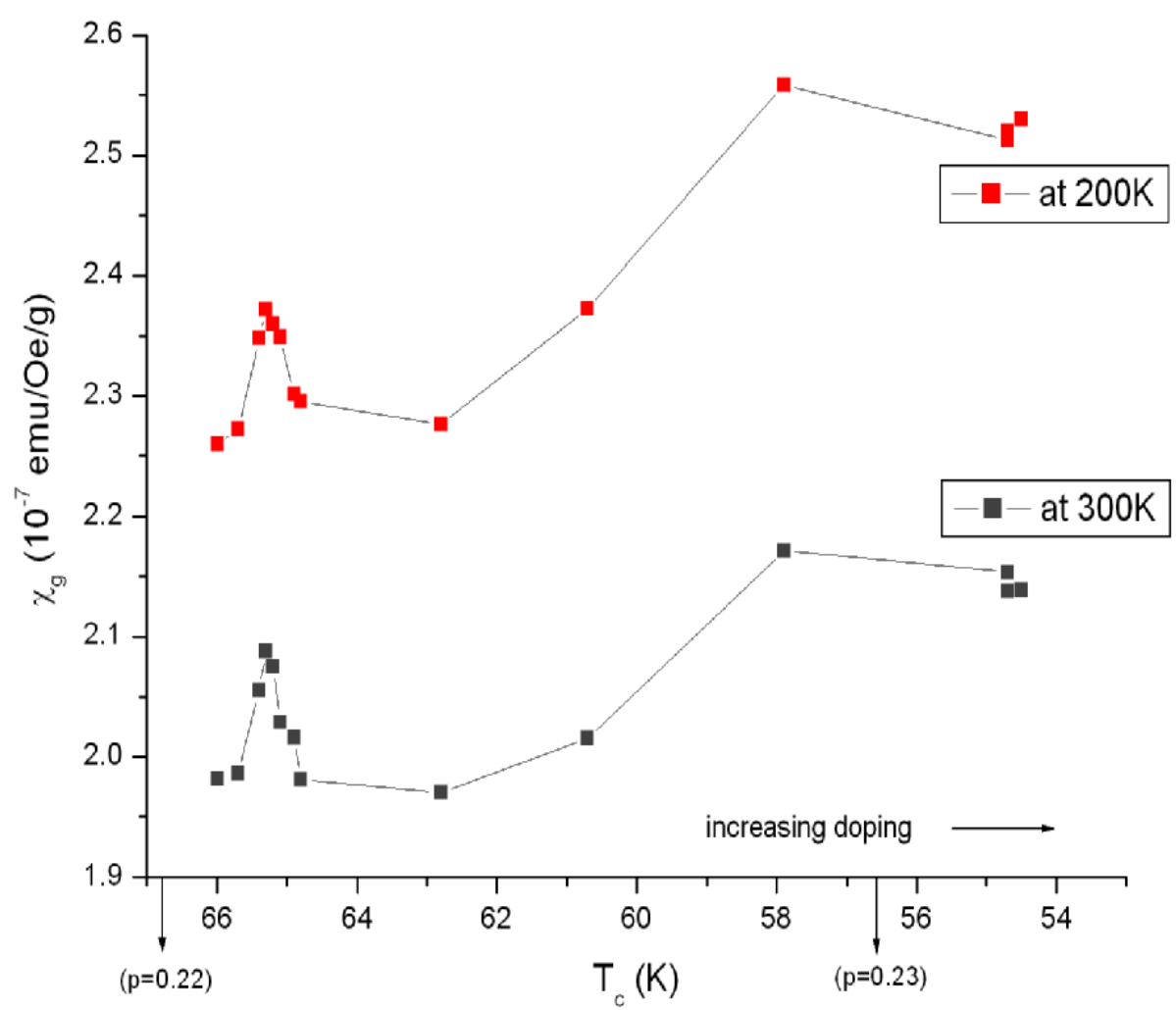

Figure 22. Our polycrystal data for $200 \mathrm{~K}$ and $300 \mathrm{~K}$ isotherms. If we had measured $\mathrm{T}_{\mathrm{c}} \mathrm{ZFC}$ the VHS would be shifted $4 \mathrm{~K}$ to the right (towards the higher doping end) and appear at $\mathrm{T}_{\mathrm{c}}=61 \mathrm{~K}$ 


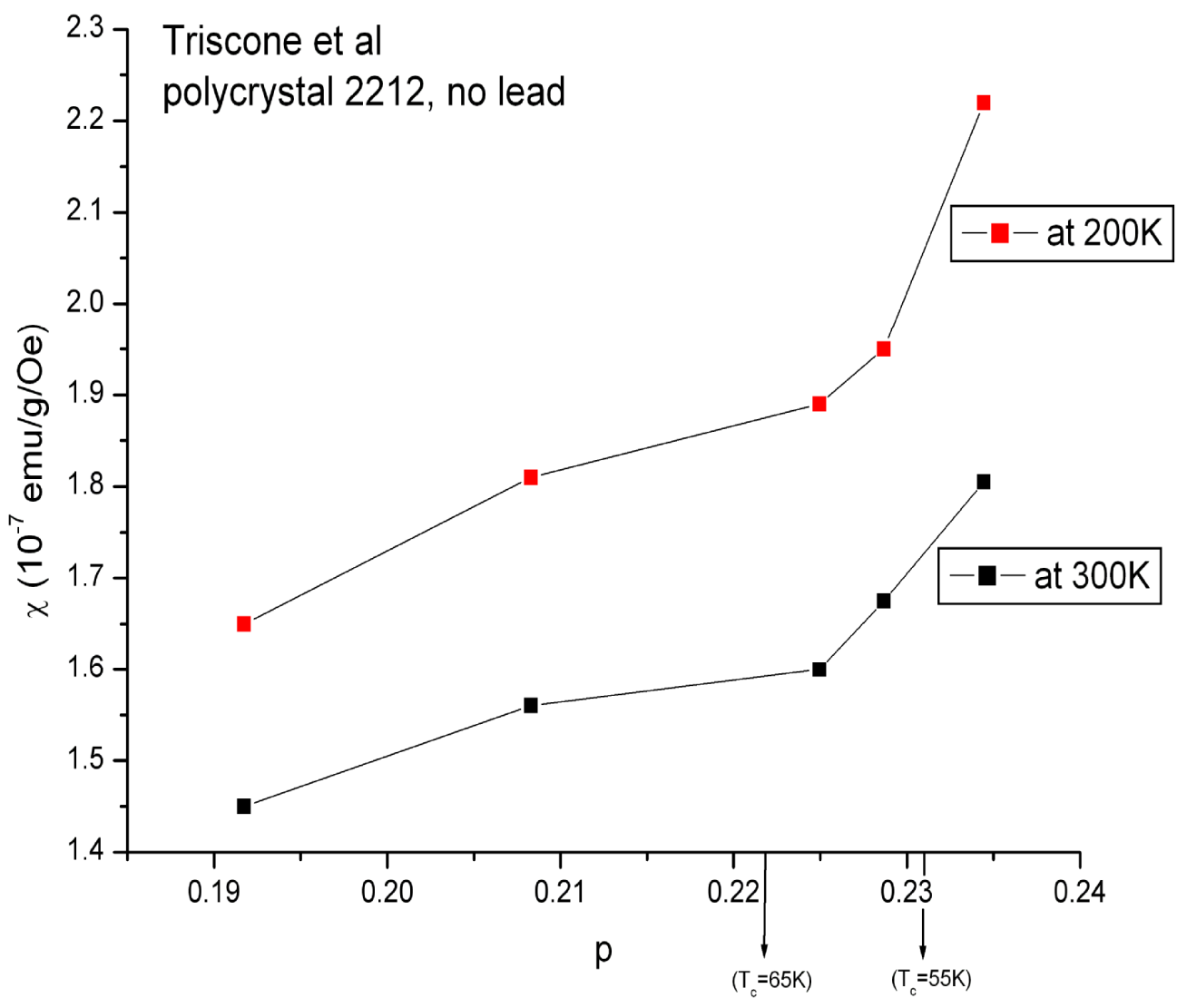

Figure 23. Triscone et al.polycrystal data for isotherms $200 \mathrm{~K}$ and $300 \mathrm{~K}$. Note where the range of our experimental data falls. Vertical view of $\mathrm{Bi}-2212$ magnetic susceptibility [4]. Reprinted from Physica C. 176/1-3, G. Triscone, J.-Y. Genoud, $\mathrm{T}$. Graf and J. Muller, Variation of the superconducting properties of $\mathrm{Bi}_{2} \mathrm{Sr}_{2} \mathrm{CaCu}_{2} \mathrm{O}_{8+\delta}$ with oxygen content, pages 247-256. Copyright 1991 with permission from Elsevier

On the other hand $\mathrm{La}_{2-x} \mathrm{Sr}_{x} \mathrm{CuO}_{4}$, which is not doped with oxygen, does not exhibit a monotonic increase in susceptibility magnitude with doping. It has a VHS at a predicted doping of $x=0.25$, e.g. [58].The magnetic susceptibility rises as a function of approach to this doping level. As the LSCO progresses past this point further into the overdoped regime the magnitude of the susceptibility falls off 
again, as is consistent with a VHS. The disappearance of superconductivity at the edge of the superconducting zone is very close to the VHS in this material.

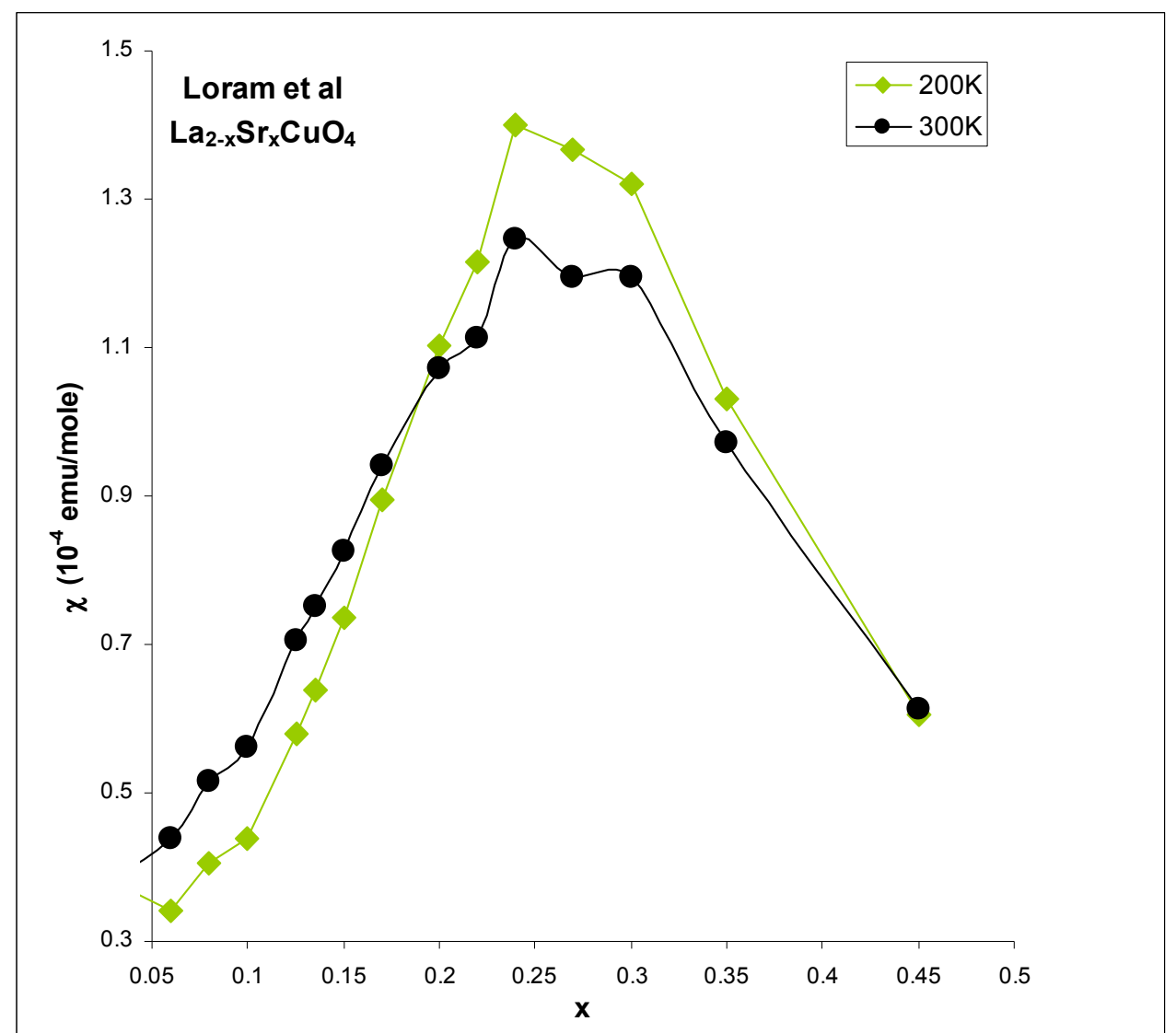

Figure 24. The VHS in the magnetic susceptibility isotherms for the single layer cuprate LSCO. Thanks to the authors and publisher of [59] for use of the data.

On the matter of our background not having been subtracted, as is more usual for presentation - we believe significant background to come from the oxygen. It is uncertain how to appropriately account for background that comes with the crystal itself (and therefore absent when the crystal is out of the chamber during the more conventional means of attempts to measure background). Moreover, 
magnetic susceptibility background from the oxygen seems likely to include contribution from oxygen that has both increased the total paramagnetism and at the same time bonded sufficiently to the host cuprate to donate holes. Apart from the fact that it is not yet known how to magnetically differentiate the charge donor and acceptor planes, no precedent has been found to explain how much such a contribution should be included as being part of the whole cuprate, or discarded for not coming from the superconducting planes.

From Figures 17 and 18 for the polycrystal and Figure 11 for oxygen we can estimate the quantity of oxygen needed to provide the observed rise in susceptibility with doping. For the BSCCO polycrystal in the temperature range between the onset of superconductivity and $200 \mathrm{~K}$ the increase in magnitude between the VHS and the doping level at $\mathrm{T}_{\mathrm{c}} \sim 60 \mathrm{~K}$ is approximately $0.2 \times 10^{-7}$ emu/Oe/g. Below $200 \mathrm{~K}$ the susceptibility of oxygen ranges from about 0.0625 to $0.175 \mathrm{~cm}^{3} \mathrm{~mol}^{-1}$ approaching superconducting temperatures. We can choose the value of $0.05 \mathrm{~cm}^{3} \mathrm{~mol}^{-1}$ of $250 \mathrm{~K}$ to increase confidence in the figure not being overestimated. The molar mass of oxygen will be taken as $16 \mathrm{~g}$.

First we must convert the oxygen susceptibility units:

$$
\begin{gathered}
\chi_{m}=\chi_{\text {volume }} \frac{M}{\rho} \rightarrow \chi_{\text {volume }}=\chi_{m} \frac{\rho}{M} \\
\chi_{g}=\frac{\chi_{\text {volume }}}{\rho}=\chi_{m} \frac{\rho}{M \rho}=\frac{\chi_{m}}{M}
\end{gathered}
$$

Here $\rho=$ density in $\mathrm{g} / \mathrm{cm}^{3}$ and $\mathrm{M}=$ molar mass, $\mathrm{g} / \mathrm{mol}$.

Therefore, as the Gaussian units are equivalent to cgs emu units:

$$
\chi_{g}(\text { oxygen })=\frac{0.05}{16}=0.003125=31250 \times 10^{-7}(\mathrm{emu} / \mathrm{Oe} / \mathrm{g})
$$


We would require $0.2 \times 10^{-7} \mathrm{emu} / \mathrm{Oe} / \mathrm{g}$ to get the effect we see, which would come from a quantity of $0.2 / 31250=6.4 \times 10^{-6} \mathrm{~g}$ of oxygen. Weighing of samples is done on a Mettler AE200 precision balance of readability to $0.110^{-3} \mathrm{~g}$, which is also the figure of uncertainty - variations in atmospheric pressure or placing a sample at different sites on the pan can vary readings by a few tenths of a milligram. Variation of the order of $10^{-5} \mathrm{~g}$ cannot be detected. This is the amount of completely free oxygen that would be required for the effect. It does not include magnetic contribution from oxygen in the bismuth oxide layer that has begun some level of chemical interaction with the cuprate, however weak these bonds may still be.

To detect a feature such as the VHS relies on comparisons, not on ability to accurately determine precise values. The very small doping shifts - or changes in oxygen levels - required to pass through the extremely narrow window in the VHS, while delicate to achieve empirically, do serve to accentuate the ratio of the change in magnitude to the changes in $T_{c}$ shift. The magnitude of the magnetic susceptibility through the VHS changes at a very much faster rate with respect to doping compared to other parts of the phase diagram. Thus, not subtracting a background does not detract much from detection and visibility of the VHS

\subsection{Single Crystal}

Measurements of $T_{c}$ in the single crystal were done under ZFC.

The single crystal $(\mathrm{Bi}, \mathrm{Pb})-2212$ failed to overdope, but nearer the end of the project was passed through a series of anneals 
comparable to the range of anneal conditions applied to the main polycrystal to see if it could still be used as a preliminary gauge as to the location of the VHS. The bulk $T_{c}$ barely moved but changing oxygen levels near the surfaces could possibly still provide information. During data collection, a control piece of polycrystal was included during these anneals as a comparative guide to the consequences of the conditions. As it is from the same solid state preparation as the main polycrystal it also served as a comparison for the alternative method of measuring $T_{c}$. The piece measured was small and flat in an attempt to mimic the shape of the single crystal and was half the mass of the main polycrystal sample.

The basic form of the $\mathrm{Bi}-2212$ magnetic response is evident in the data for the single crystal, as shown in Figure 25. This data does not show a smooth monotonic evolution with doping. In view of the dominant relative contribution of molecular oxygen to the susceptibility (see below) this erratic variation could simply arise from variations in adsorbed oxygen in a crystal which easily delaminates thus exposing large internal surfaces. 


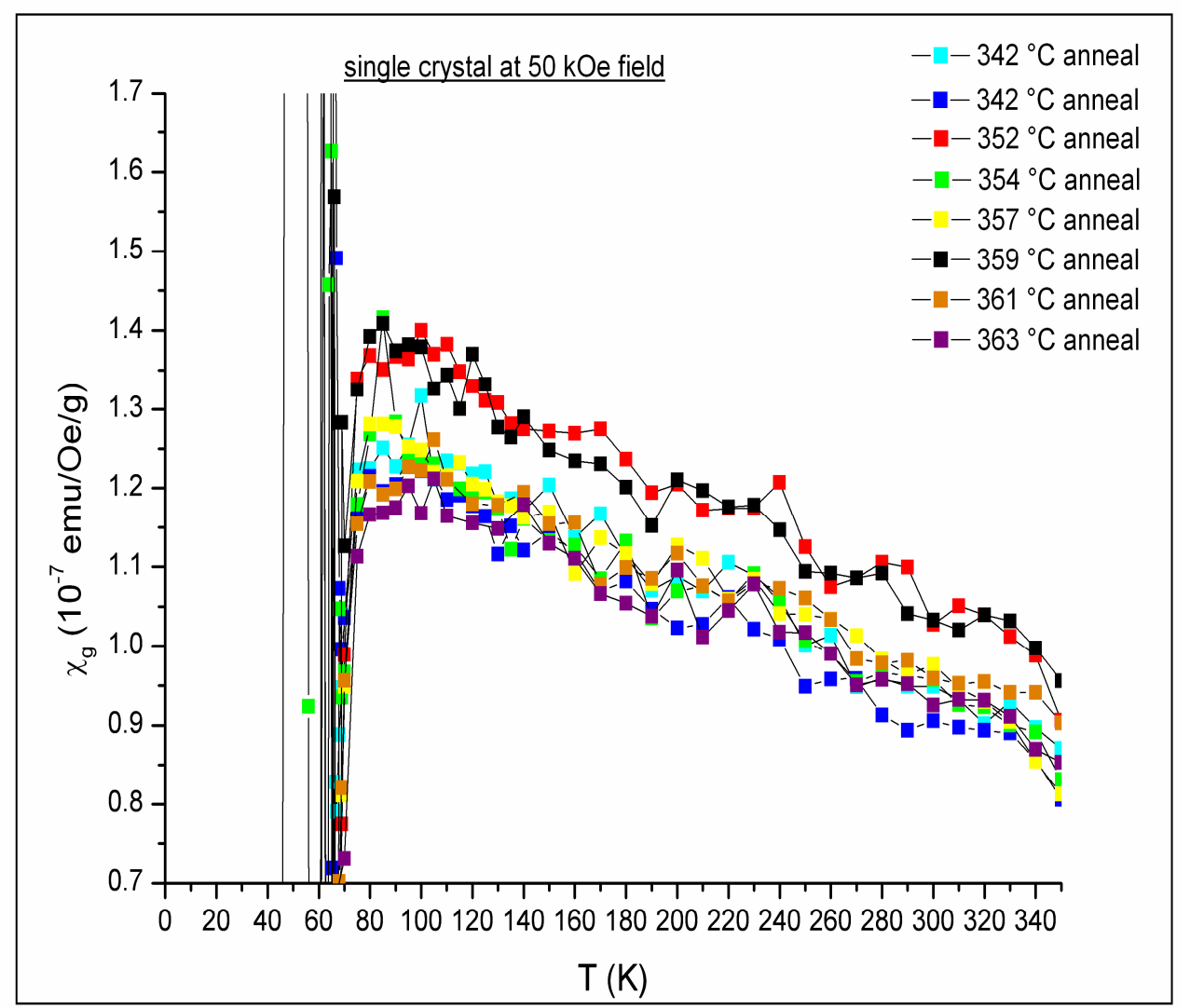

Figure 25. The temperature dependence of the normal -state susceptibility for the $(\mathrm{Bi}, \mathrm{Pb})-2212$ single crystal raw data at high field.

Figure 26 has detail of the low temperature region where it is particularly noticeable the action contributed by the changing oxygen levels. Note that the high-temperature data appears to coincide with each other. But when enlarged, as in Figure 25, the detail of the variation in normal-state susceptibility can be seen. 

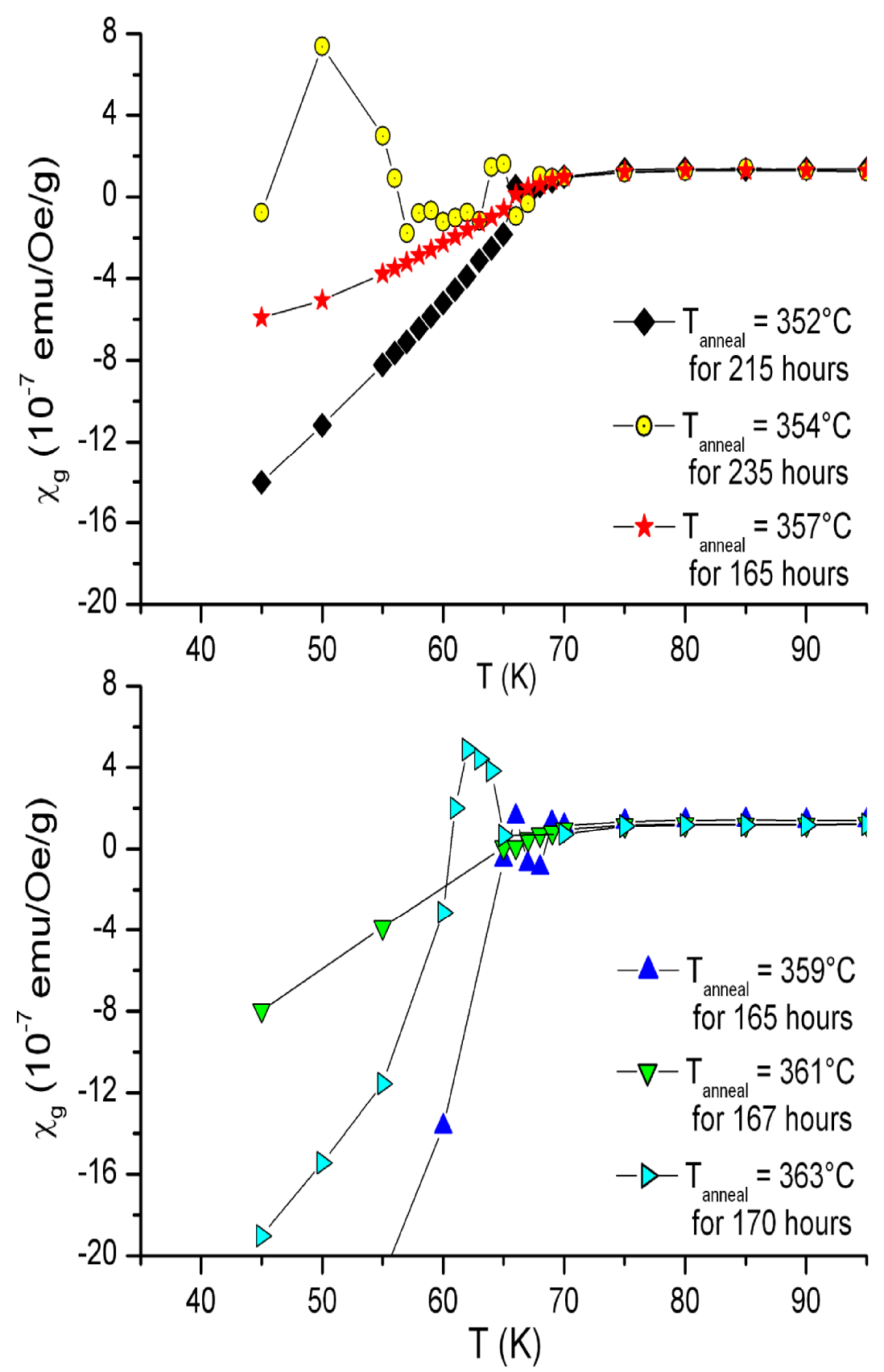

Figure 26. Low temperature T-dependence of the magnetic susceptibility for a progression of $(\mathrm{Bi}, \mathrm{Pb})-2212$ single crystal anneals. Samples are first aimed at extreme overdoping, then are annealed through a sequence of increasing temperatures to extract oxygen to move through decreasing doping steps. 
Of note in Figure 26 is the variability of the susceptibility below $T_{c}$ as the material is subjected to ongoing oxygen treatment. When the anneal temperature is changed from $352^{\circ} \mathrm{C}$ to $354^{\circ} \mathrm{C}$ the sample experiences more than 12 further days in the pure oxygen atmosphere. The magnitude of the susceptibility increases, the oxygen peak at around $50 \mathrm{~K}$ indicates that the apparent decrease in diamagnetism is due to the infiltration of molecular oxygen that has not yet completed chemical bonding with the cuprate. At the next anneal at $357^{\circ} \mathrm{C}$ the paramagnetism decreases again as there is less free oxygen and probably more oxygen chemically bonded into the structure. As time passes and the annealing steps progress, the bonding of atomic oxygen increases further and the paramagnetism of the oxygen begins to be overtaken by the diamagnetism of the superconductor. Finally, oxygen begins to penetrate far enough towards the middle of the crystal that movement of $T_{c}$ can be detected. The oxygen is continually entering the crystal and over time the dominance of the paramagnetism of the oxygen or the diamagnetism of the superconductor can compete until the bulk of the new chemical bonds are completed throughout the entire crystal and excess oxygen expelled.

The original anneal temperatures for the single crystal were between 300 and $315^{\circ} \mathrm{C}$, chosen because of their success in reaching a satisfactorily overdoped state in the polycrystals. Continued efforts with the single crystal at such temperatures failed to make headway in doping because of the low diffusion and the need for oxygen to diffuse on a scale of millimetres for the single crystal rather than micrometres for the polycrystal. The mistake was made of trying to 
anneal too far too soon, as works well for the polycrystal. Although the time constraints meant the single crystal was tested for the VHS before it was in a suitable overdoped state, it can be said that reaching the antibonding VHS in a single crystal appears to be possible. Aiming for an initial overdoping of a much warmer anneal temperature, such as 340 to $345^{\circ} \mathrm{C}$, would be beneficial. Here, the initial anneal conditions were aimed to reach doping beyond the "anomaly" at $T_{c} \approx 59 \mathrm{~K}$ to be able to test for this as well as the antibonding VHS, allowing also that these phenomena could be shifted a little compared to where they are found in the polycrystals. Start temperatures of $300-315^{\circ} \mathrm{C}$ were just too cool to make practical headway in doping a macroscopically sized crystal. The use of a HIP furnace to apply high pressure would also help enormously in achieving high doping levels in single crystal.

Plotting the susceptibility as a function of anneal conditions in lieu of critical temperature may demonstrate the placing of possibly both the VHS and, like the polycrystal, an anomaly at further overdoping. Due to the above noted circumstances the piece of single crystal did not dope sufficiently to demonstrate the monotonic rise in susceptibility. 


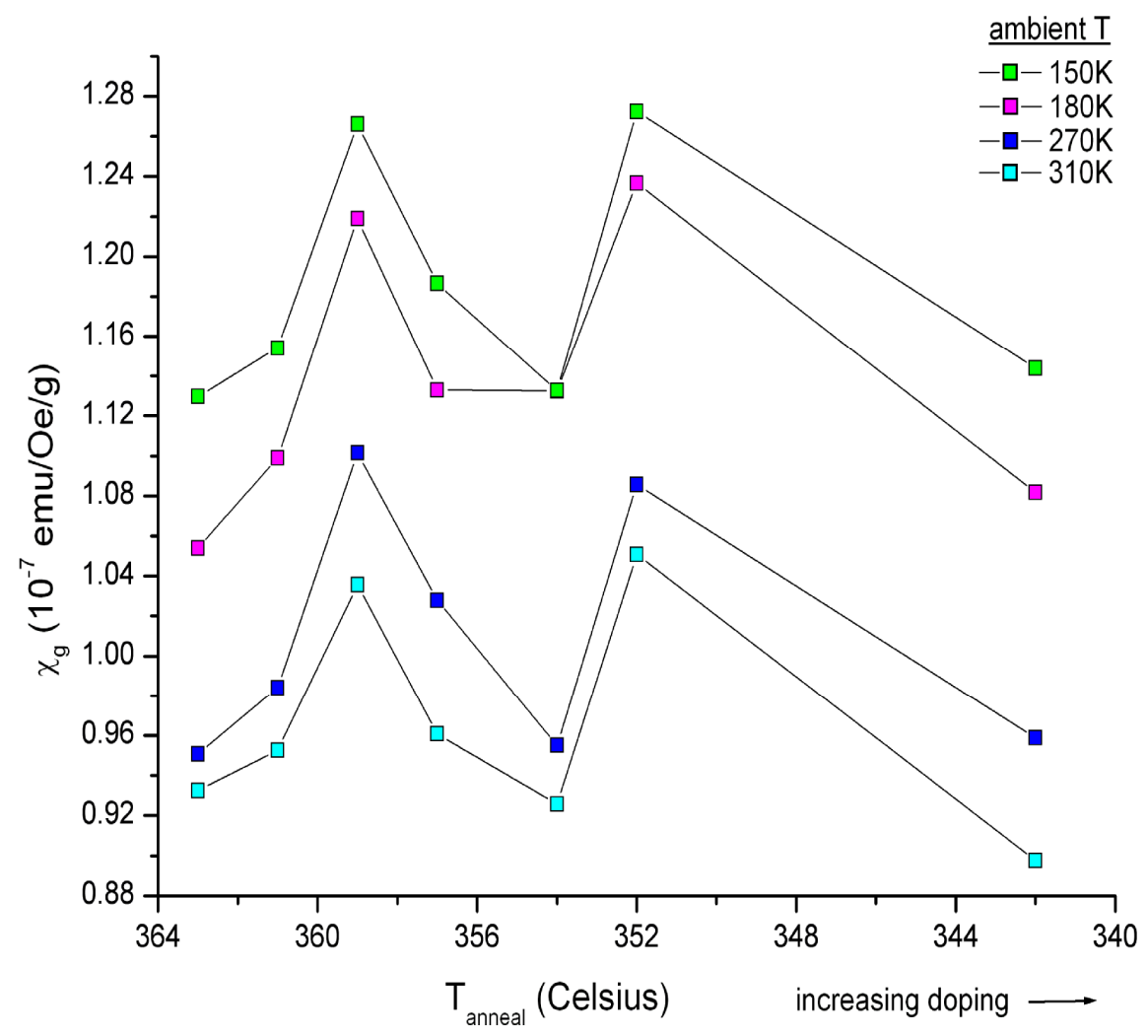

Figure 27. Single crystal susceptibility as a function of anneal conditions. The left hand peak is consistent with the VHS and the right hand possibly relates to the anomalous spike in the susceptibility seen in the polycrystals.

The erratic behaviour seen in Figure 27 may be due to variability in adsorbed molecular oxygen. Based on the calculations for the main polycrystal just $3.2 \times 10^{-6} \mathrm{~g}$ of oxygen, or $10^{-7}$ mole of $\mathrm{O}_{2}$, would be needed to account for the requisite changes of $10^{-8} \mathrm{emu} / \mathrm{Oe} / \mathrm{g}$ in the magnitude of the susceptibility. However, in spite of the ever changing oxygen levels the peaks only occur after weeks of annealing and only for specific anneals, after which the magnitude lowers again. The possibility remains that the VHS can be seen even through this level of oxygen activity. There also occurred a second spike during those weeks, which may correspond to the anomaly seen in the polycrystals. 
The spike that may correspond to the antibonding VHS occurs from anneal conditions appropriate to produce a VHS spike. The other spike is within the range of anneal conditions that have produced the anomaly in a polycrystal, but at exactly which anneal conditions will show the anomaly for a specific sample is possibly sample dependent. Without being able to measure the $T_{C}$ corresponding to this spike we can conclude little.

\subsection{Control Polycrystal}

As noted, after the single crystal was started on the series of anneals designed to pass through the VHS, a control polycrystalline sample of $(\mathrm{Bi}, \mathrm{Pb})-2212$ was included in with the same treatments. The aim was to obtain an idea of the $T_{c}$ produced by these conditions. The control polycrystal was from the same solid state preparation as the main polycrystal sample and served also as a reference for the FC polycrystal data. Figures 28-30 show control polycrystal data for comparison. The samples were put through a sequence of steadily increasing anneal temperatures. That is, there was no reannealing backwards and forwards through sites to gain an increased density of data points relating to a phenomenon. In the control polycrystal the VHS showed as a result of the anneal temperature of $361^{\circ} \mathrm{C}$ and the "anomalous spike" occurred from an anneal temperature of $357^{\circ} \mathrm{C}$. The single crystal "VHS" and "anomaly" spikes arose from anneal temperatures of $359^{\circ} \mathrm{C}$ and $352^{\circ} \mathrm{C}$ respectively. These shifts in temperatures compared to the polycrystal may indicate effects of different geometries. 


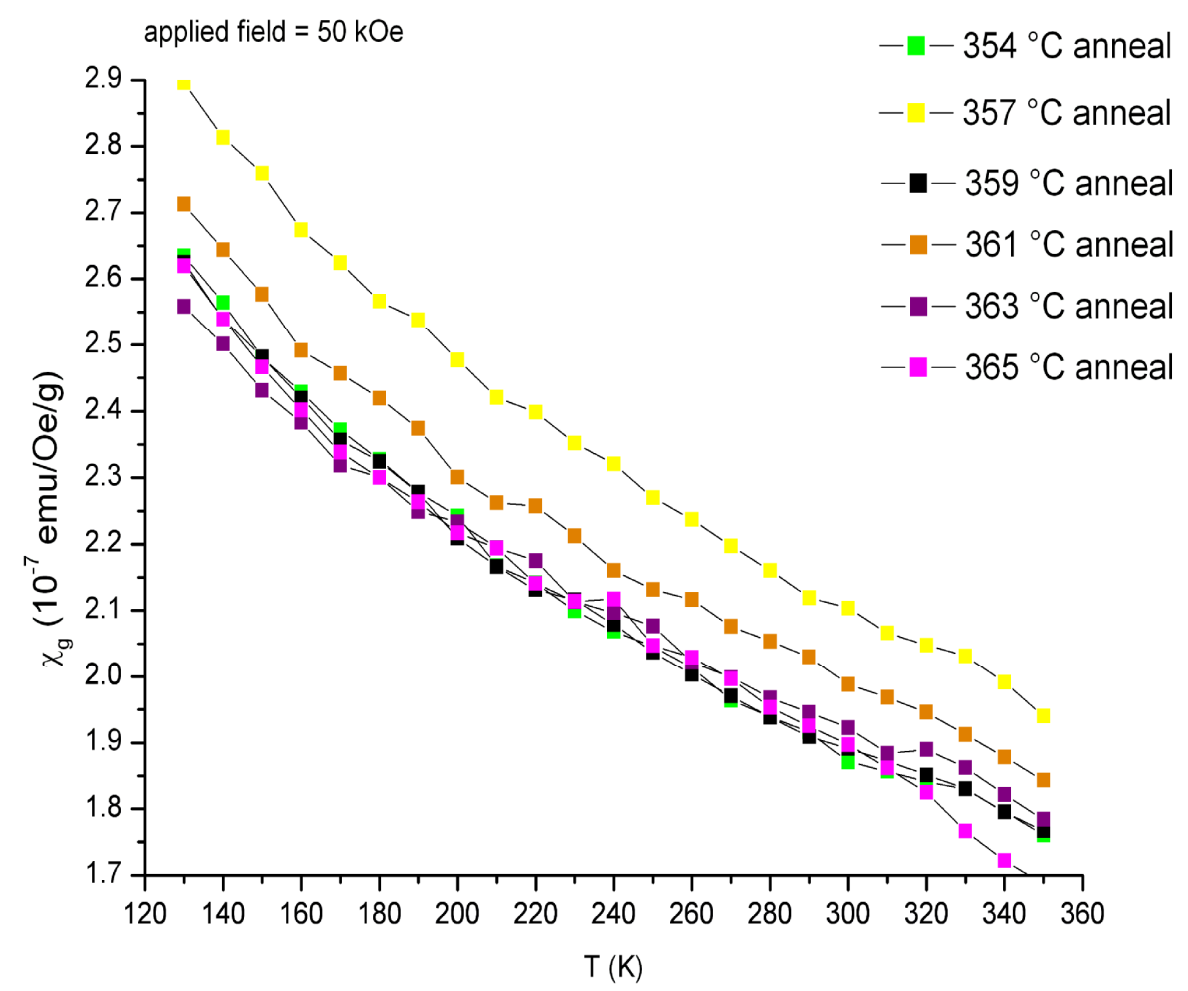

Figure 28. (Bi, $\mathrm{Pb})-2212$ Control polycrystal raw data.

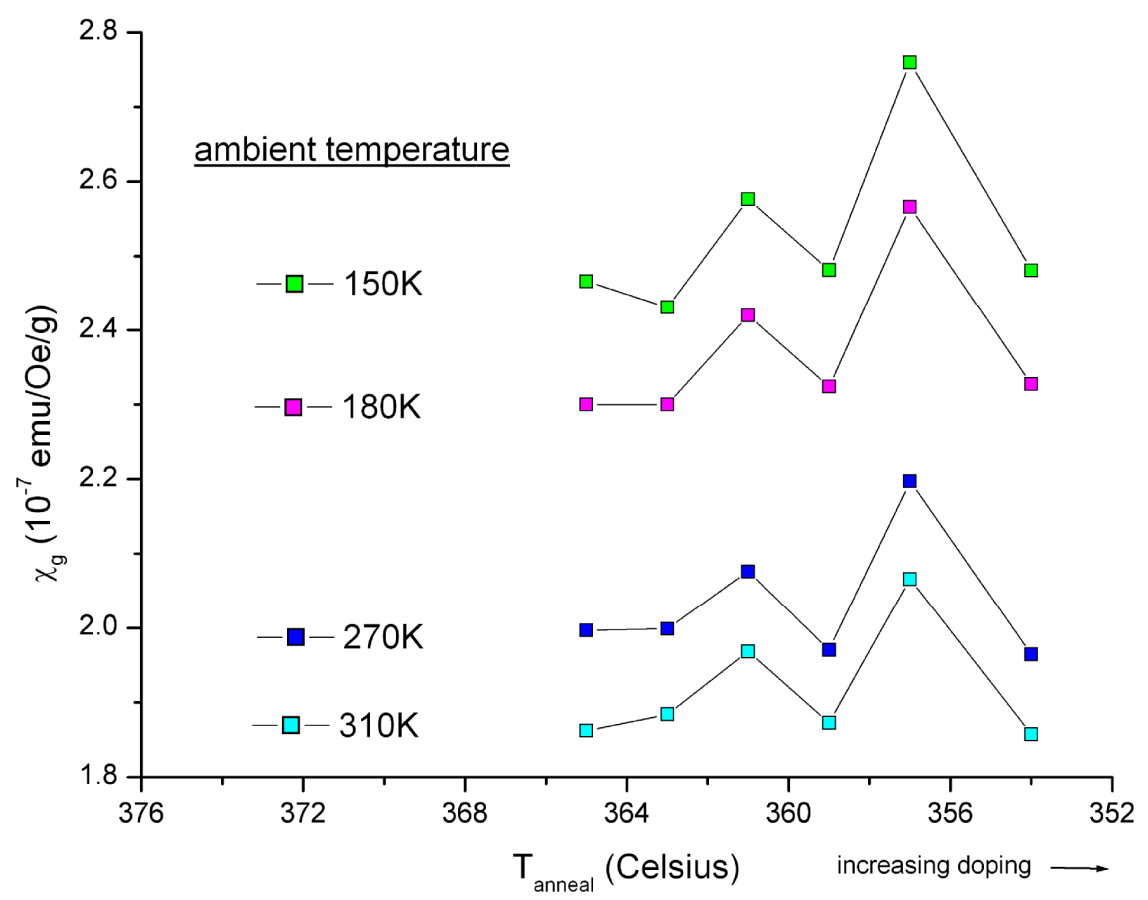

Figure 29. Control polycrystal susceptibility as a function of anneal conditions 


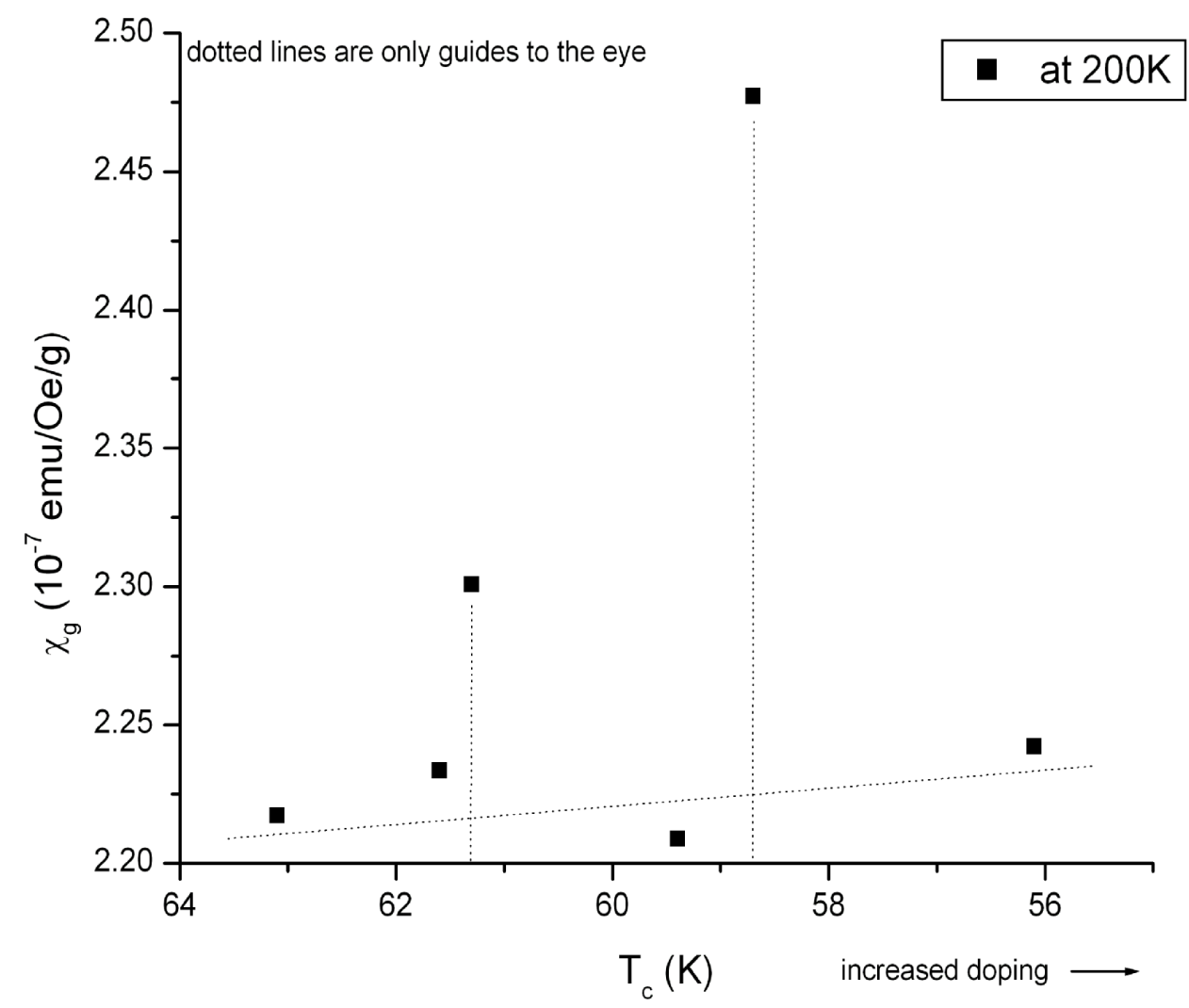

Figure 30. Control polycrystal VHS and anomaly of an example isotherm to show the comparison with the previous results of Figures 1 and 2 and the Main polycrystal of this experiment.

The control polycrystal confirms the location of the VHS peak at a $T_{c}$ a little greater than $61 \mathrm{~K}, \mathrm{ZFC}$, with a repeat of the unexpected spike in the magnetic susceptibility at a $T_{c}$ a little less than $59 \mathrm{~K}$. The monotonic increase with doping is also present.

Again, the VHS peak was visible in the susceptibility over only a very small range of doping. Precision control of anneal conditions could possibly be able to give quite high resolution of the peak. In practice this level of control is unlikely to be realised. The idealised situation would be to run the entire sequence of anneals in the same furnace with the same thermocouple and no external influence on the anneal 
temperature. This includes such considerations as no repairs to the equipment, no fluctuation in power supply and always the same level of usage of neighbouring furnaces. Furthermore, the anneal supply of oxygen flows through the oven with turbulence, which potentially subjects the sample to variations in pressure. Even for a polycrystal this can contribute to a sum total of such minor disturbances to the anneal history that is sufficient to be noticeable above the control needed to pass though the vHs. Small deviations on their own may be inconsequential to the result but a number of such contributions occurring simultaneously may add up to a sequence of temperature and pressure variations that result in the compound effectively being oscillated through heating and cooling, albeit by fractions of a degree, which noticeably disturbs the doping. Disturbance is also caused by the need to keep reannealing to pass several times through the VHS to get a significant number of data points.

As previously, the magnitude of the unexplained anomaly was higher than the VHS.

Ohkawa [60] commented, "Because of the large anisotropy of $\mathrm{CuO}_{2}$ planes, it is quite reasonable to argue that logarithmic van Hove singularities must be observed in various experiments. On the other hand, the absence of clear van Hove singularities in actual experimental data implies that logarithmic van Hove singularities in cuprate oxides are too weak to be observed." We find it not at all weak, though in magnetic susceptibility measurements in the $\mathrm{Bi}(\mathrm{Pb})-2212$ visibility is over an extraordinarily limited range of doping states. The VHS could be expected to be washed out as BSCCO is notoriously afflicted with disorder. Other cuprate HTS should offer clearer evidence still of the VHS in various normal-state properties. 
As the antibonding VHS is so well located by the use of Fermi liquid theory, we will consider it a reasonable guide also to the location of the bonding VHS, even though experiment has not yet been able to dope this far. For the Bi-2212, calculations using equation (1) show the bonding VHS occurs at a doping level of about $p=0.3$.

Through use of magnetic susceptibility we find the VHS is placed away from optimal doping, or away from where people were originally expecting to see it. Instead, we are finding the VHS rather nearer the vanishing of the superconductivity. Figure 31 illustrates the emerging trend.

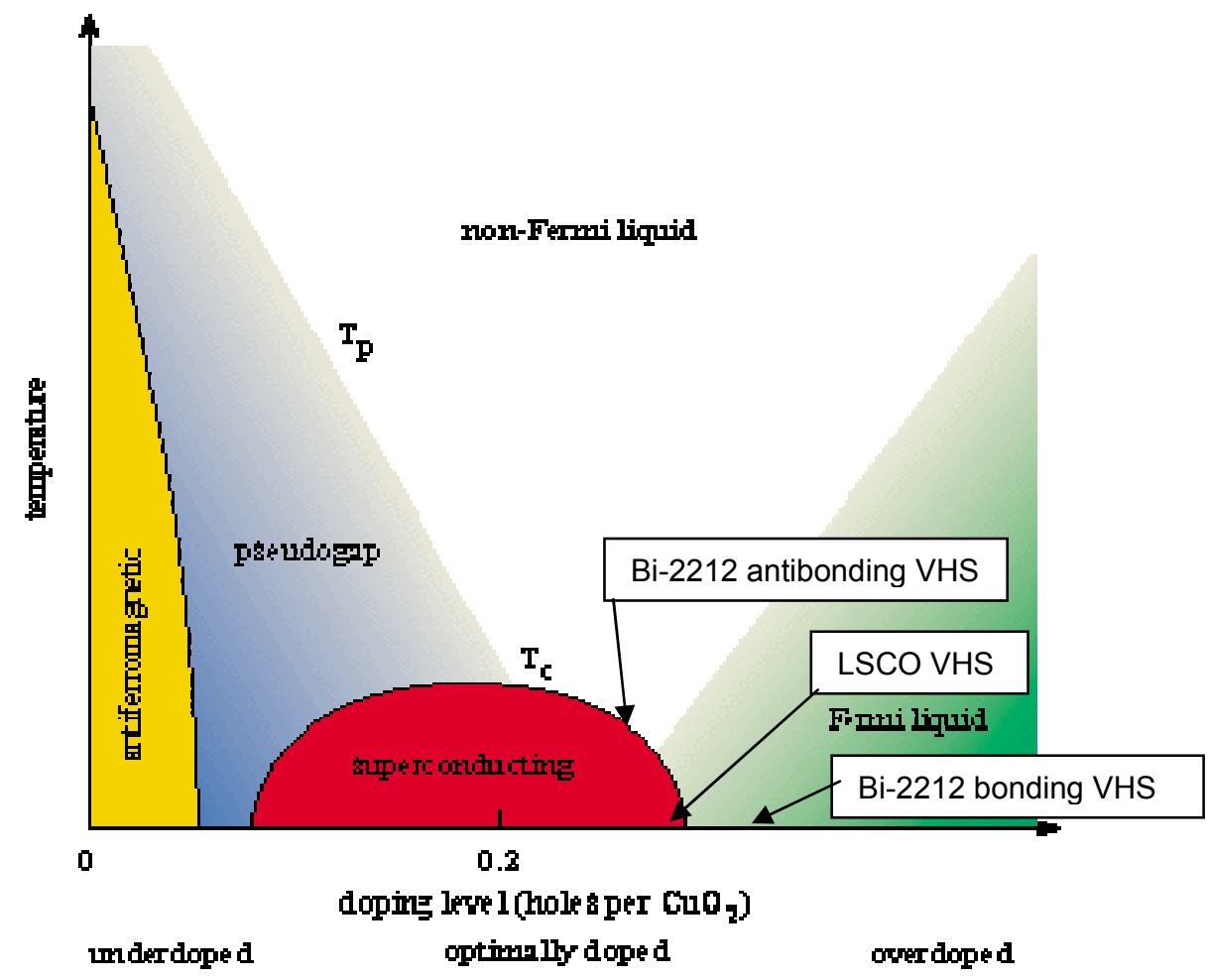

Figure 31. VHS locations. LSCO has one copper oxide layer. Bi-2212 has two copper oxide layers. Phase diagram reproduced from [16], copyright 2000 of IOP. VHS labels added 
(Note: If $T_{c}$ were to show increase with the increase in the DOS at the VHS, the peak would be shifted in relation to the foot. We do not see $T_{c} S$ out of sequence for either ZFC or FC measuring conditions such as for the examples shown in Figures 1,18 and 30. )

Further work is needed to confirm these results for other HTS materials, but so far, indications are that:

1. In single layer cuprates, the VHS will be located near the end of the superconducting dome, where $\mathrm{T}_{\mathrm{c}} \rightarrow 0 \mathrm{~K}$, rather than near the highest $T_{c}$ as suspected in the early 1990's.

2. In bilayer cuprates, the bonding VHS still retains a majoritycarrier Fermi surface, enabling the antibonding VHS to occur at a higher $T_{c}$, or closer to optimal doping. But the superconductivity will still collapse near the bonding VHS.

3. $T_{c}$ is not locally increased by the noticeable increase in the DOS provided by the VHS.

Such facts inspire a new look at the role of the VHS.

Other than the peak in the density of states, the very definite characteristic the VHS has is the change between hole-like and electron-like conductivity. 


\section{Research of Others}

\subsection{Normal state resistivity under the superconducting dome}

Boebinger et al. [64] tried to measure normal-state resistivity of the superconducting dome by using a $60 \mathrm{~T}$ pulsed magnetic field. Very high magnetic fields are required to suppress the superconductivity of the HTS materials. Until subjected to such treatment, the nature of the normal state under the superconducting dome remained a mystery. The results of Boebinger et al. for LSCO are shown in Figures 32,33 and 34 .

They find "..that all underdoped samples $(x<0.16)$ show low-temperature insulating behaviour,...". While this is true, we find inspection of the $x=0.15, x=0.17$ and $x=0.22$ data, for both $a, b$ and $c$-axis directions, shows remnants of superconductivity. In such a case, lack of insulating $\rho(\mathrm{T})$ behaviour can be expected. If Josephson coupling strengthens with doping it is conceivable a higher magnetic field becomes necessary to fully suppress regions of superconductivity. Indeed, Castellani et al. [65] also note that the data of the higher doping "is likely still affected by superconductivity effects". In other words the ground-state insulating behaviour persists beyond optimal doping. 


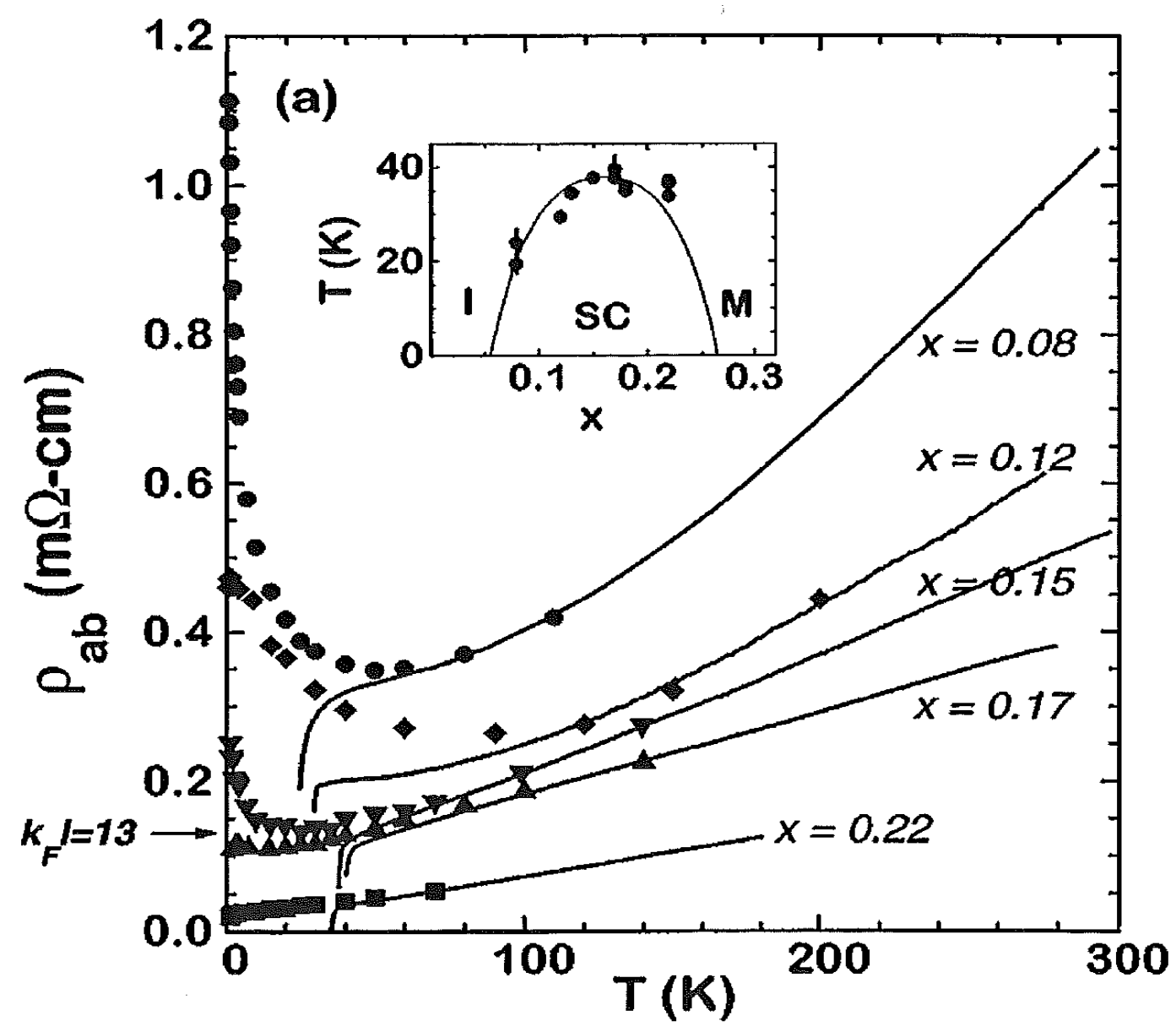

Figure 32. Resistivity data of Boebinger et al. [64] for the ab plane of LSCO. Reprinted (figure 1) with permission from, G. S. Boebinger, Yoichi Ando, A. Passner, T. Kimura, M. Okuya, J. Shimoyama, K. Kishio, K. Tamasaku, N. Ichikawa, and S. Uchida, Phys. Rev. Lett. 77, 5417. Copyright 1996 by the American Physical Society.. http://link.aps.org/doi/10.1103/PhysRevLett.77.5417 


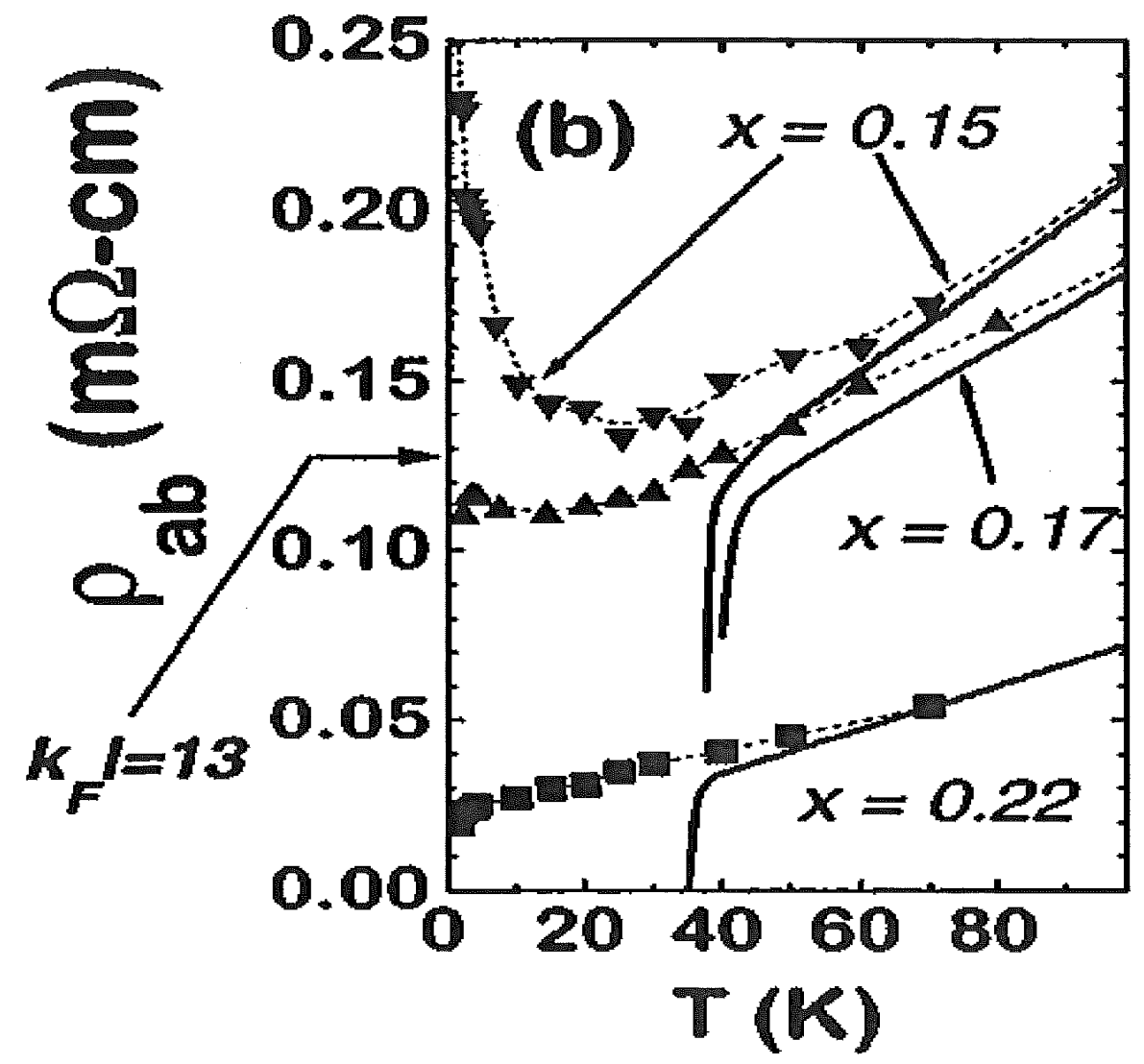

Figure 33. enlargement of the Boebinger ab-plane data for overdoped samples. At a doping of $p=x=0.17$ the resistivity at low $T$ appears affected by residual superconductivity which clearly in this case has not been fully suppressed. Reprinted (figure 1) with permission from, G. S. Boebinger, Yoichi Ando, A. Passner, T. Kimura, M. Okuya, J. Shimoyama, K. Kishio, K. Tamasaku, N. Ichikawa, and S. Uchida, Phys. Rev. Lett. 77, 5417. Copyright 1996 by the American Physical Society. http://link.aps.org/doi/10.1103/PhysRevLett.77.5417 


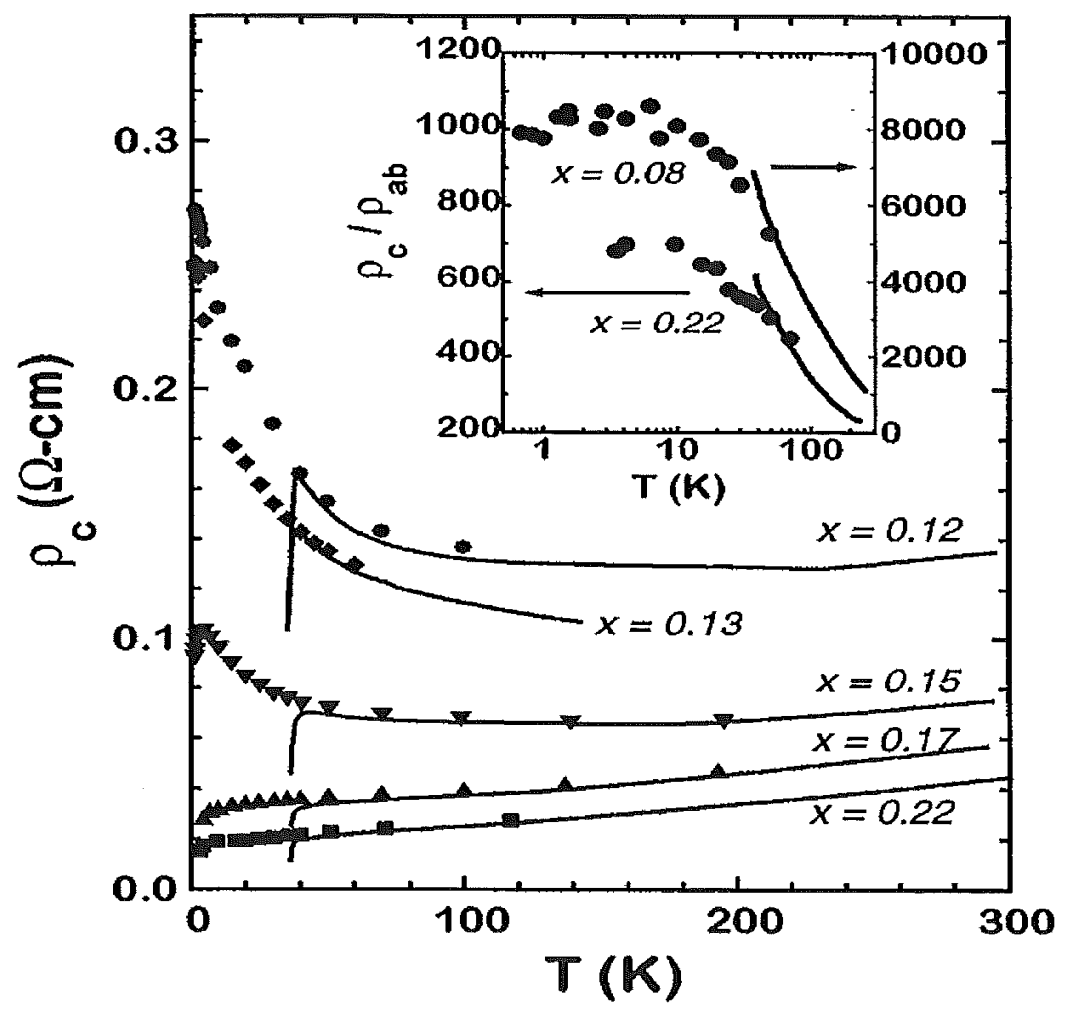

Figure 34. Resistivity data of Boebinger et al. for the c-axis direction of LSCO [64]. Superconductivity seems to be also affecting resistivity in this direction at the higher doping levels. Reprinted (figure 4) with permission from, G. S. Boebinger, Yoichi Ando, A. Passner, T. Kimura, M. Okuya, J. Shimoyama, K. Kishio, K. Tamasaku, N. Ichikawa, and S. Uchida, Phys. Rev. Lett. 77, 5417. Copyright 1996 by the American Physical Society.

http://link.aps.org/doi/10.1103/PhysRevLett.77.5417

The data of Boebinger et al. shows the material has both the temperature dependent MIT and the doping dependent change from insulator to metallic. Figure 35 shows resistivity as a function of temperature for a sequence of doped $\mathrm{Ca}_{1-x} \mathrm{Li}_{x} \mathrm{Pd}_{3} \mathrm{O}_{4}$ where the doping dependent MIT is the only MIT and is unaffected by MottHubbard physics. 
The normal state under the superconducting dome is difficult to even partially access for many of the cuprates due to the extremely high fields needed to repress the superconductivity. Or, in a Mott material, the temperature dependent MIT could be evaluated in part by measurements of bond length as a function of temperature at different doping levels. A Mott transition from metallic to insulator will be accompanied by increasing bond length.

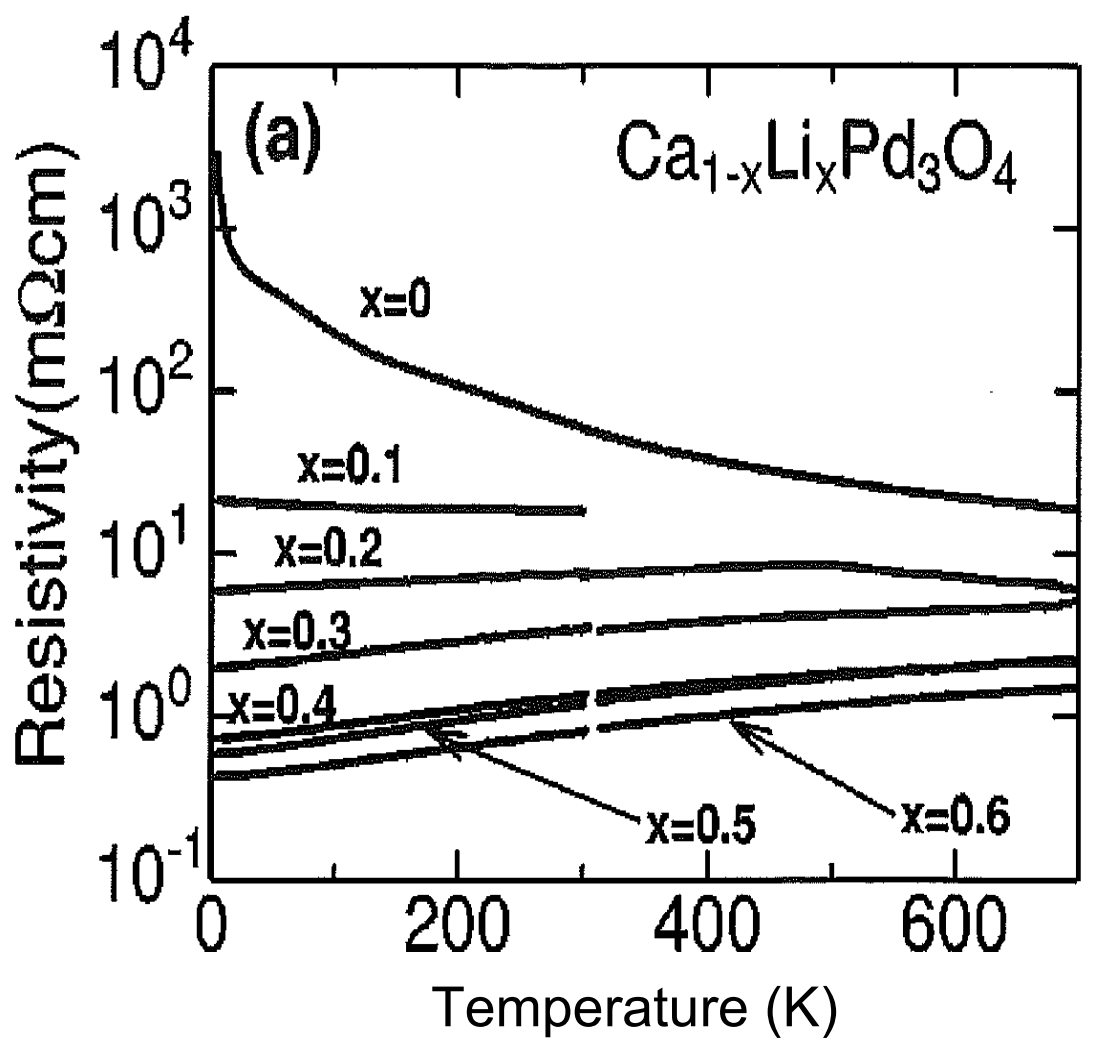

Figure 35 Ichikawa and Terasaki find the doping dependent MIT of $\mathrm{Ca}_{1-x} \mathrm{Li}_{x} \mathrm{Pd}_{3} \mathrm{O}_{4}$ between doping levels of $x=0$ and $x=0.1$ [66]. Reprinted (figure 3a) with permission from, S. Ichikawa and I. Terasaki, Phys. Rev. B 68, 233101. Copyright 2003 by the American Physical Society. http://link.aps.org/doi/10.1103/PhysRevB.68.233101 
Meanwhile, the normal state resistivity $(\rho)$ has also been measured under the dome for an electron doped cuprate, $\mathrm{Pr}_{2-x} \mathrm{Ce}_{x} \mathrm{CuO}_{4}$, [67], where a lower field is needed to suppress the superconductivity. For this cuprate too, $d \rho / d T$ of the ab plane is found to be negative (the insulator-like characteristic) also at less than optimal doping, which they find to be near a MIT. The behaviour of the c-axis resistivity with doping is not mentioned.

It appears the resistivity changes steadily as the material evolves from insulator to metallic, and these insulator characteristics persist even into the superconducting dome. This is seen in most, possibly all HTS.

\subsection{Cuprate Bond-length Data}

The Mott MIT can be physically observed in terms of a critical bond length. Fijalkowski and Grochala [68] have collected a multitude of experimental data correlating bond length with superconductivity in the cuprates. Their data is presented graphically in Figure 36. 


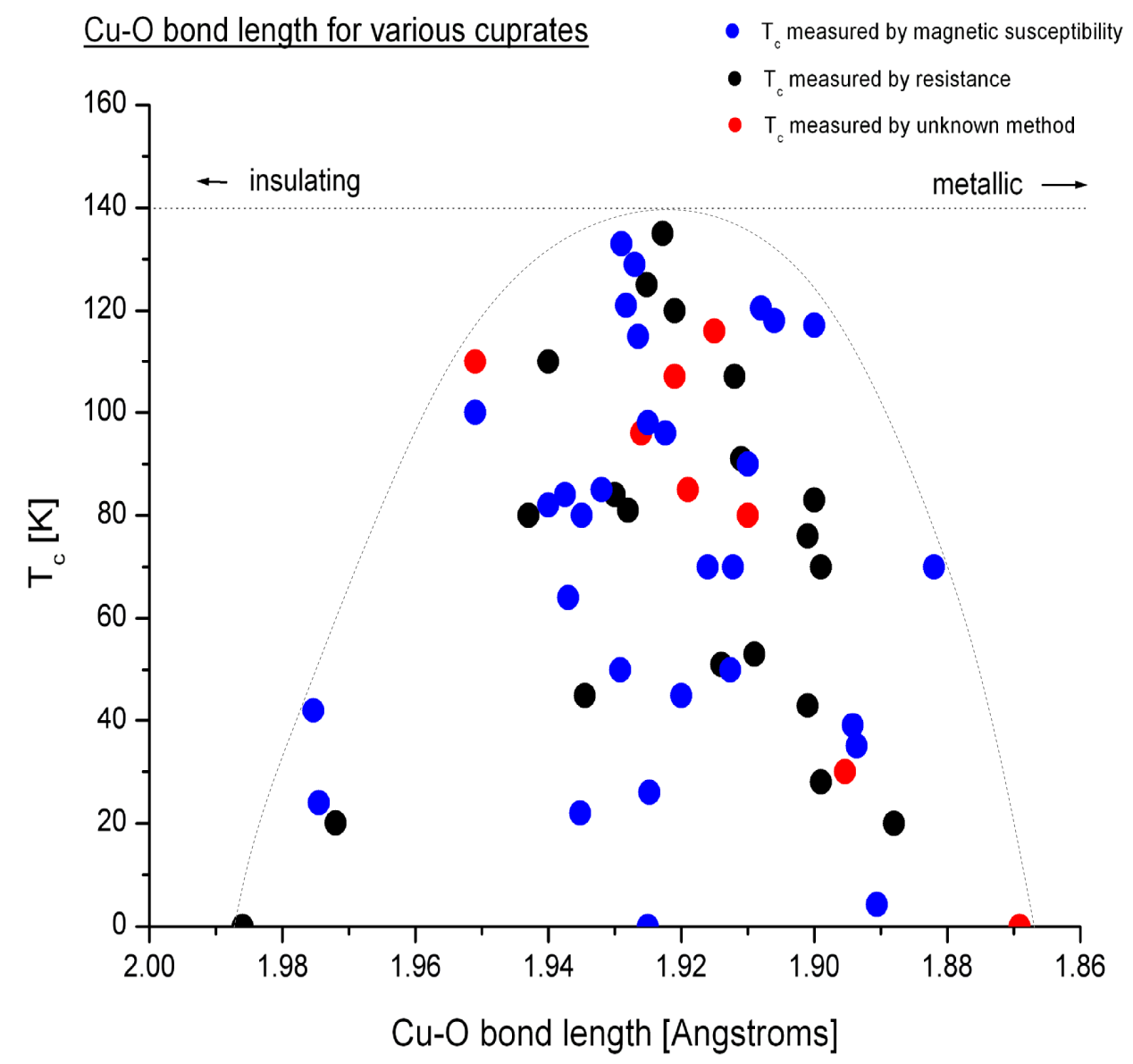

Figure 36. Relation of $\mathrm{T}_{\mathrm{C}}$ to $\mathrm{Cu}-\mathrm{O}$ bond length for various hole-doped and electrondoped cuprates. Data from [68]; Karol Fijalkowski and Wojciech Grochala, The 'magic' electronic state of high-Tc cuprate superconductors, Dalton Tansactions 2008, DOI: $10.1039 / \mathrm{b} 802879 \mathrm{f}$ - Reproduced by permission of The Royal Society of Chemistry Below $140 \mathrm{~K}$ the antiferromagnetism of cupric oxide is independent of temperature. The dashed line marking out a dome shape is intended only as a guide to the eye.

They find an optimal bond-length of $1.92 \AA$, which they dub a "magic" bond-length. In Figure 37 is shown how $T_{c}$ varies with $\mathrm{Cu}-\mathrm{O}$ bond length for six of the cuprate families. 

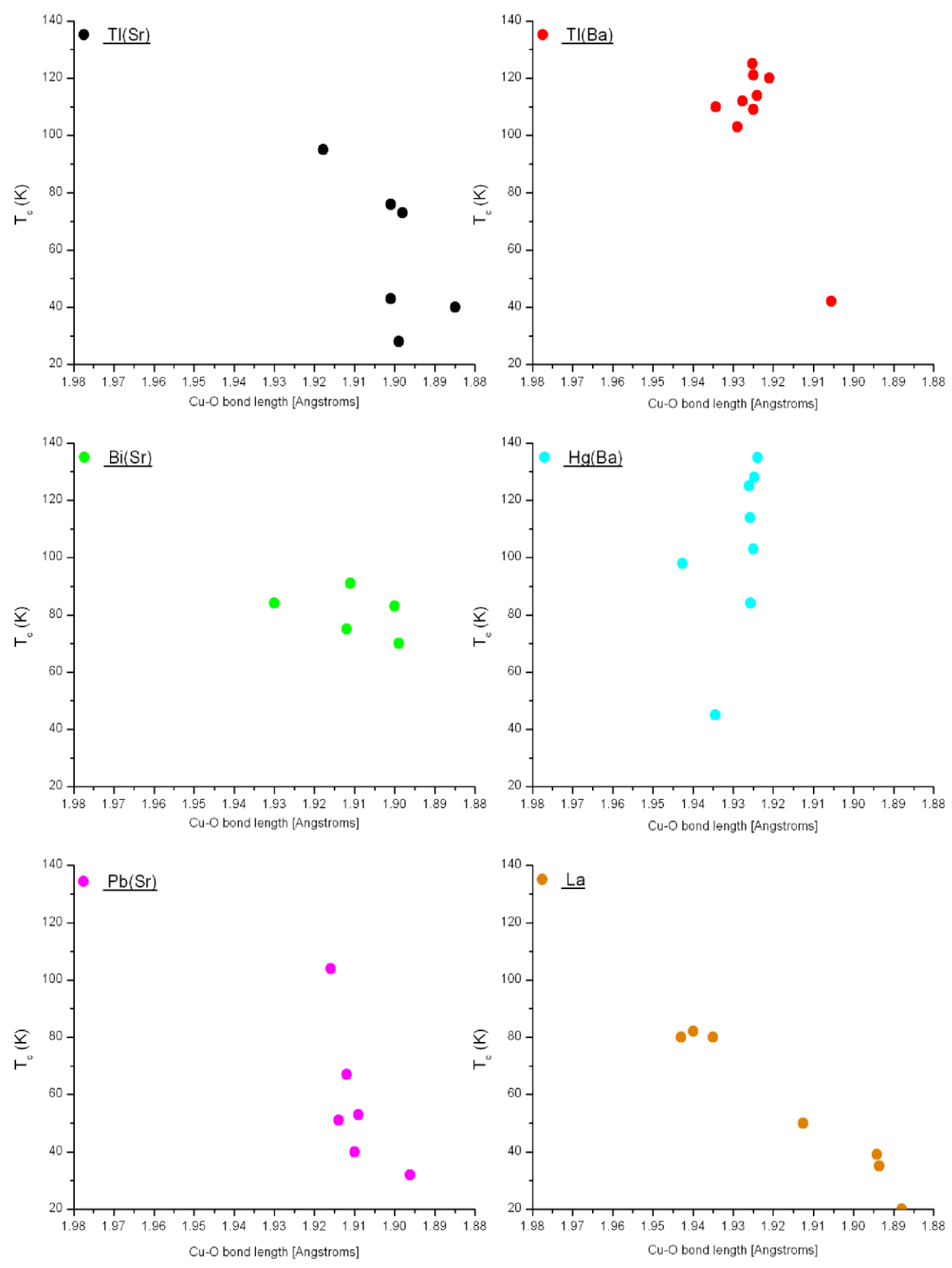

Figure 37. $T_{c}$ vs bond length for six cuprate families. Use of data from [68]; Karol Fijalkowski and Wojciech Grochala, The 'magic' electronic state of high-Tc cuprate superconductors, Dalton Tansactions 2008, DOI: 10.1039/b802879f Reproduced by permission of The Royal Society of Chemistry. The members of the La family with the bond length near $1.94 \AA$ incorporate both $\mathrm{Ba}$ and $\mathrm{Ca}$ in their stoichiometry. 
These materials show a generalised trend of either decrease in $T_{c}$ as bond length decreases (becomes more metallic) from 1.92Á, or increase in $\mathrm{T}_{\mathrm{c}}$ as bond length approaches $1.92 \hat{A}$ from the insulating side. Those that approach this bond length from the insulating side have a tendency to achieving higher $T_{c} S$ than those that approach Fijalkowski and Grochala's "magic" bond length from the metallic side. Bearing in mind Mott's critical bond length, the superconductivity of the material appears to be correlated to the resistivity of the normal state, which here shows as a generalised trend over a wide range of the known HTS.

\subsection{Superconductivity as a function of normal state resistivity}

As noted earlier and shown in Figure 38, the phase diagram of the cuprates could be instructively presented as a function of temperature $(T)$ and resistivity $(\rho)$, instead of temperature $(T)$ and doping ( $p)$. Alternatively, the view could be the phase diagram is a function of temperature $(T)$ and conductivity $(\sigma)$. Resistivity is, of course, a function of temperature as well as of doping, but we can focus our attention on how the resistivity changes with doping for a fixed temperature and repeat this activity for a variety of temperatures. The important matter is awareness of how the superconductivity changes as normal state conductivity varies with doping.

The closure of the pseudogap (the point where the pseudogap $T^{*}$ line extends to $\mathrm{OK}$ in Figure 38) in the lightly overdoped regime is considered by many to possibly mark the existence of a quantum critical point. At $x(=p)=0.18$ or 0.19 is where the pseudogap closes 
in many HTS [69]. Using LSCO as a model, Uchida [14] puts this critical point at doping of around $x=0.18$ and remarks, "Nothing is special near $x=0.18$, but a different type of anomaly starts to develop when $x$ exceeds $\sim 0.18$."

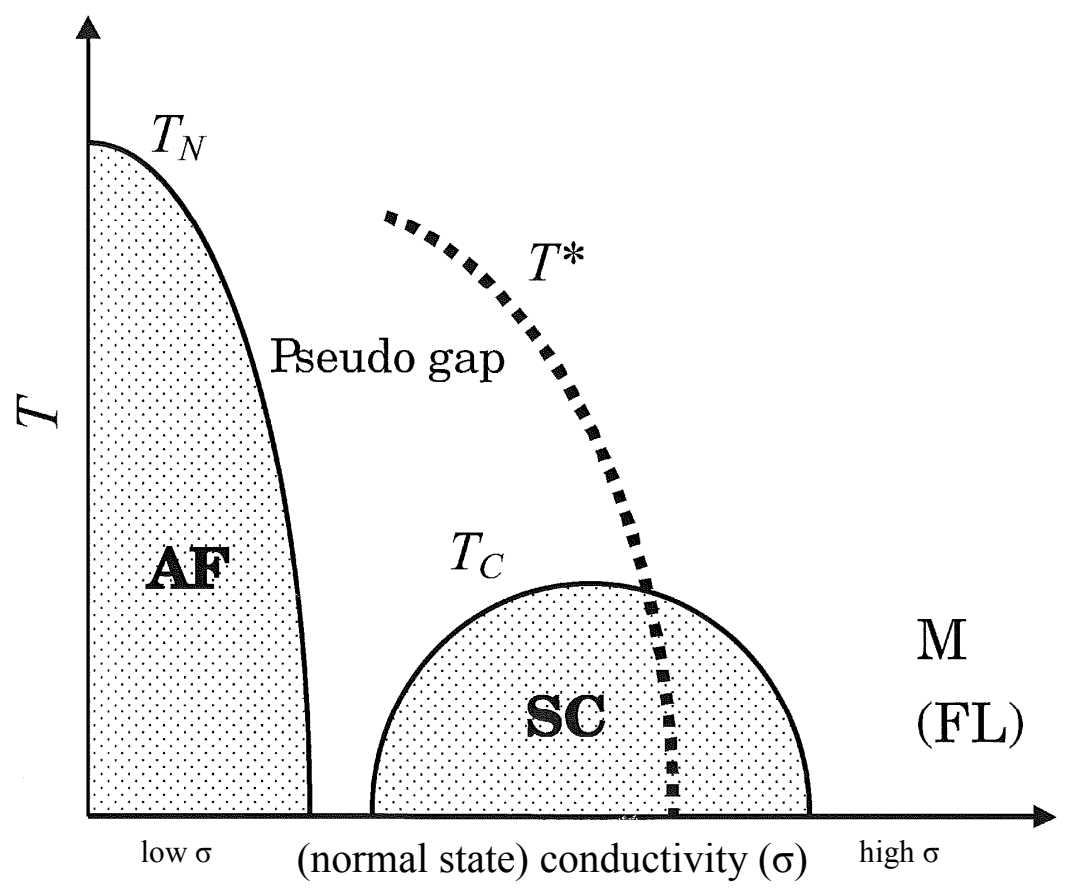

Figure 38. Cuprates phase diagram adapted from S. Uchida [14] showing trends with increasing conductivity. ${ }^{1}$

${ }^{1}$ Reprinted [with adaptation to axes] from Solid State Communications 126. S Uchida, Critical points in the cuprate phase diagram, pp 57-61. Copyright (2003) with permission from Elsevier. 
Granular aluminium can have its $T_{c}$ measured as a function of resistivity, which traces out a dome [34]. In their study of the MIT in granular aluminium Dynes and Garno [34] find maximum $T_{c}$ in the granular aluminium at resistivity where $\mathrm{k}_{\mathrm{F}} \lambda=1$, where $\lambda$ is the mean free path, a parameter important with regard to properties of metallic superconductors e.g. [33], and note it is near here Mott predicts an abrupt MIT. Their comment is, "Although it is clear that nothing precipitous happens here, it is at this point that there are changes in the material which ultimately result in the metal-insulator transition at a resistivity three to four orders of magnitude higher." They do not show the superconductivity data to this point but state that the MIT is where the superconductivity vanishes.

Granular superconductors consist of grains of metal - metallic BCS superconductors - covered with an insulating layer. An ideal granular superconductor has grains of all the same size. The superconductivity is controlled by the grain size and the thickness of the insulating layer that covers the grains.

Deutscher [33] considers comparison of granular superconductors with the HTS superconductivity to be "instructive". It is certainly interesting to consider the superconductivity of various materials as a function of resistivity. He also comments [p 64, 33], "It is in general difficult, and in fact practically not possible, to start from an arbitrary metal and to bring it up to a M/l transition of that kind by the use of some control parameter."

While going as far as a MIT within a single element may be not possible, at least for now, we may still be able to acquire empirical measurement of $T_{c}$ versus resistivity for elements that superconduct. Buzea and Robbie [5] compile data for the superconductivity of 
many elements as a function of pressure, and record a similarity between pressure and doping, at least in its effect on the development of superconductivity in ferromagnetic and antiferromagnetic materials. Even with the various methods newly applied to the elements to induce superconductivity, the very good metallic conductors copper, silver and gold remain unable to superconduct.
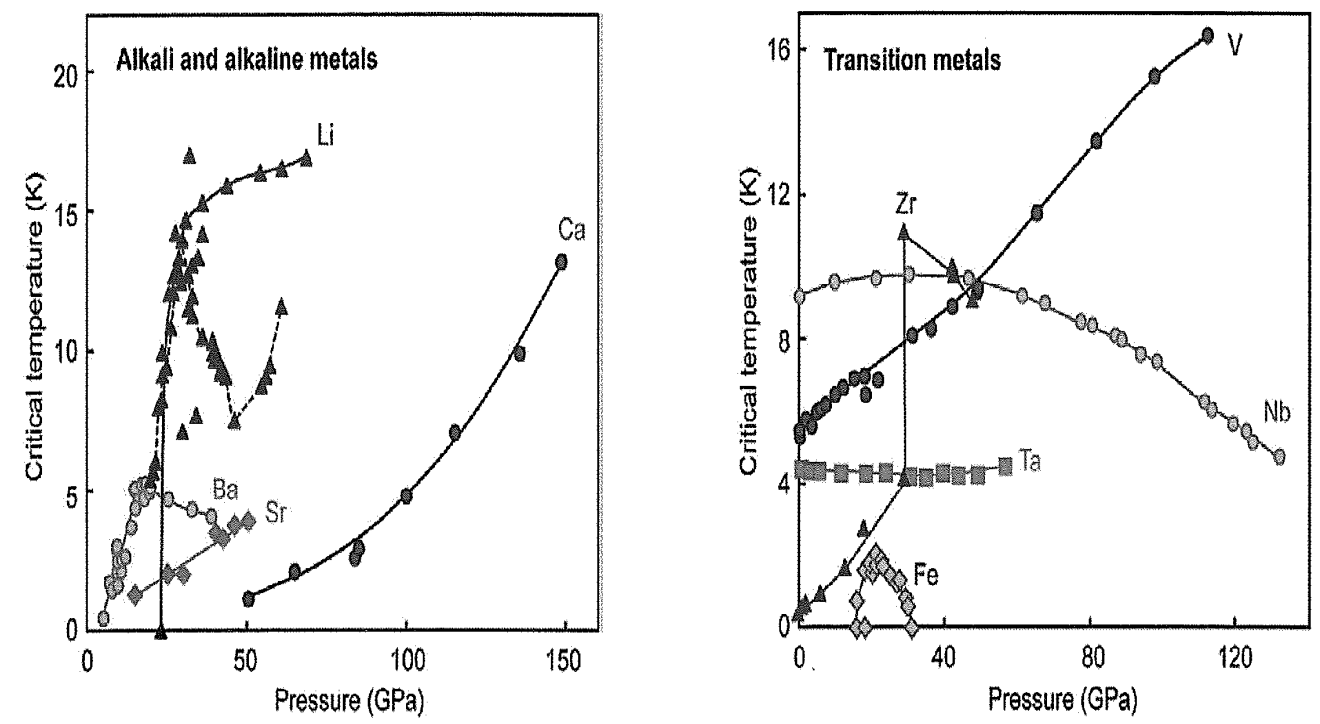

Figure 39. Examples of $T_{c}$ versus pressure curves for elements. Reproduced from [5] with permission. Copyright 2005 IOP Publishing.

The original metallic superconductivity did better in the "bad" metals. It would be interesting to return to the elements with the goal of concurrently measuring resistance and/or bond length as $T_{c}$ changes with increasing pressure.

The superconducting $T_{c}$ relationship with normal state electrical conductivity is rather spread, but the effects of pressure on 
superconductivity in the elements are concentrated on those of lower normal state conductivity (Figure 40).
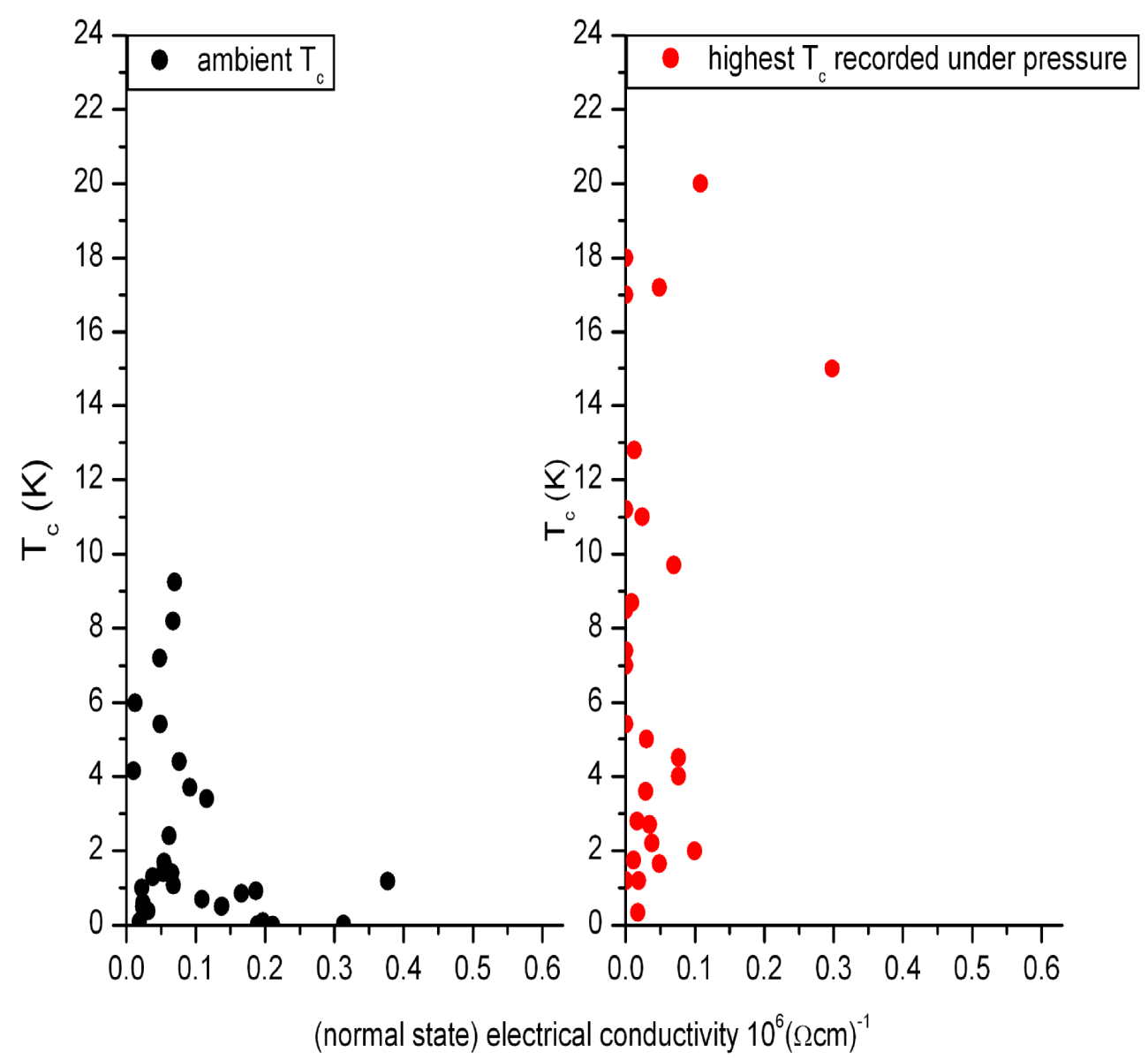

Figure 40. Superconductivity vs normal state conductivity for the elements under both ambient conditions and applied pressure. The stray element with electrical conductivity of $0.3 \times 10^{6}(\Omega \mathrm{cm})^{-1}$ that still can reach a $T_{c}$ of $15 \mathrm{~K}$ is calcium, and the pressure needed for this is $150 \mathrm{GPa}$. Permission granted for use of data: the superconductivity $T_{c} s$ from [5], normal state conductivities from [70].

It has been noticed the success of pressure in producing high(er) $T_{c} S$, or simply to induce superconductivity, and the point of view has developed that pressure may have equivalence to doping. Since the HTS materials of interest grow out of Mott insulating transition-metal 
compounds we can see what information arises out of changing our perspective to one that doping may have equivalence to pressure and turning to the extensive research available from the efforts of the earth sciences.

Transition metals and transition metal compounds comprise a significant part of the interior of the planet. Although the copper compounds have not been their first choice for study, geophysics has been enthusiastically submitting materials of transition metals to high pressure studies ever since it was first possible to control such parameters under laboratory conditions. Iron compounds in particular are very well examined and in spite of the differences between the ferromagnetic iron and the diamagnetic copper, a look at the knowledge gained by the earth sciences about transition metal compounds, Mott insulators and the Mott transition may help to resolve some of the HTS questions. Studies include the antiferromagnetic hematite, $\mathrm{Fe}_{2} \mathrm{O}_{3}$ and the even valence $\mathrm{FeO}$. Since the discovery of the iron based HTS [71], one of the proposals that has risen in HTS is that even valence of the transition metal within the compound has importance to the development of the superconductivity. Geophysics high pressure studies find that materials of either odd or even valence of the transition metal can pass differently through the Mott MIT. 


\subsection{Doping compared to Pressure}

\subsubsection{Magnetic breakdown as a function of pressure}

Pasternak and Taylor [72] were particularly interested in studying the high pressure transition as it occurs in Mott insulators. They conclude:

“...it is possible to obtain clear details of the HP [HP = High Pressure] state of magnetism in Mott insulators. It was shown that those materials that do not undergo a spontaneous Mott transition which results in a magnetic collapse, e.g., $\mathrm{Nil}_{2}, \mathrm{Fe}_{2} \mathrm{O}_{3}$, will eventually undergo a $\mathrm{HS} \rightarrow$ LS [HS = High Spin, LS = Low Spin] transition. In the case of even-valence configurations the spin-crossover will lead to diamagnetism and in the case of odd-valence configuration, to the gradual detrioration of the direct exchange and superexchange interactions and to the ultimate closure of the Mott-Hubbard gap." (itallics theirs)

They attribute the appearance of diamagnetism in systems with an even number of d-electrons to a transition from high spin to low spin as the decrease in interatomic distances causes crystal field splitting. For example, Hund's rule breaks down in FeO. The high pressure leads to higher energy densities, which in the transition metal compounds leads to the splitting of the d-orbital energy levels. When the energy levels are separated by a large gap - a big split - it is difficult for electrons to reach the higher energy and it is energetically more favourable for the electrons to pair spin up/spin down to fill the orbitals of the lower levels. This pairing formation is termed "low spin". When the energy splits by a small amount so the levels are separated by a small energy gap it is much easier for an electron to attain the higher energy level, and it is energetically favourable for electrons to jump this gap to place one electron in each orbital with the spins all in one direction, before filling orbitals with paired spin. This is the "high spin" formation. 
Pasternak and Taylor consider many transition metal compounds including (alpha-) hematite, $\mathrm{Fe}_{2} \mathrm{O}_{3}$ which "with its large optical gap can be regarded as archetypical of a Mott insulator.", and the general group $\mathrm{RFeO}_{3}$, where $\mathrm{R}$ is a rare earth element. These compounds overall are "well known antiferromagnetic insulators with very large optical gaps." It may be observed that with some of the oxygen replaced by alternatives such as arsenic and fluorine, the valence of the iron the transition metal - is altered and superconductivity may be attained (for example La $\left(\mathrm{O}_{1-\mathrm{x}} \mathrm{F}_{\mathrm{x}}\right) \mathrm{FeAs}$, -or LaFe $\left(\mathrm{O}_{1-\mathrm{x}} \mathrm{F}_{\mathrm{x}}\right) \mathrm{As}$ [73]instead of $\mathrm{LaFeO}_{3}$ )

Takele and Hearne [74], motivated by the conditions in the interior of the earth, concentrate a study on the changes in FeS under high pressure. FeS is stoichiometric. They do note that transition metal sulphides can be compared with transition metal oxides. Both oxygen and sulphur are chalcogens. Incidentally the valence of iron in this compound is $2^{+}$. Briefly, a magnetic signature appears at $\sim 3$ $\mathrm{GPa}$ (with a dramatic jump in conductivity) at room temperature and disappears at $\sim 7 \mathrm{GPa}$. Their ability to monitor the changes down to a temperature of $5 \mathrm{~K}$ lead them to describe the nonmagnetic state at $\mathrm{P}>7 \mathrm{GPa}$ as being diamagnetic. For this compound resistivity measurements showed that throughout the attainable pressure range $d R / d T$ remained negative.

\subsubsection{Conductivity as a function of magnetic breakdown}

Patermarakis et al. [75] study the magnetic transition of pure hematite without pressure as a variable. In $\alpha-\mathrm{Fe}_{2} \mathrm{O}_{3}$ the Néel temperature occurs at $956 \mathrm{~K}$, with what is known as the Morin transition at $\sim 265 \mathrm{~K}$ where the magnetic ordering changes from 
perpendicular to parallel to the c-axis. Magnetic susceptibility is approximately constant at $\mathrm{T}>\sim 275 \mathrm{~K}$ with a dramatic drop below $265 \mathrm{~K}$, then levels off to constant again with much decreased moment at $\mathrm{T}<\sim 225 \mathrm{~K}$. At the low temperature constant-moment state the material is in the antiferromagnetic phase. In the constantmoment phase at higher temperatures above $275 \mathrm{~K}$ the net magnetic phase is weakly ferromagnetic, due to canting of the perpendicularly aligned spin. The change from the ferromagnetic to the antiferromagnetic region is called a spin-flip transition and they found this magnetic transition to take place over a significant temperature range, extending from $\sim 230 \mathrm{~K}$ to $\sim 330 \mathrm{~K}$ (exact endpoints dependent on parameters and definition).

This type of behaviour is observed in the magnetic superconductor $\mathrm{RuSr}_{2} \mathrm{GdCu}_{2} \mathrm{O}_{8}[76]$

Patermarakis et al. were particularly interested to see how electrical conductivity develops with the change in temperature and to correlate it with the magnetic transition. They found a wide peak in the conductivity (or equivalently, a wide inverse peak in resistivity) that spikes around the Morin transition, the temperature of conversion between weak ferromagnetism and (strong) antiferromagnetism. This occurs without pressure or doping. - The conductivity jumps at the magnetic transition.

They point out that overall the magnetic and conductivity transitions occur concurrently. 


\subsubsection{Conductivity as a function of Pressure}

Pasternak and Taylor [72] measured the resistance of hematite as a function of pressure. From $\mathrm{P}=42 \mathrm{GPa}$ to $\mathrm{P}=59 \mathrm{GPa}$ the resistivity dropped - or the conductivity increased - by several orders of magnitude. At $\mathrm{P}<42 \mathrm{GPa}$ the resistance was insulator-like and at $\mathrm{P}>59 \mathrm{GPa}$ was metallic, as confirmed by $\mathrm{dR} / \mathrm{dT}$ curves. For the intermediate pressures the material showed a mixture of phases. Incidentally, their Mössbauer spectra as a function of pressure fitted the existence of a magnetic moment at $\mathrm{P}<42 \mathrm{GPa}$, a mixture of magnetic and nonmagnetic components at $42 \mathrm{GPa}<\mathrm{P}<59 \mathrm{GPa}$, and nonmagnetic only at $\mathrm{P}>59 \mathrm{GPa}$. In the compound $\mathrm{LaFeO}_{3}$ they found a mixture of metallic and gapped states between 70 and 120 $\mathrm{GPa}$, with the insulator-like resistivity below $70 \mathrm{GPa}$ and the metallic above $120 \mathrm{GPa}$. It has been found to be quite normal for the materials to pass through three distinct resistivity regimes as pressure progresses, and the resistivity changes to be accompanied by a magnetic transition.

\subsubsection{Conductivity as a function of Doping}

Doping a semiconductor to achieve a desired level of conductivity is a common and well-understood practice. The cuprates are a prime example of a material being doped. The undoped parent is a superb insulator. Doping can progress carrier by carrier from this insulating state right through to metallic. Few substances can be doped through such a large range of conductivities.

\subsubsection{Magnetic Breakdown as a function of Doping}

Perhaps less obvious is the impact of doping on magnetism, particularly in an antiferromagnetic material. Hole-doping introduces 
fundamental changes to the local spin and the long-range order. Removing the spin one-by-one changes the magnetism locally, then the local changes merge. Long-range order also begins to break down. Eventually, after a gradual transition as a function of the doping, the changes extend throughout the entire material and a new magnetic state has replaced the old.

Changing conductivity by doping changes spin density and arrangement. As described earlier, the hole doping not only changes conductivity, but in the cuprates is also an agent for breakdown of the antiferromagnetism by a progressive frustration mechanism. That is, the changes are not generally abrupt.

\subsubsection{Equivalence of Doping and Pressure}

The preceding illustrates the effects of pressure, and the coexistence of conductivity and magnetic transitions highlight the interwoven nature of the phenomena involved. There are indeed similarities in the effects of doping and pressure on the one hand, and a relationship between electrical conductivity and magnetism, or magnetic breakdown, on the other. Especially in Mott materials the magnetism and conductivity are intimately interwoven.

Generally, doping and pressure both cause concurrent change in magnetism and conductivity.

Pasternak and Taylor [72] refer to changes in band structure caused by the increased energy densities and changing relation between $\mathrm{W}$ (d-band width) and $U$ (on-site repulsion) under megabar pressures. 
The theoretical treatment of Suzuki and Otani [77] of solid oxygen under pressure obtained the result the pressure-induced MIT occurs because of band overlapping. Band overlapping is what happens when a degenerate semiconductor is doped into the regime of behaving as a metal.

Next we return to the topic of degenerate semiconductors to add detail from available knowledge. We will particularly notice p-type degenerate semiconductors, oxide semiconductors, and early superconductivity in ceramics.

\subsection{Degenerate Semiconductors (ii)}

The possibility of superconducting semiconductors was considered early e.g. [78]. The existence of such materials in reality was well confirmed in the 1960s with the observation of superconductivity in degenerate semiconductors such as $\mathrm{GeTe}, \mathrm{SnTe}, \mathrm{SrTiO}_{3-\mathrm{x}}$ and $\mathrm{Sr}_{1-\mathrm{y}} \mathrm{Ba}_{\mathrm{y}} \mathrm{TiO}_{3-\mathrm{x}}$ [corresponding refs in 79]; and in $\left(\mathrm{Ba}_{\mathrm{x}} \mathrm{Sr}_{1-\mathrm{x}}\right) \mathrm{TiO}_{3}$, and $\left(\mathrm{Ca}_{\mathrm{y}} \mathrm{Sr}_{1-\mathrm{y}}\right) \mathrm{TiO}_{3}$ [80]. All known such materials were strongly polar compounds with high static dielectric constant. Some are ferroelectric in the insulating state [80]. Non-polar semiconductors were considered incapable of superconductivity due to insufficient screening being available to the charge carriers [81].

"Encouraged" by the initially comparable results between polycrystals and single crystals, researchers were very soon studying the degenerate semiconductor superconductors in the ceramic form obtained from standard pressing and sintering [80]. 
From the dielectric properties of the materials it was believed $T_{C}$ varied according to changes in the symmetry or lattice parameter of the semiconductor [80], and that to cause variation of $T_{c}$ in those superconducting ceramics they needed to: either apply pressure, or

substitute ions, or change the oxygen stoichiometry (where possible). Superconductivity was a function of the changes.

The titanates are n-type, the tellurides p-type superconductors. he superconductivity was attributed to strong electron-phonon coupling with effective mass and dielectric properties playing an important role e.g. [79], [80], [82]. Crucially, they attempt to describe how $\mathrm{V}$ (the pairing strength of BCS theory in equation (2)) alters for an insulator-type material (rather than metallic), and in the presence of a high dielectric constant. In polar semiconductors the polar interaction of the background ions due to the added charge carriers is a very effective screen of the Coulomb interaction [79], [83]. At low frequencies the Coulomb interaction is well screened. When the dielectric constant falls at high frequencies the Coulomb interaction is enhanced. In a degenerate semiconductor the charge carriers themselves provide extra screening [84], as in metallic superconductivity. Once the material becomes ferroelectric, the dielectric constant drops markedly, and the Coulomb interaction is strengthened.

After the debut of the very first superconducting semiconductors more were hoped for, and it was thought a good place to look would 
be "pseudo-ferroelectrics" (dielectric constant increases with decreasing temperature without the material ever actually becoming

ferroelectric) [p 649, 82]. Through searching in this manner the number of degenerate semiconductors known to display superconductivity was extended.

Cohen [82] presents GeTe and SnTe as examples of p-type lowcarrier-density superconductors. He takes care to point out it is the p-type semiconductor that superconducts, and explains the superconductivity is a bulk characteristic of these tellurides. GeTe could be doped with (excess) tellurium, or with silver.

Much diligence was also put into demonstrating that the superconductivity in $\mathrm{SrTiO}_{3}$ is a bulk effect. The superconductivity of the n-type degenerate semiconductors traced out a dome shape. In the tellurides, the p-type superconductors, the superconductivity traced out the upward curve of the onset of a dome. Doping was not increased to be able to demonstrate the location of a maximum or the vanishing of superconductivity.

Strontium titanate was a particularly curious material, displaying so many interwoven complexities, that Cohen and others [p 654, 82] were inspired to say, "If $\mathrm{SrTiO}_{3}$ had magnetic properties, a complete study of this material would require a thorough knowledge of all of solid state physics."

Appel does consider the possibility and also does not find the presence of magnetic properties a barrier to superconductivity: "Finally, let us comment on magnetic impurities. Independent of whether or not magnetic impurities exist in $\mathrm{SrTiO}_{3-\mathrm{x}}$, we attribute superconductivity to the interaction between electrons and soft-optical phonons. Magnetic impurities will 
decrease $T_{c}$ and affect its $n$-dependence [ $n=$ number of electrons - the charge carriers]. Their effect becomes important if the transition temperature in the absence of these impurities, $T_{c o}$, becomes comparable with $\hbar / k_{B} \tau_{s}$ where $\tau_{s}$ is the spin lifetime. Both numbers are comparable for $\mathrm{n}=10^{20} \mathrm{~cm}^{-3}$ if $\tau_{\mathrm{s}} \approx 2 \times 10^{-11} \mathrm{~s}$. The mobility lifetime $\tau_{\mu} \approx 10^{-14} \mathrm{~s}$ at $4.2 \mathrm{~K}$. In general, $\tau_{\mathrm{s}}$ is roughly two orders of magnitude larger than $\tau_{\mu}$, so that the observed $T_{c}$ values may be affected by spinflip scattering". [84]

These days degenerate semiconductors are again of research interest, but without aims of superconductivity.

The iron pnictides were discovered by the Hosono Group, who specialise in wide band gap semiconductors. Being familiar with such matters, they recognise at room temperature the fluorine-free, or undoped, $\mathrm{La}\left(\mathrm{O}_{1-\mathrm{x}} \mathrm{F}_{\mathrm{x}}\right) \mathrm{FeAs}$ as a degenerate semiconductor - in the metallic state. [73]. With fluorine substitution (electron doping) this layered compound becomes superconducting up to $26 \mathrm{~K}$ and shows superconducting onset up to $32 \mathrm{~K}$.

Although the optically transparent semiconductors that interest the Hosono Group do not superconduct, we will take time to also make acquaintance with research on these materials.

\subsection{Optically Transparent Semiconductors}

The concept of see-through materials that can conduct electricity has intrigued the electronics industry with the potential commercial possibilities. Transparent $n$-type semiconductors were more readily available. With the continuing importance of the $p-n$ junction in electronic devices, motivation existed for the development of $p$-type 
transparent semiconductors, and these have become a speciality of the Hosono Group in Japan. However, p-type wide bandgap semiconductors are not easily encountered and even less so ones where the holes have mobility much beyond insulator level. Conductive oxides were of interest. They are "different" to the more customary semiconductors, such as silicon, seen in other applications. The first p-type TCO was NiO [85], announced in 1993. The conductivity of the material left a lot to be desired, but it was a breakthrough to have a real-life example. Very soon improvement followed, and now p-type TCOs have been developed to the level of incorporation into working devices. Important materials include $\mathrm{CuAlO}_{2}, \mathrm{SrCu}_{2} \mathrm{O}_{2}$, and $\mathrm{LaCuOCh}$ where $\mathrm{Ch}$ is one of the chalcogens $\mathrm{S}, \mathrm{Se}$ or Te.

From the point of view of electronics, the general problems in developing operative p-type TCOs, with mobility of the holes, can be summed up surprisingly briefly: The valence band edge of oxides is localised on the oxygen $2 p$ orbitals, separated by a wide gap from the cation conduction band. Holes are very localised, or "deeply trapped", with large effective masses. The result is little mobility of the charge carriers and difficulty in achieving doping. [86], [87]

Solutions to bypass the problems and increase the dispersion of the valence band were formulated. Kamiya and Hosono [86] list three ways this can be done:

1) by decreasing the nearest neighbour oxygen-oxygen distance, 2) by hybridization of cations with orbitals degenerate with the $O 2 p$ states,

3) by extending anion orbitals. 
Practically, these solutions can be implemented by:

1) applying pressure thus reducing the volume occupied by a given quantity of participating oxygen, or by doping more oxygen into the same volume,

2 ) using $d$ orbitals with energy levels similar to those of oxygen $2 p$. Copper is a prime candidate, and researchers of TCOs work with $\mathrm{Cu}^{+}$. The dispersive band thus produced has a lower effective mass [86] (and the closed shell of the $\mathrm{Cu} 3 \mathrm{~d}^{10}$ was not expected to result in optical absorption),

3) chalcogens were considered potentially useful e.g. [86].

"Good conduction" was seen in the oxychalcogenides of the form $\mathrm{LnCuOCh}$, where $\mathrm{Ln}$ is a lanthanide and $\mathrm{Ch}$ is a chalcogen. This material is layered and the low dimensionality works to produce a large band gap via suppressing band dispersions [86], in particular by suppressing the width of the conduction band [88]. $\mathrm{LaCuO}\left(\mathrm{S}_{1-\mathrm{x}} \mathrm{Se}_{\mathrm{x}}\right)$ demonstrates increasing hybridization with increasing $x(0 \leq x \leq 1)$, due to the wider spread of the Se $4 p$ orbitals compared to $S$, and hole mobility is increased [86]. The conductivity increases to degeneracy in LaCuOSe $(x=1)$ by doping $\mathrm{Mg}^{2+}$ onto $\mathrm{La}^{3+}$ sites [87].

The Hosono group is developing the use of functional nanospace and clathration to achieve desired conductive properties.

$\mathrm{SrTiO}_{3}$, and $\mathrm{BaTiO}_{3}$ have been shown to have a level of optical transparency [89]. Although researchers of $p$-type wide band gap semiconductors naturally stay with the $\mathrm{Cu}^{+}$that admirably suits the 
purposes of p-type optical transparency, there are similarities between the cuprous oxide TCOs and the cupric oxide HTS.

The Hosono Group came across $\mathrm{La}\left(\mathrm{O}_{1-\mathrm{x}} \mathrm{F}_{\mathrm{x}}\right) \mathrm{FeAs}$ and other new superconductors through research into new TCOs and other novel semiconductors [90]. 


\section{General Discussion}

At the beginning of the project the goal was to use dc magnetic susceptibility to confirm the location of the antibonding VHS in Bi2212 for comparisons with predictions made by Fermi liquid theory. In our preliminary view of this VHS there had also been another, anomalous, spike in the susceptibility in the extreme overdoped region along with a monotonic-style increase of the magnitude with doping. Neither of these phenomena has been predicted by theory and it was hoped to also investigate their existence. We did make headway with these goals. However, after becoming familiar with both collecting data from this experiment and the results of others, it became apparent the role of the VHS in the cuprates differs to the traditional view that it provides an increased DOS for an increased $\mathrm{T}_{\mathrm{C}}$ and that a change in perspective is needed for unravelling the mystery of superconductivity in the cuprates.

It was from the Fermi liquid approach that we knew where to look for the VHS and found its description of the location excellent. In the $\mathrm{Bi}$ 2212 the VHS is visible over such a restricted range of doping that exactitude is essential. Due to the monotonic increase in the susceptibility, a confirmed characteristic of the oxygen doped $\mathrm{Bi}$ 2212 , it is not possible to verify whether the Fermi liquid approach well describes the susceptibility curves as a function of temperature. So far, the monotonic increase is most likely to be related to the use of molecular oxygen as the dopant, and the shape of oxygen magnetic response is the same as that recorded for the BSCCO (e.g. compare Figures 11 and 16). From the VHS, the growth in 
magnitude can be as much as $10-15 \%$ after doping only a further 0.01 holes $/ \mathrm{Cu}$. Such a rise when the expectation is that values should be falling away again, implies the possibility the shape of the Bi-2212 curves may be dominated by the cause of this effect.

With the focus on the copper oxide layers there is a lack of information on the other layers of the cuprates. Experimental data indicates that oxygen doped cuprates have a magnetic response, increasing with doping, that is absent in non-oxygen doped cuprates. Many scientific fields have studied oxygen in many situations. Oxygen is consistently found to have a strong magnetic signature and is capable of many polymorphs. In the cuprates the doped-in oxygen does not enter the copper oxide layers. Instead, it is taken into what are known as the charge reservoir layers, the $\mathrm{BiO}$ layers in the case of the $\mathrm{Bi}-2212$. So far, the contribution of the BiO layer to the total magnetic data recorded for the BSCCO material, especially as oxygen uptake increases, has not been studied. This seems an oversight. This work shows lack of substantial oxygen, or charge reservoir layer, contribution to the recorded magnetic response of the HTS materials cannot be assumed.

As far as the anomalous spike at $T_{c} \approx 59 \mathrm{~K}$ is concerned, we are now satisfied it does not arise from experimental artefact. An explanation remains elusive, but results are included as future research into the far overdoped regime is likely to encounter consequences resulting from this characteristic. We do offer two possibilities for investigation:

- This second peak may result from splitting of the VHS itself, which could occur for example from possible changes to symmetry. Jahn- 
Teller effects, Mueller's motivation for exploring (and discovering) the first HTS [13], induce structural distortion [91]. It may be that such splitting is a signature of structural change and one might fruitfully explore this part of the phase diagram for evidence of symmetry change or structural distortion.

- Or, this too could be related to the use of oxygen as a dopant. Within the structure of the crystal, oxygen may possibly reach a critical packing density or level of chemical interaction with the copper oxide layers at the conditions that produce $T_{c} \approx 59 \mathrm{~K}$ on the overdoped side. A change between phases, or between different polymorphs, could show a similar peak in SQUID data, particularly when a magnetic change is also involved. [92]

Two other particularly notable results came out of the observation of the VHS:

1. Repeated passing through the VHS site has never given any indication that the peak in the DOS causes a rise in the $T_{c}$.

2. The VHS is rather more associated with the vanishing of superconductivity than with the maximum $T_{c}$.

The lack of increase in $T_{c}$ at the VHS may appear contradictory to the description of BCS theory and seem as support for proposals that require exotic physics or the invention of new states of matter to explain the superconductivity in the HTS. However, there are other possible explanations. It may simply be that the holes that participate in superconductivity do not 'see' the VHS at the zone boundary. This certainly seems to be the case in the underdoped region where superconductivity occurs only on the Fermi arcs near the zone diagonal e.g. [93], i.e. holes that pair may reside in parts of the 
Fermi surface away from the $(\pi, 0)$ zone boundary. There may also be other characteristics that can intensely affect the pairing mechanism, for example, screening strength.

Electronically the HTS materials are insulators that are doped to reach conductivity. We also know, electronically speaking, that:

1. A material with appropriate $2 \mathrm{D}$ characteristics will have a $2 \mathrm{D}$ VHS, which will mark a crossover between hole-like and electron-like conductivity

2. The best HTS have hole-like majority charge carriers.

3. In reaching a metallic state from an insulating state via doping, it is usual to pass through an intermediate semiconductor state, where at finite temperature electrical conductivity occurs before the gap completely closes. The situation can be different at $\mathrm{T}=0$ where an abrupt transition from the insulating to metallic states may be observed. See e.g. Fig. 33 above.

4. Such a metallic state is a 'degenerate semiconductor'.

5. It does appear to be the case that when $T_{c}$ is tracked with respect to $\rho$ superconductivity seems to be associated with the region near a MIT:

a) In granular aluminium, the electron-like pairs superconduct in the metallic region and superconductivity is lost on crossing the MIT to the insulator side.

b) In the cuprates, superconductivity appears in a dome shaped region about the MIT, as revealed by the Boebinger data (Fig. 33). 
6. The HTS cuprates have a temperature dependent MIT which shows the materials as metallic at higher temperatures. However, beneath this there is also the progression of a doping dependent MIT.

7. Charge pairing and charge mobility are not necessarily synonymous.

8. Superconductivity in ceramics is not new.

9. Superconductivity in semiconductors is not new.

As well as being doped Mott insulators the cuprates are also oxides. Rather than being naturally endowed with charge mobility, p-type oxides, even as semiconductors, characteristically have 'stuck' charge carriers. On the other hand, in polar semiconductors charge is strongly screened. In conventional, non-polar, semiconductors the metallic process of screening is weaker, too weak on its own to allow pairing, particularly to high temperatures e.g. [81]

Earlier it was noted the vertical, temperature dependent, MIT is affected by the horizontal, doping dependent, MIT. This too perhaps could benefit from an about turn in perspective. The horizontal doping dependent insulator to metal movement of a degenerate semiconductor can be hidden by the vertical temperature dependent Mott MIT. At a suitable temperature a material with a temperature dependent MIT will naturally present as metallic. The cooler, insulator, side of the transition may still at the same time be slowly progressing towards metallic via a doping dependent MIT.

This appears to be happening in the cuprates, with further concealment from the superconducting dome which obscures the 
nature of the normal state. Above the dome, at warmer temperatures, the material is metallic - as appropriate to a temperature dependent MIT. To the right of the dome, at high doping, the material is also metallic - as appropriate to a doping dependent MIT. At lower dopings and temperatures the material is superconducting and the normal state extremely difficult to access even for a momentary glimpse. To add to the confusion, a semiconductor doped to an extreme level of metallicity looks very much like an intrinsic metal. Now we have information that under the dome there is indeed a regular progression from the fully insulating state towards the metallic state.

Witte [94] recalls the use of polarizability (Herzfeld criterion) to describe in general the valence electron density dependency through a MIT. The valence electrons and atomic ions have an atomic polarizability that increases with decreasing radius until it finally diverges, also known as "dielectric catastrophe". When this happens the electrons can be considered to become delocalized and conduction therefore becomes metallic. Herzfeld [95] relates the dielectric constant, refractivity and transparency in explaining the concept more fully.

Thirteen years after the discovery of the cuprates Edwards et al. [96] express the relationship in terms of charge carrier concentration and effective Bohr radius (of the localised carrier). They compare various cuprates known at the time with common semiconductors. While they note there are "important differences" to conventional semiconductors, such as the presence of antiferromagnetism and significantly higher static dielectric constants, they nonetheless “...propose that High $\mathrm{T}_{\mathrm{C}}$ layered cuprates are doped semiconductors, irrespective 
of the level of doping." and find that in common with the conventional semiconductors, properties such as electrical conductivity are dominated on the insulator side of the (doping dependent) MIT by "...thermally-assisted hopping (and tunnelling) of excess charge carriers between localized states."

It is interesting that the superconducting ability in Mott insulators was predicted [97] thirteen years before the HTS announcement of Bednorz and Müller by using similar concepts. The treatment was inspired by organic compounds, not the cuprates of the time, but functioned also on considering the material as a Mott semiconductor rather than a Mott insulator. Polarisation was important, and in the favourable circumstances, such a Mott material was found to fall into either a superconducting or a ferroelectric state, but could not be both at the same time.

Research on the iron-based HTS is still in its infancy but preliminary works find large polarizability [98], and in particular that the resultant screening is likely to be strong.

In 2006 a number of HTS researchers provided comments to Nature Physics [99] about the state and future of the field:

"Remarkably the superconductivity itself seems among the least mysterious of the various phenomena in the copper oxides. ..." T. Senthil makes a comment reflecting a widespread viewpoint.

Masatoshi Imada wonders why "No theory has been able to explain why most of the doped or metallized antiferromagnetic insulators such as $\mathrm{LaTiO}_{3}$, $\mathrm{V}_{2} \mathrm{O}_{3}, \mathrm{NiS}_{2}$ and $\mathrm{Sr}_{2} \mathrm{VO}_{4}$ do not show superconductivity even at very low temperatures." If a magnetic pairing mechanism were to be the cause 
of the superconductivity in the cuprates it is natural to expect one may find superconductivity in other materials with similar magnetic properties. Theory is deliberately not our consideration at this stage, but from even the briefest of looks at physical characteristics we can begin to see that maybe we could have expected these compounds to not superconduct. $\mathrm{LaTiO}_{3}$ is not only antiferromagnetic but also a relative of $\mathrm{SrTiO}_{3}$ which does superconduct when doped. First, we recall $\mathrm{SrTiO}_{3}$ and the other superconducting titanates are n-type.

Then, that 'good' p-type conductivity in oxides is remarkably difficult to achieve. $\mathrm{LaTiO}_{3}$ is p-type [100]. Copper is used to support p-type conductivity, titanium supports the n-type. When enough oxygen is doped into $\mathrm{LaTiO}_{3}$ it does become n-type - metallic - and at the same time falls into ferroelectric ordering [101]. Similarly, $\mathrm{V}_{2} \mathrm{O}_{3}$ [102] and $\mathrm{NiS}_{2}$ [103] are p-type materials, not n-type, and have the concomitant limitations. Detail on $\mathrm{Sr}_{2} \mathrm{VO}_{4}$ was not found.

Matthias Vojta notes interest in the HTS materials arose simply because of the superconductivity:

"Why is $T_{c}$ so high and how can we make it higher? That is probably the ultimate question in the context of high- $T_{c}$ superconductivity. ..."

and in this context realises...

"But do we need to understand these phenomena [e.g. stripes/competing normal state phenomena] to understand high- $\mathrm{T}_{\mathrm{c}}$ superconductivity?.....Is it mandatory to understand the pseudogap in order to design materials with higher $\mathrm{T}_{\mathrm{c}}$ ?

... What level of understanding is needed to solve the high- $\mathrm{T}_{\mathrm{c}}$ problem?

...Progress will only be made by discerning primary from secondary effects."

The distinction between understanding the cuprates and understanding their superconductivity may be important. 
Superconductivity has three basic requirements:

1. screening of the Coulomb interaction

2. a mechanism that makes the charge carriers join as a pair

3. once pairs have formed they need to have mobility.

P.W. Anderson said two decades before the HTS discovery [104], "Once bound the pairs must also move:" In the initial search for superconductivity in semiconductors Ge was tested and found to remain in the normal state right down to very low temperatures. Cohen commented [82], "These results were not surprising to most people, since at such low temperatures, there are very few free electrons in this system, and it could be argued that this material was not even a conductor let alone a superconductor." and notes that generally for superconductivity to develop in a semiconductor, "A maximization of the carrier concentration $n$ is essential."

In other words, the charge mobility comes from the fermions, not from the bosonic pairing. - Recall the detection of pairs in an insulator (Stewart et al. [20]).

All three superconductivity requirements are in metals conceptually satisfied by the same source. With a very high density of extremely mobile electrons there is a good chance of frequent local excesses of negative charge. Consequently, screening can result. The unusual would be for the electrons to be insensitive to the lattice phonons, therefore when they can approach each other closely enough exchanging phonons becomes viable. The screening, pairing and mobility requirements all arise essentially from characteristics of the two electrons that form a pair. 
In the HTS, or in a semiconductor, fulfilment of each of the three superconductivity requirements may be more broadly sourced. This does not necessarily require unusual phenomena. It can however, lead to complications. For example different mechanisms, such as those driving mobility and pairing, can vary differently as a function of temperature and other characteristics.

Are phonons essential to the pairing mechanism in the cuprates? Many believe so e.g. [68], [105], [106], [107]. However, the cuprates appear to become superconducting out of a semiconductor state. Cohen recalls "In the case of a polar semiconductor, a doped ferroelectric, or a doped pseudo-ferroelectric (i.e., SrTiO3) the possibility of strongly coupling electrons to optical phonons arises." [p 637, 82]. The term "phoplasmon" was coined for the resulting coupling of the phonon modes and the plasma modes in the many valley model. BCS theory was formulated for superconductivity resulting out of metallic conduction. While the final establishment of a theory of semiconductor superconductivity appears to have never eventuated, the key concept that in a semiconductor coupling of modes can be expected is another factor that can lead to difficulties in distinguishing cause, consequence and coincidence.

Furthermore, a recurring theme in non-metallic superconductivity is incompatibility of the superconductivity and ferroelectricity. Although this is a concept yet to be studied and confirmed, it leads to intriguing possibilities regarding the status of magnetic ordering in the HTS. Firstly, with the enormous upper critical fields of the HTS materials it is feasible some degree of internal magnetism be possible before superconductivity is destroyed. Secondly, antiferromagnetic ordering is particularly suitable to coexist with 
superconductivity, as the bulk field is zero and the effect felt locally thus much reduced - compared to say, ferromagnetism. However, any remnant of magnetic order may be sufficient to deter ferroelectric tendencies and help cause a material to fall into the superconducting state. Multiferroic materials are rare and require specific and stringent conditions to exist [108]. When a material is not a multiferroic, the presence of magnetism automatically ensures protection against ferroelectricity. In this case, such a possible contribution would not even supply one of the three requirements for superconductivity - it would allow the environment for (polar) screening to function. More directly related to superconductivity, we know from geophysics that changes in magnetism can boost conductivity. In other words, effective charge mobility is improved. Local fluctuations in antiferromagnetic ordering may also aid superconductivity in this manner.

In the case of the cuprates the copper too, can protect against ferroelectricity, thus adding to possible reasons the cuprates were the first HTS found. The filled shell of the copper d-orbitals that makes it a particularly able participant in the semiconductor oxides, is also a characteristic that precludes the formation of ferroelctricity.

In a separate vein, the antiferromagnetism is a characteristic of Mott insulators. That is, it is can signal that the material has a wide gap. In addition, the multiple valence states of the transition metals makes them prime candidates for being able to dope to an extended concentration of charge carrier. 
At the time of the discovery of superconductivity in the cuprates the HTS displayed other characteristics that were unfamiliar and poorly understood.

1. 2D conductivity, with the consequent VHS.

2. Antiferromagnetic Mott insulators.

3. Strong correlation.

Each one of these concepts was in existence long before the HTS, however, the physics underpinning each one was not well understood.

To the list we could now add

4. (p-type) wide gap semiconductors.

5. Antiferromagnetic semiconductors.

There was also a lack of satisfactory description of transition metals and their related compounds and the name "cuprate" has detracted attention from the fact they are also oxides. As noted by the Hosono Group, oxide semiconductors are "different".

Particularly in the case of the VHS that results from 2D geometry, physical understanding of the phenomenon appears to have grown as a direct result of its appearance in the HTS. This makes even more difficult the task of differentiating which phenomena are causing superconductivity, which are supportive of it existence, which are indifferent, and which are detrimental. Or even, as may be the case with magnetism, which can give on the one hand and take with the other, so requiring an even more refined viewpoint.

As our oxygen information has come from biology and medicine inspired research, so has our knowledge of any pressure-doping 
equivalence come from geophysics, whose major interest is naturally iron compounds. Nevertheless, important information is available about underlying physics relevant to evaluating the superconductivity of the HTS materials. As well as demonstrating a strong link between magnetic transitions and electrical conductivity, geophysics study of the pressure-induced MIT in transition metal compounds shows us it can indeed make a difference whether the transition metal is odd- or even-valence. Like other areas, the earth sciences also note Mott insulators are wide band gap materials. We have found important past and present information on semiconductors that leads to further understanding of the HTS and there are even clues from the first generation superconductors.

We find that the ability to form pairs, the ability to provide an environment where the pairing mechanism may function, and the ability of the pair to move once formed may function as distinct considerations. Many areas of research, perhaps seemingly unrelated in the first instance, have contributed to our view. Overall, the superconducting HTS materials are found to function as semiconductors, not as metals.

To start to build detail gleaned from such a wide base, some suggestions are made for ongoing research that could benefit understanding of superconductivity and lead to the design of further materials:

- Measure the contribution to magnetic susceptibility data from oxygen doped into the charge reservoir layers. In the first instance comparisons of strontium doped LSCO with oxygen doped LSCO would be a guide. 
- Evaluate the cuprates as semiconductors.

- Repeat high pressure studies on magnetic-conductivity transitions using $\mathrm{CuO}$ and undoped cuprates.

- It would be instructive to suppress the superconductivity as far as possible past the edge of the dome for other HTS to try to confirm normal state resistivity characteristics of a variety of Mott-based superconductors.

- It may also aid superconductivity understanding to measure $T_{c}$ as a function of resistivity for superconducting elements as pressure is increased.

- To the multiferroic community, an assessment of the extent of the incompatibility of superconductivity and ferroelectricity could be useful.

- Evaluate what changes happen when the chalcogens Se and $S$ are used in cuprates, particularly LSCO which is most directly related to the TCOs.

- Directly compare further differences and similarities between the cuprous TCOs and the cupric HTS.

- Locate the VHS in the magnetic susceptibility for other cuprates and HTS. Particularly from the point of view of confirming whether it is a general characteristic the VHS (change of charge carrier) remains closer to the vanishing of superconductivity than the maximum $T_{c}$.

People who first looked for superconductivity in semiconductors were, " ... motivated by the idea that semiconductors and semimetals could be useful systems for studying superconductivity. This is primarily because these materials are better understood than metals and it is possible to make large changes in the carrier densities and band structures (through alloying and 
pressure) to study the dependence of the superconducting properties on the normal-state properties." [p. 617, 82]

Now we find the second generation superconductors are semiconductors themselves.

\section{Conclusion}

While a global understanding of the cuprates must include superconductivity, the converse is not necessarily true - to understand the cause of superconductivity in the cuprates, details of simultaneously occurring phenomena do not need to be included, albeit the long-term goal would be to understand the effects such phenomena have on ability to superconduct.

The intense interest in the HTS arose purely out of their ability to carry electrical current without energy loss. We can make the task of understanding this ability much easier by not assuming we must do everything at once. What ignites the pairing of two electrons does not have to involve all phenomena present in a material. Moreover, we should recognise there may exist phenomena that support the existence of a pair while yet being completely unable to cause the pair to form. 
As more and more materials and composites are found capable of superconductivity there will inevitably be a continually growing variation in the kinds, combinations and intensities of phenomena that are displayed in conjunction with the superconductivity. We should ask ourselves how often we realistically need an entirely different mechanism to explain the appearance of a phenomenon in yet another unfamiliar material, and when we would do better to concentrate on separating unfamiliar phenomena from the fundamental nature of electrical conductivity and on how a new phenomenon may affect a known superconductivity mechanism.

We must also be prepared for the eventuality that the more correlated a material is, the more cause and effect can become subjective. Agents like pressure or doping cause concurrent conductivity and magnetic transitions. As well as the transitions occurring concurrently, both magnetism and conductivity depend on charge. As a result they are highly interwoven and to extract which is dependent on which can be, in many respects, an academic exercise. In addition, in cases where mobility, screening and pairing are controlled by different phenomena there is ample opportunity for prolonged debate over what is the cause of the superconductivity. Through a variety of experiences and samples the antibonding VHS of the $\mathrm{Bi}-2212$ remains evident. However, the magnetic susceptibility data continues to be dominated by a strong monotonic rise with increasing doping. Before embarking on detailed study of the overdoped regime it would be prudent to first gain understanding of the behaviour, and therefore the contribution, of the quantity of oxygen that is inserted into the HTS to reach such levels of doping. 
The magnetic susceptibility data also shows an extra peak close to the VHS on the still further overdoped side (at $\mathrm{T}_{\mathrm{c}} \sim 59 \mathrm{~K}$ ) of the $\mathrm{Bi}(\mathrm{Pb})-2212$. Splitting of the VHS itself is a plausible explanation. However, the data so far may be consistent with this secondary peak being due to the oxygen dopant.

The cuprates contain a crystal structure of a (genuine) 2D nature. Therefore they have a 2D VHS. No evidence has been found to support the idea of the VHS in the cuprates being able to cause superconductivity, in particular of being behind the pairing mechanism. (This would require e.g. structural fluctuations of some sort associated with the proximate VHS). To the contrary, it rather more seems the 2D VHS is associated with disappearing superconductivity as the majority charge carrier switches. Many normal state properties of the cuprates can be accounted for by the presence of the 2D VHS, a phenomenon that occurs naturally when a system shows appropriate $2 \mathrm{D}$ characteristics. A material with a $2 \mathrm{D}$ VHS will have certain electronic properties, such as a peak in the DOS in the middle of a band and a ground state instability in the Fermi surface when the Fermi energy equals the VHS energy.

Studies on the HTS cuprates, the superconducting semiconductors of the 1960s, the copper-based p-type wide gap transparent semiconductors, the iron-based superconductors, and geophysics work on the Mott transition show a relationship between widegapped conductivity and the ability to superconduct. Low dimensionality has been found an effective manner of achieving large gaps. The VHS could be an incidental side effect in the known 
HTS, able to affect superconductivity simply because both phenomena occur together in the same material, but neither fundamentally being cause or consequence of the other.

Some complete changes in perspective are found very useful, even essential. We find it greatly beneficial to regard the 'copper based' HTS as oxides rather than cuprates. By considering doping to have a pressure-like effect, instead of pressure to be equivalent to doping a wealth of information on the MIT of transition metal compounds is opened to us. Above all, we have found the cuprates are better regarded as semiconductors than as metals, particularly in the superconducting regime, and it is the underlying wide gap semiconductor that is the superconductor.

As Imada notes, a theory for a magnetic pairing mechanism in the cuprates should not only explain why such a mechanism functions in these materials, but also explain why it does not function in the noncuprate materials with very similar magnetic properties that do not superconduct even a very low temperatures. Even lacking confirmation of the nature of the pairing mechanism, consideration of such non-cuprate magnetic materials [e.g. doped $\mathrm{LaTiO}_{3}$ ] as semiconductors is able to illustrate why there should be a difference in expectation of superconductivity between otherwise similiar materials. With regard to the copper- and iron- based HTS, the transition metal antiferromagnetic insulators are natural wide gap materials, - with the added bonus of being able to be doped extensively. They also naturally avoid becoming ferroelectric. 
As expected we find the antibonding VHS to be an enduring feature of the phase diagram and found its location superbly described by the use of Fermi liquid theory. However, the dominant role of the DOS in the production of the $T_{c}$ comes from a description of superconductivity specifically formulated for metallic conductivity. Phonons remain strong contenders for playing an essential role in the HTS superconductivity, but screening, pairing and charge mobility in the HTS may result from different sources. For example, electron-electron interaction remains likely to play an important role in the HTS. Knowing what is the pairing mechanism and knowing what are the causes of the high temperatures of the superconductivity are not necessarily the same questions.

As low-dimensionality has been found so effective and practical in achieving functioning wide gap p-type conductivity, we may nonetheless have the 2D VHS with us for a while. However, there are many potential methods available to develop semiconductors to have the attributes that lead to superconductivity in the cuprate - or oxide - HTS materials. Preliminary information suggests similar attributes are behind the HTS generally. With the growing ability to design novel semiconductors we find there are many possibilities ahead of us for the development of superconductivity. 


\section{References}

[1] Léon Van Hove, The Occurrence of Singularities in the Elastic Frequency Distribution of a Crystal, Physical Review, 89, No. 6, 1189 (March 6 1953)

[2] Van Hove singularity in Raman scattering spectra of high-Tc superconductors O.V. Misochko and E. Ya. Sherman, Phys. Solid State 40 (1), January 1998.

[3] A. Kaminski, S. Rosen Kranz, H.M. Fretwell, M.R. Norman, M. Randeria, J.C. Campuzano, J-M. Park, Z.Z. Li. And H. Raffy, Change of Fermi-surface topology in $\mathrm{Bi}_{2} \mathrm{Sr}_{2} \mathrm{CaCu}_{2} \mathrm{O}_{8+\delta}$ with doping, Physical Review B 73, 174511 (2006)

[4] G. Triscone, J.-Y. Genoud, T. Graf and J. Muller, Variation of the superconducting properties of $\mathrm{Bi}_{2} \mathrm{Sr}_{2} \mathrm{CaCu}_{2} \mathrm{O}_{8+\delta}$ with oxygen content, Physica C. 176, issues 1-3, 247 (1991)

[5] Cristina Buzea and Kevin Robbie, Assembling the puzzle of superconducting elements: a review, Supercond. Sci. Technol. 18, R1-R8 (2005), IOP Publishing

[6] Adrian Cho, Magnetic Measurements Hint at Toastier Superconductivity, Science 32042 (4 April 2008)

[7] N. Manyala, J. F. DiTusa, G. Aeppli \& A. P. Ramirez, Doping a semiconductor to create an unconventional metal, Nature 454, (21 August 2008) 
[8] N. F. Mott, The Basis of the Electron Theory of Metals, with Special Reference to the Transition Metals, Proc. Phys. Soc. A 62, no. 7, p 416 (1949)

[9] Michael P. Marder, pp 659-661, Condensed Matter Physics, John Wiley \& Sons, Inc, New York 2000.

[10] J. L. Tallon, C. Bernard, G.V.M. Williams, and J.W. Loram, Zninduced $T_{c}$ Reduction in High- $T_{c}$ Superconductors: Scattering in the Presence of a Pseudogap, Physical Review Letters 79, No.26 (29 December 1997)

[11] A. Pirou, Y. Fasano, E. Giannini and Ø. Fischer, Effect of oxygen-doping on $\mathrm{Bi}_{2} \mathrm{Sr}_{2} \mathrm{Ca}_{2} \mathrm{Cu}_{3} \mathrm{O}_{10+\delta}$ vortex matter: Crossover from electromagnetic to Josephson interlayer coupling. arXiv:0802.2617v1 [cond-mat.supr-con].,

[12] N.W. Ashcroft, Fluctuation and higher temperature superconductivity in lighter element systems, Physica C 468, 115125 (2008)

[13] J. Georg Bednorz and K. Alex Müller, Perovskite-Type Oxides The New Approach to High- $T_{c}$ Superconductivity, Nobel Lecture 8 December 1987, Part Two: Properties of the New Superconductors, http://nobelprize.org/nobel prizes/physics/laureates/1987/bednorzmuller-lecture.pdf 
[14] S. Uchida, Critical points in the cuprate phase diagram, Solid State Communications 126, 57-61 (2003) [15] J.A. Slezak, Jinho Lee, M. Wang, K. McElroy, K. Fujita, B.M. Andersen, P.J. Hirschfeld, H. Eisaki, S. Uchida and J. C. Davis, Imaging the impact on cuprate superconductivity of varying the interatomic distance within individual crystal unit cells. PNAC 105, No. 9 3203-3208 (4 March 2008)

[16] http://physicsworld.com/cws/article/print/772/1/pw-13-02-08fig1 a website of IOP Publishing.

[17] Maurice Rice is at the Institute for Theoretical Physics, ETH Zürich, Switzerland The quoted comment was made Dec 61999 http://physicsworld.com/cws/article/print/840

[18] Leon N. Cooper, Bound Electron Pairs in a Degenerate Fermi Gas Physical Review 104, No. 4 (November 15 1956)

[19] J. Bardeen, L. N. Cooper, and J. R. Schrieffer, Phys. Rev. 108, 1175 (1957)

[20] M.D. Stewart Jr., Aijun Yin, J. M. Xu, James M. Valles Jr., Superconducting Pair Correlations in an Amorphous Insulating Nanohoneycomb Film, Science, 318, 1273 (23 November 2007)

[21] Introduction to the ARPES method by the Leibniz Institute for Solid State and Materials Research, Dresden http://www.ifw-dresden.de/institutes/iff/research/SC/arpes/method 
[22] D. Pavuna, I. Vobornik and G. Margaritondo, Photoemission Experiments on High-Tc Superconductors: Recent Progress and Some Open Questions, J. Supercond. Novel Magn. 13, No. 5, p. 749 (October 2000)

[23] J. G. Storey, Electronic Structure and Thermodynamic properties of High Temperature Superconductors, PhD thesis, Victoria University of Wellington 2008

[24] Neil W. Ashcroft and N. David Mermin, Susceptibility of metals: Pauli Paramagnetism, pp 661-665, Solid State Physics, Holt, Rinehart and Winston, New York 1976

[25] J.G. Storey, J.L. Tallon, G.V.M. Williams, Thermodynamic properties of $\mathrm{Bi}_{2} \mathrm{Sr}_{2} \mathrm{CaCu}_{2} \mathrm{O}_{8}$ calculated from the electronic dispersion, arXiv:0704.2432v1 [cond-mat.supr-con]

[26] R.S. Markiewicz, A Survey of the Van Hove Scenario for High$T_{c}$ Superconductivity with Special Emphasis on Pseudogaps and Striped Phases, J. Phys. Chem. Solids Vol. 58, No. 8, pp. 1179-1310 (1997)

[27] D.M. King, Z.-X. Shen, D.S. Dessau, D.S. Marshall, C.H. Park, W.E. Spicer, J.L. Peng, Z.Y. Li, and R.L. Greene, Observation of a Saddle-Point Singularity in $\mathrm{Bi}_{2}\left(\mathrm{Sr}_{0.97} \mathrm{Pr}_{0.03}\right)_{2} \mathrm{CuO}_{6+\delta}$ and lts Implications for Normal and Superconducting State Properties, Physical Review Letters 73, No. 24 (12 December 1994) 
[28] Private communication with Dr. James Storey. Presentations of theoretical predictions for the VHS.

[29] G. Litak, Van Hove Singularity and Superconductivity in a Disordered Hubbard Model, phys. stat. sol. (b) 229, No. 3, 14271449 (2002)

[30] J. Bok, J. Bouvier, High Tc Superconductors: the van Hove scenario, a review, Physica C 282-287, pp 294-298 (1997)

[31] S. Takács, Dependence of the critical temperature and energy gap of superconductors on a singularity in the density of states, Physical Review B 48, No 17, (1 November 1993-I)

[32] Xu Tiefeng and Bai Guiru, On the enhancement of superconductivity due to Van Hove singularity, Zeitschrift für Physik B - Condensed Matter 89, 35-37 (1992)

[33] Guy Deutscher, New Superconductors : From Granular to High $T c$, World Scientific Publishing Co. Pte. Ltd., p. xxiii, Singapore (2006)

[34] R.C. Dynes and J.P. Garno, Metal-Insulator Transition in Granular Aluminium, Physical Review Letters 46, No2 p137 (12 January 1981)

[35] N. F. Mott, Metal-Insulator Transition, Reviews of Modern Physics, Vol. 40, No. 4 pp677-683 (October 1968) 
[36] M.W. Haverkort, Z. Hu, A. Tanaka, S.V. Streltsov, M.A. Korotin, V.I. Anisimov, H.H. Hsieh, H.-J. Lin, C.T. Chen, D.I. Khomshii, and L.H.Tjeng, Orbital Assisted Metal-Insulator Transition in $\mathrm{VO}_{2}$, PRL 95, 196404 (2005)

[37] S. V. Kravchenko, Metal-Insulator Transition in Two Dimensions, Brazilian Journal of Physics, 29, No. 4 (December 1999)

[38] M.Y. Simmons, A.R. Hamilton, M. Pepper, E.H. Linfield, P.D. Rose and D.A. Ritchie, Metal-Insulator Transition at $B=0$ in a Dilute Two Dimensional GaAs-AlGaAs Hole Gas, Physical Review Letters, 80, No.6 (9 February 1998)

[39] Deutscher, G. The High $T_{c}$ Cuprates as Nanoscale Inhomogeneous Superconductors (2007) in : High $\mathrm{T}_{\mathrm{c}}$ Superconductors and Related Transition Metal Oxides, BussmannHolder A. And Keller H. (Eds.), Springer-Verlag, Berlin Heidelberg

[40] Ashkenazi, J.; Eremin, Mikhail V.; Cohn, Joshua L.; Eremin, Ilya; Manske, Dirk; Pavuna, Davor; Zuo, Fulin New Challenges in Superconductivity: Experimental Advances and Emerging Theories Proceedings of the NATO Advanced Research Workshop, held in Miami, Florida, 11-14 January 2004, Edited by J. Ashkenazi, M.V. Eremin, J.L. Cohn, I. Eremin, D. Manske, D. Pavuna, and F. Zuo. Berlin: Springer, 2005.

Via http://adsabs.harvard.edu/abs/2005ncse.conf.....A 
[41] Mark Rubenstein, Investigation of the Metal-Insulator Transition in $\mathrm{V}_{2} \mathrm{O}_{3}$ by Nuclear Magnetic Resonance, Phys. Rev. B. 2, No. 12 (15 December 1970)

[42] Elbio Dagotto, Complexity in Strongly Correlated Electronic Systems, Science 309, 257-262 (8 July 2005)

[43] T. Stoto, D. Pooke, L. Forro and K. Kishio, Oxygen distribution, incommensurate modulation, and structural disorder in $\mathrm{Bi}_{2} \mathrm{Sr}_{2} \mathrm{Ca}_{1-y} \mathrm{Y}_{y} \mathrm{Cu}_{2} \mathrm{O}_{8+\delta}$ and $\mathrm{Bi}_{10} \mathrm{Sr}_{15} \mathrm{Fe}_{10} \mathrm{O}_{46}$ single crystals, Physical Review B 54, No. 22 (1 December 1996-II)

[44] J. B. Forsyth, P.J. Brown and B. M. Wanklyn, Magnetism in cupric oxide, J. Phys. C: Solid State Phys. 21 (1988) 2917-2929, IOP Publishing

[45] M.I. Petrov, D.A. Balaev, K.A. Shă̌khutdinov, and B.P. Khrustalev, Effect of thermal fluctuations on the resistive properties of HTSC+CuO composites, Phys. Solid State 39 No. 11 (1997)

[46] Federico Gorelli, Mario Santoro, Roberto Bini, and Lorenzo Ulivi Extended infrared adsorption spectroscopy study of the magnetic properties of solid oxygen at high-pressure and low-temperature. Physical Review B 77, 132103 (2008) (11 April)

[47] Yu.A. Freiman and H.J. Jodl, Solid oxygen, Physics Reports, vol. 401, pp. 1-228 (2004) 
[48] Hirofumi Kanoh and Katsumi Kaneko. Magnetic Spin States of $\mathrm{O}_{2}$ Confined in a Graphitic Slit-Shaped Nanospace at Low Temperature J. Phys. Chem. 100, 755-759 (1996)

[49] Ryo Kitaura, Susumu Kitagawa, Yoshiki Kubota, Tatsuo C. Kobayashi, Koichi Kindo, Yoshimi Mita, Akira Matsuo, Michihiro Kobayashi, Ho-Chol Chang, Tadashi C. Ozawa, Megumi Suzuki, Makoto Sakata, Masaki Takata, Formation of a One-Dimensional Array of Oxygen in a Microporous Metal-Organic Solid, Science 298, no. 5602, pp. 2358 - 2361, (20 December 2002)

[50] N. R. Grey, and L.A.K. Staveley, Thermodynamic properties of clathrates, Molecular Physics 7, Iss. 1, 83-95 (1964)

[51] S. Bandow, T. Yamaguchi, and S. ljima, Magnetism of adsorbed oxygen on carbon nanohorns, Chemical Physical Letters 401, 280-384 (2005)

[52] U Köbler and R Marx, Susceptibility study of physisorbed oxygen layers on graphite ,Phys Rev B 35, No 18 (15 June 1987)

[53] Susumu Kitagawa, Ryotaro Matsuda Chemistry of coordination space of porous coordination polymers, Coordination Chemistry Reviews 251, 2490-2509 (2007)

[54] Magnetic Susceptibilities of the Elements and Inorganic Compounds, Section 4, CRC Handbook of Chemistry and Physics, 90 $0^{\text {th }}$ Edition, CRC Press/Taylor and Francis 2009 (Internet version 2010 http://www.hbcpnetbase.com/) 
[55] V.I. Danilov, V.M. Drobin, V.N. Trofimov, Ferromagnetic Behaviour of non-magnetic samples, phys. stat. sol. (a) 98, K137 (1986)

[56] D. G. LeGrand, Detection of Crystalline Phase Transition by Magnetic Susceptibility, Journal of Polymer Science 60, Issue 170 (1962)

[57] M.R. Presland, J.L. Tallon, R.G. Buckley, R.S. Liu and. N.E. Flower, General trends in oxygen stoichiometry effects on $T_{c}$ in $B i$ and TI superconductors, Physica C 176, 95 (1991)

[58] A. Avella, F. Manchini and D. Villani, The overdoped regime in $\mathrm{La}_{2-x} \mathrm{Sr}_{x} \mathrm{CuO}_{4}$, Sol. State Commun. 108, No. 10, pp723-725 (1998)

[59] J.W. Loram, K.A. Mirza, J.R.Cooper, N. Athanassopoulou and W.Y. Liang, Thermodynamic Evidence on the Superconducting and Normal State Energy Gaps in $\mathrm{La}_{2-x} \mathrm{Sr}_{x} \mathrm{CuO}_{4}$ Proceedings of 10th Anniversary HTS workshop, World Scientific, Singapore, 1996 (p. 341)

[60] F.J. Ohkawa, Anisotropic renormalisation of quasiparticles in cuprate-oxide superconductors, Physica C 301, 246-254 (1998)

[64] G.S. Boebinger, Yoichi Ando, A. Passner, T. Kimura, M. Okuya, J Shimoyama, K. Kishio, K. Tamasaku, N. Ichikawa, S. Uchida, Insulator-to-Metal Crossover in the Normal State of $\mathrm{La}_{2-x} \mathrm{Sr}_{x} \mathrm{CuO}_{4}$ Near Optimal Doping, Physical Review Letters 77, No. 27, (30 December 1996) 
[65] C. Castellani, C. Di Castro and M. Grilli, Comment on "Insulator-to-Metal Crossover in the Normal State of $\mathrm{La}_{2-x} \mathrm{Sr}_{x} \mathrm{CuO}_{4}$ Near Optimum Doping", arXiv:cond-mat/9709278v1 [cond-mat.suprcon]

[66] S. Ichikawa and I. Terasaki, Metal-insulator transition in $\mathrm{Ca}_{1-x} \mathrm{Li}_{x} \mathrm{Pd}_{3} \mathrm{O}_{4}$, PRB 68, 233101 (2003)

[67] P. Fournier, P. Mohanty, E. Maiser, S. Darzens, T. Venkatesan, C.J. Lobb, C. Czjzek, R.A. Webb, and R.L. Greene, Insulator-Metal Crossover near Optimal Doping in $\mathrm{Pr}_{2-x} \mathrm{Ce}_{x} \mathrm{CuO}_{4}$ : Anomalous Normal-State Low Temperature Resistivity, Physical Review Letters 81, No. 21, (23 November 1998)

[68] Karol Fijalkowski and Wojciech Grochala, The 'magic' electronic state of high-Tc cuprate superconductors, Dalton Tansactions, pp 5447-5453 (advance article on the web 21 July 2008) DOI: $10.1039 / \mathrm{b} 802879 \mathrm{fww}$ w.rsc.org/dalton

[69] J.L. Tallon, J.W. Loram, The doping dependence of $T^{*}$ - what is the real high- $T_{c}$ phase diagram?, Physica C 349, 53-68 (2001)

[70] Kenneth Barbalace http://klbprouctions.com/. Periodic Table of Elements - Sorted by Electrical Conductivity.

EnvironmentalChemistry.com. 1995 - 2009.

http://environmentalchemistry.com/yogi/periodic/electrical.html data from update of 22 February 2007 
[71] Yoichi Kamihara, Takumi Watanabe, Masahiro Hirano, and Hideo Hosono, Iron-Based Layered Superconductor $\mathrm{La}\left[\mathrm{O}_{1-\mathrm{x}} \mathrm{F}_{\mathrm{x}}\right] \mathrm{FeAs}$ $(x=0.05-0.12)$ with $T_{c}=26 \mathrm{~K}$, Journal of the American Chemical Society 130, pp 3296-3297 (2008)

[72] M. P. Pasternak and R. D. Taylor, Pressure-Induced Metallization and Electronic-Magnetic Properties of Some Mott Insulators, phys. stat. sol. (b) 223, 65 (2001)

[73] Takafumi Sato, Seigo Souma, Kosuke Nakayama, Kensei Terashima, Katsuaki Sugawara, Takashi Takahashi, Yoichi Kamihara, Masahiro Hirano, and Hideo Hosono, Superconducting Gap and Pseudogap in Iron-Based Layered Superconductor $\mathrm{La}\left(\mathrm{O}_{1-}\right.$ $\left.{ }_{x} F_{x}\right) F e A s$, J. Phys. Soc. Jpn. 77 (2008) 063708, available on arXiv:0805.3001v1 [cond-mat.supr-con].

[74] S. Takele and G.R. Hearne, Electrical transport, magnetism and spin-state configurations of high-pressure phases of FeS, Physical Review B 60. No. 7, p. 4401 (15 August 1999-I)

[75] G. Patermarakis, J. Papaioannou, H. Karayianni, and K. Masavetas, Interpretation of Electrical Conductance Transition of Hematite in the Spin-Flip Magnetic Transition Temperature Range, Journal of the Electrochemical Society 151 (8) J62-J68 (2004)

[76] J.E. McCrone, J.R. Cooper, J.W. Loram, and J.L. Tallon, Fielddependent anomalies in the thermodynamic and transport properties of $\mathrm{RuSr}_{2} \mathrm{GdCu}_{2} \mathrm{O}_{8}$ - evidence for a spin-flop transition, unpublished. 
[77] Naoshi Suzuki and Minoru Otani, Pressure-induced insulatormetal transition and collapse of magnetism in molecular solid oxygen, Polyhedron 20 (2001) 1381-1385

[78] Marvin L. Cohen, Superconductivity in Many-Valley Semiconductors and in Semimetals, Physical Review 134, No 2A (20 April 1964)

[79] J. Appel, Soft-Mode Superconductivity in $\mathrm{SrTiO}_{3-\mathrm{x}}$, Physical Review 180, no. 2 (10 April 1969)

[80] H.P.R. Frederikse, J.F. Schooley, W.R. Thurber, E Pfeiffer, and W.R. Hosler, Superconductivity in ceramic, mixed titanates. Physical Review Letters 16, No.13, 579-581 (28 March 1966)

[81] V.L. Gurevich, A.I. Larkin, and Yu. A. Firsov, On the possibility of superconductivity in semiconductors, Soviet Physics - Solid State 4, No. 1, pp 131-134 (July 1962)

[82] Marvin L. Cohen, Superconductivity in Low-Carrier-Density Systems: Degenerate Semiconductors, in Vol 1 Superconductivity Ed. by R. D. Parks, Marcel Dekker, Inc, New York 1969

[83] $\mathrm{H}$. Ehrenreich, Screening effects in polar semiconductors, in Advances in Semiconductor Science, J. Phys. Chem Solids, Pergamon Press 1959 Vol 8, pp130-135 (London)

[84] J. Appel, Soft-Mode Superconductivity in $\mathrm{SrTiO}_{3-x}$ and $\mathrm{Ca}_{y} \mathrm{Sr}_{1-\mathrm{y}} \mathrm{TiO}_{3-\mathrm{x}}$, Physica 55, 577-584 (October 1971) 
[85] H. Sato, T. Minami, S. Takata, T. Yamada, Transparentconducting p-type NiO thin films prepared by magnetron sputtering, Thin Solid Films. Vol. 236, no. 1-2, pp. 27-31. (1993)

[86] Toshio Kamiya and Hideo Hosono, Electronic Structures and Device Applications of Transparent Oxide Semiconductors: What is the Real Merit of Oxide Semiconductors?, International Journal of Applied Ceramic Technology Int. J. Appl. Ceram. Technol., 2 [4] 285-294 (2005)

[87] Su Sheng, Guojia Fang, Chun Li, Sheng Xu, and Xingzhong Zhao, p-type transparent conducting oxides, phys. stat. sol. (a) 203, No. 8, 1891-1900 (2006)

[88] Hidenori Hiramatsu, Kazushige Ueda, Toshio Kamiya, Hiromichi Ohta, Masahiro Hirano, and Hideo Hosono, Optical Properties and Two-Dimensional Electronic Structure in Wide-Gap Layered Oxychalcogenide: $\mathrm{La}_{2} \mathrm{CuO}_{2} \mathrm{Se}_{2}$, J. Phys. Chem. B 108, 17344-17451 (2004)

[89] G. E. Jellison, Jr., L. A. Boatner, D. H. Lowndes, R. A. McKee, and M. Godbole, Optical functions of transparent thin films of $\mathrm{SrTiO}_{3}$, $\mathrm{BaTiO}_{3}$, and $\mathrm{SiO}_{x}$ determined by spectroscopic ellipsometry, Applied Optics, Vol. 33, Issue 25, pp. 6053-6058 (September 1 1994)

[90] Interview with Prof. Hideo Hosono http://sciencewatch.com/dr/erf/2008/08decerf/08decerfHoso/ 
[91] R. S. Markiewicz, Pseudogaps in the Van Hove Jahn-Teller scenario, J. Phys. Chem. Solids, Vol. 56, No. 12, pp. 1637-1639 (1995)

[92] Quantum Design, Oxygen Contamination, MPMS Application Note 1014-210 03/04/1997

http://www.qdusa.com/resources/pdf/mpmsappnotes/1014-210.pdf

[93] Hai-Hu Wen, Superconducting gap on the Fermi arcs and condensation energy in $\mathrm{La}_{2-x} \mathrm{Sr}_{x} \mathrm{CuO}_{4}$ single crystals , Journal of Physics and Chemistry of Solids, 69, Iss 12, pp 3236-3239 (December 2008)

[94] Gregor Witte, Probing metallization transitions in twodimensional alkali metal films by metastable He-atom scattering, J. Phys.: Condens. Matter 16, S2937-2952, (2004)

[95] K.F. Herzfeld, On atomic properties which make an element a metal, Physical Review 29, Iss. 5 (May, 1927)

[96] P.P. Edwards, N.F. Mott and A.S. Alexandrov, The InsulatorSuperconductor Transformation in Cuprates, Journal of Superconductivity 11, No. 1 (1998)

[97] Robert A. Bari, Superconductivity, Ferroelectricity, and the Mott Insulator, Physical Review B 7, No. 8, 2128-2132 (1 March 1973) 
[98] S.-L. Drechsler, H. Rosner, M. Grobosch, G. Behr, F. Roth, G. Fuchs, K. Koepernik, R. Schuster, J. Malek, S. Elgazzar, M. Rotter, D. Johrendt, H-H. Klauss, B. Büchner, M. Knupfer, New insight into the physics of iron pnictides from optical and penetration depth data, arXiv:0904.0827v1 [cond-mat.supr-con] (April 2009)

[99] Jan Zaanen, Sudip Chakravarty, T. Senthil, Philip Anderson, Patrick Lee, Jörg Schmalian, Masatoshi Imada, David Pines, Mohit Randeria, Chandra Varma, Matthias Vojta, and Maurice Rice, Towards a complete theory of high $T_{c}$, Nature Physics, 2, 138-143, (March 2006)

[100] B. Vilquin, T. Kanki, T. Yanagida, H. Tanaka and T. Kawai, Effect of Sr doping on $\mathrm{LaTiO}_{3}$ thin films, Applied Surface Science 244, Iss 1-4, pp 494-497 (15 May 2005)

$12^{\text {th }}$ International Conference on Solid Films and Surfaces

[101] K. H. Kim, D. P. Norton, J. D. Budai, M. F. Chisholm, B. C. Sales, D. K. Christen, C. Cantoni, Epitaxial structure and transport in $\mathrm{LaTiO}_{3+x}$ films on (001) $\mathrm{SrTiO}_{3}$, physica status solidi (a) 200, Issue 2 , pp $346-351$ (2003)

[102] D. B. McWhan, T. M. Rice, and J. P. Remeika, Mott Transition in Cr-Doped $\mathrm{V}_{2} \mathrm{O}_{3}$, Phys. Rev. Lett. 23, 1384 - 1387 (1969)

[103] J. M. Honig and J. Spałek, Metal-insulator phase transitions and related critical phenomena in $\mathrm{NiS}_{2-\mathrm{x}} \mathrm{Se}_{x}$, Current Opinion in Solid State and Materials Science 5, Issue 4, pp 269-279 (August 2001) 
[104] P.W. Anderson, Superconductivity in the past and the future, in Vol 2 Superconductivity Ed. by R. D. Parks, Marcel Dekker, Inc, New York 1969

[105] A. Lanzara, P.V. Bogdanov, X.J. Zhou, S.A. Kellar, D.L. Feng, E.D. Lu, T. Yoshida, H. Eisaki, A. Fujimori, K Kishio, J.-I.

Shimoyama, T. Noda, S. Uchida, Z. Hussain \& Z.-X. Shen, Evidence for ubiquitous strong electron-phonon coupling in high-temperature superconductors, Nature 412, p. 510 (2 August 2001)

[106] K. Motida and K. Suzuki, Evidence for strong electron-phonon coupling in double-layered cuprate superconductors, Physica B: Condensed Matter 263-264, pp. $772-774$ (March 1999)

[107] C. Gadermaier, A. S. Alexandrov, V. V. Kabanov, P. Kusar, T. Mertelj, X. Yao, C. Manzoni, G. Cerullo, D. Mihailovic, Electronphonon coupling in cuprate high-temperature superconductors determined from exact electron relaxation rates, arXiv:0902.1636v2 [cond-mat.supr-con] (February 2009)

[108] http://www.ncnr.nist.gov/staff/jeff/Multiferroics.html There are many formal papers on specific aspects multiferroics, but it is a rather new field and details have not yet reached the textbooks. This site of Prof. J.W. Lynn of NIST and the University of Maryland covers the basics of the general physics involved. 
